\title{
An $r$-Matrix Approach to Nonstandard Classes of Integrable Equations
}

\author{
By
}

Boris Konopelchenko* and Walter Oevel**

\begin{abstract}
Three different decompositions of the algebra of pseudo-differential operators and the corresponding $r$-matrices are considered. Three associated classes of nonlinear integrable equations in $1+1$ and $2+1$ dimensions are discussed within the framework of generalized Lax equations and Sato's approach. The 2+1-dimensional hierarchies are associated with the Kadomtsev-Petviashvili (KP) equation, the modified KP equation and a Dym equation, respectively. Reductions of the general hierarchies lead to other known integrable $2+1$ dimensional equations as well as to a variety of integrable equations in $1+1$ dimensions. It is shown, how the multi-Hamiltonian structure of the 1+1-dimensional equations can be obtained from the underlying $r$-matrices. Further, intimate relations between the equations associated with the three different $r$-matrices are revealed. The three classes are related by Darboux theorems originating from gauge transformations and reciprocal links of the Lax operators. These connections are discussed on a general level, leading to a unified picture on (reciprocal) Bäcklund and auto-Bäcklund transformations for large classes of integrable equations covered by the KP, the modified KP, and the Dym hierarchies.
\end{abstract}

\$1 Introduction

$\S 2$ General Background and Basic Definitions

$\S 3$ Three Classes of Integrable Equations in $1+1$ Dimensions

\section{$\S 3.1$ Reductions}

\$3.2 A List of Integrable 1+1-Dimensional Equations

$\S 3.3$ A Discrete Invariance and Kupershmidt Reductions

$\S 3.4$ The Hamiltonian Concept

$\S 4$ Sato Approach: Three Classes of Integrable Equations in $2+1$ Dimensions

$\S 4.1$ The KP Hierarchy and Nonstandard Sato Equations

Communicated by M. Kashiwara, September 9, 1991.

1991 Mathematics Subject Classifications: 58 F07

* Institute of Nuclear Physics, Novosibirsk-90, 630090, Russia

** Department of Mathematical Sciences, Loughborough University of Technology, LE11 3TU, United Kingdom 


\section{$\S 4.2$ Kupershmidt Reductions}

$\S 5$ Gauge Transformations and Reciprocal Links: Darboux Theorems

$\S 6$ Conclusions

References

\section{$\S 1$. Introduction}

Large classes of nonlinear partial differential equations are integrable by the inverse spectral transform (IST) method and its modifications (see e.g. Refs. [1-4]). There exist different approaches to a unified description of these integrable equations and their properties. Two of them reveal a deep interrelation between the integrable equations and infinite-dimensional algebras. One is based on the treatment of partial differential equations as dynamical systems on the infinite dimensional algebra of pseudo-differential operators (see e.g. Refs. [5-9]). Introducing an infinite set of "time" variables one can also treat the integrable equations as flows on infinite dimensional Grassmannian manifolds ([10-14]). This method (Sato and $\tau$-function approach) yields a beautiful and adequate description for a class of $2+1$-dimensional integrable equations.

Another approach to a unified description of the integrable equations is based on the use of a simple and powerful algebraic tool, the so-called $r$-matrix ([15-17]). This approach can be formulated in a rather abstract algebraic way and gives a simple but effective method for the analysis of the Hamiltonian structures of the integrable systems.

The Hamiltonian construction of the integrable equations using pseudo-differential operators becomes quite transparent when using the terminology of $r$-matrices. Based on results by Gelfand and Dikii ([5]) Adler ([7]) used a Lie algebraic setting to describe integrable partial differential equations such as the Korteweg-de Vries (KdV) equation via their Lax representations. As an important consequence it turned out that integrable systems of different nature (discrete lattices systems or differential equations) may be constructed in a similar manner using Lie algebraic techniques. The celebrated Adler-Kostant-Symes (AKS) scheme starts with a (dual) Lie algebra as the natural phase space for the integrable equations. The Lie-Poisson bracket associated with the Lie algebra structure provides a natural Hamiltonian structure, the invariant functions provide a natural set of functions in involution on the algebra. In order to obtain a nontrivial integrable dynamics for these functions only a few additional structures have 
to be provided, which are again of purely Lie algebraic nature. As simplest example, a decomposition of the original algebra into proper subalgebras gives rise to a hierarchy of integrable Hamiltonian equations. It turned out that this construction may be regarded as a special case of a yet larger picture. Following Drinfeld's ideas ([15]) Semenov showed that the notion of classical $r$-matrices leads to an algebraic construction of integrable systems generalizing the AKS scheme. Not only a systematic view of the multiHamiltonian nature of such equations can be given in terms of $r$-matrices ([16]), they also provide a general interpretation of the dressing transformations used to solve these equations in terms of group factorizations ([17]). In Refs. $[18,19]$ it was shown that - subject to suitable technical assumptionsthere are in fact three natural Poisson brackets associated with such classical $r$-matrices. They lead to an abstract tri-Hamiltonian formulation of the Lax equations describing the nonlinear integrable systems. Applications of the general construction to the particular algebra of pseudo-differential operators used by Adler ([7]) were given in Ref. [19], leading to a compact formulation of the multi-Hamiltonian structures found for certain classes of integrable hierarchies.

In this paper we again pick up this construction on the algebra $g$ of pseudo-differential symbols. Considering Lax operators $L=\Sigma_{i} u_{i}(x) \partial^{i}$ it turns out that there are in fact three natural and simple $r$-matrices on this algebra. They originate from decompositions $g=g_{\geq k} \oplus g_{<k}$ into subalgebras of the form

$$
g_{\geq k}=\left\{\sum_{i \geq k} u_{i} \partial^{i}\right\}, \quad g_{<k}=\left\{\sum_{i<k} u_{i} \partial^{i}\right\}, \quad k=0,1,2
$$

The three given choices for $k$ are distinguished by the fact that both of the complementary subspaces define proper Lie subalgebras. Application of the general scheme with these particular $r$-matrices results in the following generalized Lax representation of integrable equations

$$
\frac{d}{d t_{q}} L=\left[P_{\geq k}\left(L^{q}\right), L\right],
$$

where $k=0,1,2$ and $q$ may assume suitable integer or rational values. By $P_{\geq k}$ we denote the projection to the subalgebra $g_{\geq k}$. Restriction to the invariant subspace of purely differential operators for $k=0$ yields the 
well-known ("standard") Gelfand-Dikii hierarchy of equations, which includes the KdV-hierarchy as a particular case. Restriction to another invariant subspace of operators of the particular form $L=\partial+\Sigma_{i<0} u_{i}(x) \partial^{i}$ with $k=0$ leads to Sato's approach to the integrable Kadomtsev-Petviashvili (KP) hierarchy.

The generalized Lax-equation (1.2) for $k \neq 0$ were considered by Reiman ([20]) and Kupershmidt ([21]), who called them the "nonstandard cases". He demonstrated that the hierarchies of the Kaup-Broer system and the modified $\mathrm{KdV}$ are related to the first nonstandard case $k=1$. He analysed the Hamiltonian structure of theses hierarchies and other properties. He also showed that some of the equations in (1.2) admit a reduction to the invariant subspace on which the adjoint $L^{\dagger}$ of the Lax operator is related to $L$ by $L^{\dagger}=(-1)^{N} \partial^{k} L \partial^{-k}$. Here the sign is determined by the order $N$ of $L$. We will refer to this reduction as the Kupershmidt reduction.

For all three classes $k=0,1,2$ we will discuss in detail subspaces of Lax operators which are left invariant by the dynamics (1.2). A list of the simplest equations connected with (1.2) will be given. Regarding the KdV and the modified $\mathrm{KdV}$ equations as the prototype equations associated with the classes $k=0$ and $k=1$, respectively, one discovers the Dym equation as the most prominent example of the last class $k=2$. Following Sato's approach hierarchies of integrable $2+1$-dimensional equations can be extracted from (1.2). In this context the nonstandard cases were also observed recently by Kiso ([22]). The class $k=0$ is associated with the KP hierarchy, whereas the nonstandard cases $k=1$ and $k=2$ lead to the modified KP and a 2+1-dimensional Dym equation found in Ref. [23]. A description of the three basic 2+1-dimensional hierarchies and their links is given in [24]. In this paper we will concentrate on reduction aspects of these hierarchies, that is, the emphasis will be placed on realizations in terms of 1+1-dimensional nonlinear equations.

In Section 2 we review the background on $r$-matrices used in the Hamiltonian constructions following later on. We also discuss the prototype equations $\mathrm{KdV}$, modified $\mathrm{KdV}$ and Dym in terms of fractional powers of Lax operators. Section 3 is devoted to realizations of the general Lax equation (1.2) in terms of 1+1-dimensional equations. In Section 3.1 the reduction properties of (1.2) are discussed in detail. A characterization of admissible classes of Lax operators is given, for which the Lax equations lead to a consistent dynamics for the fields parametrizing the Lax operators. In Section 3.2 a list of the simplest isospectral equations is given 
for operators up to fifth order. It turns out that a surprising variety of both well-known and novel integrable equations are covered by the Lax equations (1.2). Additional reductions given by the Kupershmidt constraints are discussed in Section 3.3. The Hamiltonian background of the $r$-matrix approach is used in Section 3.4 to derive the Poisson brackets for the nonlinear equations given by (1.2). It turns out that the three abstract Poisson structures associated with $r$-matrices can indeed be used to extract the multi-Hamiltonian formulations for these equations, when suitable reduction techniques are used. The curious role of the general quadratic bracket associated with the cases $k=1$ and $k=2$ will be pointed out. In Section 4 realizations of (1.2) in terms of $2+1$-dimensional equations are discussed. In Section 4.1 the three general hierarchies are identified as the hierarchies of the $\mathrm{KP}$, the modified $\mathrm{KP}$ and the 2+1-dimensional Dym equations, respectively. In Section 4.1 the Kupershmidt constraints are considered. The resulting nonlinear equations are shown to be $2+1$ dimensional versions of the 5 th order $\mathrm{KdV}$-type equation of Kupershmidt ([25]), of the Sawada-Kotera equation ([26]) (the "BKP" in the notation of Ref. [12]) and a higher order Dym equation first constructed by Konopelchenko and Dubrovsky ([23]). Section 5 is devoted to a general discussion of (reciprocal) Bäcklund and auto-Bäcklund transformations. Gauge transformations of the Lax operators and reciprocal links (transformations of the independent variables) provide a surprisingly general and unified picture of the relations between the three classes (1.2). A large number of Darboux-type transformations is discussed, leading to a coherent concept for a variety of both well-known and novel transformations between the integrable hierarchies.

\section{$\S 2$. General Background and Basic Definitions}

The construction of the integrable nonlinear equations discussed here is based on the ideas of classical $r$-matrices as discussed by Semenov ([16]). As we will apply these ideas to one particular algebra (of pseudodifferential symbols), we will not give an account of these notions in full generality, but review the relevant structures in a slightly restricted form suitable for the applications following later on. Having in mind Lax equations we start with an abstract associative algebra $g$ of Lax operators as the natural phase space of integrable dynamical systems. We assume that $g$ bears a symmetric, non-degenerate trace form $t r: g \rightarrow \boldsymbol{R}$, so that $g$ can be identified 
with its dual $g^{*}$ via the symmetric pairing

$$
<a, b>=\operatorname{tr}(a b)=\operatorname{tr}(b a)=<b, a>, \quad a, b \in g .
$$

Considering the natural Lie algebra structure $[a, b]=a b-b a$ on $g$, we thus have an invariant metric satisfying

$$
<a,[b, c]\rangle=<c,[a, b]>
$$

for arbitrary $a, b, c \in g$. A classical $r$-matrix on $g$ is a linear map $r: g \rightarrow g$, so that the modified bracket

$$
[a, b]_{r}=[r(a), b]+[a, r(b)]
$$

satisfies the Jacobi-identity and hence provides a second Lie algebra structure on $g$. As shown in Ref. [16], a sufficient condition for a linear map $r$ to be an $r$-matrix is given by the so-called modified Yang-Baxter equation

$$
[r(a), r(b)]+[a, b]=r\left([a, b]_{r}\right)
$$

Complete classifications of such $r$-matrices have been given for certain types or Lie-algebras $([27,28])$, for our purposes only the following simple type of $r$-matrices will be relevant. We assume that the Lie algebra $g$ can be decomposed into two smaller Lie algebras $g_{+}$and $g_{-}$, i.e.

$$
g=g_{+} \oplus g_{-}, \quad\left[g_{ \pm}, g_{ \pm}\right] \subset g_{ \pm} .
$$

Denoting the projections onto these subalgebras by $P_{ \pm}$, it is easy to verify that

$$
r=P_{+}-P_{-}=\left\{\begin{array}{rll}
1 & \text { on } & g_{+} \\
-1 & \text { on } & g_{-}
\end{array}\right.
$$

satisfies the Yang-Baxter equation (2.4) and hence constitutes an $r$-matrix on $g$. Here 1 is to be the identity map. The corresponding bracket (2.3) is calculated as $[a, b]_{r}=2\left[P_{+}(a), P_{+}(b)\right]-2\left[P_{-}(a), P_{-}(b)\right]$, which clearly is a Lie bracket as $g_{ \pm}$were supposed to be Lie subalgebras of $g$. The new Lie product endows $g=g^{*}$ with a natural Poisson structure, that is, the Lie Poisson bracket 


$$
\{F, H\}_{1}(L)=<L,[\nabla F(L), \nabla H(L)]_{r}>
$$

where $L \in g$ and $F, H$ are functions on $g$. In fact, three potential Poisson brackets are introduced with the skew symmetric tensors $\mathscr{P}_{i}(L): g \rightarrow g$ defined by

$$
\begin{aligned}
& \mathscr{P}_{1}(L) \nabla H=[r(\nabla H), L]+r^{*}([\nabla H, L]), \\
& \mathscr{P}_{2}(L) \nabla H=[r(L \nabla H+\nabla H L), L]+L r^{*}([\nabla H, L])+r^{*}([\nabla H, L]) L, \\
& \mathscr{P}_{3}(L) \nabla H=[r(L \nabla H L), L]+L r^{*}([\nabla H, L]) L,
\end{aligned}
$$

where $r^{*}$ is the adjoint of $r$ relative to the trace duality (2.1), that is $\langle r(a), b\rangle=\left\langle a, r^{*}(b)\right\rangle$. These tensors shall be called "linear", "quadratic" and "cubic", referring to their polynomial dependence on the point $L \in g$. Among the corresponding brackets

$$
\{F, H\}_{i}(L)=\left\langle\nabla F, \mathscr{P}_{i}(L) \nabla H>, i=1,2,3,\right.
$$

the linear bracket coincides with the Lie Poisson structure (2.7) and hence defines a Poisson bracket for arbitrary $r$-matrices. It was shown in Refs. $[18,19]$ that also the cubic bracket will be a Poisson bracket, automatically, for all $r$-matrices satisfying the Yang-Baxter equation (2.4). The Poisson properties of the quadratic bracket are more delicate: equation (2.4) for $r$ is not sufficient to guarantee that $\{., .\}_{2}$ will be a Poisson bracket. Additional assumptions on $r$ have to be imposed. According to Refs. [18,19] a sufficient condition for the quadratic bracket to be a Poisson bracket is given, if $r$ and its skew-adjoint part $\tilde{r}=\frac{1}{2}\left(r-r^{*}\right)$ both satisfy (2.4). In particular, for skew-adjoint solutions $r^{*}=-r$ of the Yang-Baxter equation (2.4), one finds a set of three Poisson brackets $(2.8) /(2.9)$ associated with the $r$-matrix. We remark that all three brackets are related by the simple deformation

$$
\begin{aligned}
& \mathscr{P}_{2}(L+\varepsilon 1)=\mathscr{P}_{2}(L)+2 \varepsilon \mathscr{P}_{1}(L), \\
& \mathscr{P}_{3}(L+\varepsilon 1)=\mathscr{P}_{3}(L)+\varepsilon \mathscr{P}_{2}(L)+\varepsilon^{2} \mathscr{P}_{1}(L),
\end{aligned}
$$

where 1 is the identity element of the algebra. It is well known in the theory of soliton equations that an important property of multi-Hamiltonian formulations for such equations is the compatibility of the Poisson tensors ([29]). We recall that two Poisson brackets are called compatible, if their sum is again a Poisson bracket. From (2.10) it is readily seen that all three 
brackets will be compatible, automatically, if $r$ is such that all three brackets are indeed Poisson brackets. In particular, for any solution $r$ of the Yang-Baxter equation (2.4), the linear and the cubic bracket are Poisson structures. Using (2.10.ii) it is easy to see that these two brackets are compatible if and only if also the quadratic bracket is a Poisson structure.

The introduction of an $r$-matrix on $g$ thus turns the algebra into a Hamiltonian phase space. Further, it turns out that there is a natural set of functions in involution relative to all three brackets discussed above. These are the so-called Casimir functions on $g^{*}$, which are invariant with respect to the co-adjoint action of the Lie group generated by $g$. Infinitesimally, they are characterized by the fact that their gradients, evaluated at a point $L \in g=g^{*}$, commute with $L$. As we have assumed a non-degenerate trace form $t r$ on $g$, we will consider the Casimir functions given by the traces of powers of $L$. Their gradients relative to the trace duality are given as powers of $L$, that is, we will be interested in

$$
C_{q}(L)=\frac{1}{q} \operatorname{tr}\left(L^{q}\right), \quad \nabla C_{q}(L)=L^{q-1}
$$

The involutivity of these functions with respect to the three brackets given by (2.8) is evident. The Hamiltonian equations associated to these Casimir functions are given by $\frac{d}{d t} L=\mathscr{P}_{i} \nabla C_{q}$. Observing the particular form of the tensors (2.8) one finds the following hierarchy of equations

$$
\frac{d}{d t_{q}} L=\left[r\left(L^{q}\right), L\right]=P_{1} \nabla C_{q+1}=\frac{1}{2} P_{2} \nabla C_{q}=P_{3} \nabla C_{q-1}
$$

which are evidently tri-Hamiltonian with respect to the three brackets above (provided $r$ is such that all three brackets are indeed Poisson brackets). In the Lax equations (2.12) we have introduced an evolution parameter ("time") $t_{q}$ for each power $q$. For any $r$-matrix each two evolution equations in the hierarchy (2.12) commute due to the involutivity of the Casimir functions $C_{q}$. Each equation admits all the Casimir functions as a set of conserved quantities in involution. In this sense we will regard (2.12) as a hierarchy of integrable evolution equations. In this construction we have defined the equation based on a Hamiltonian framework involving the $r$-matrix. The Lax form (2.12) owes its simplicity to the assumption of the trace-duality 
(2.1) on the algebra $g$. We remark, however, that for the construction of a hierarchy of commuting equations of the form $\frac{d}{d t} L=\left[r\left(\nabla C_{q}\right), L\right]$ neither the Hamiltonian framework nor special assumptions on the duality are required. If we consider two evolution equations

$$
\frac{d}{d t} L=[r(A), L], \quad \frac{d}{d t} L=[r(\tilde{A}), L]
$$

then the identity

$$
\frac{d}{d t} \frac{d}{d t} L-\frac{d}{d t} \frac{d}{d t} L=\left[r\left([A, \tilde{A}]_{r}\right)-[r(A), r(\tilde{A})], L\right]
$$

immediately shows that the two equations (2.13) will commute, if $r$ satisfies the modified Yang-Baxter equation and the expressions $A=A(L)$ and $\tilde{A}=\tilde{A}(L)$ commute with $L$. Hence, the hierarchy of evolution equations (2.12) will commute independent of its Hamiltonian background, that is, independent of the presence of a trace formalism.

For the special $r$-matrices (2.6) originating from a Lie algebra decomposition of the algebra $g$, we can use the identity

$$
0=\left[L^{q}, L\right]=\left[P_{+}\left(L^{q}\right)+P_{-}\left(L^{q}\right), L\right] \rightarrow\left[P_{-}\left(L^{q}\right), L\right]=-\left[P_{+}\left(L^{q}\right), L\right]
$$

to rewrite the integrable hierarchy as

$$
\frac{d}{d t_{q}} L=\left[r\left(L^{q}\right), L\right]=\left[P_{+}\left(L^{q}\right)-P_{-}\left(L^{q}\right), L\right]=2\left[P_{+}\left(L^{q}\right), L\right]=-2\left[P_{-}\left(L^{q}\right), L\right]
$$

Here we remark that the commutativity of these equations for different values of $q$ is also reflected in the following compatibility equations for the projected powers $P_{+}\left(L^{q}\right)$. Using the fact that $g_{ \pm}$form subalgebras in $g$, it is readily verified that they satisfy the zero-curvature equations

$$
\frac{d}{d t_{q}}\left(P_{+}\left(L^{\tilde{q}}\right)\right)-\frac{d}{d t_{\tilde{q}}}\left(P_{+}\left(L^{q}\right)\right)+\left[P_{+}\left(L^{\tilde{q}}\right), P_{+}\left(L^{q}\right)\right]=0
$$

We will apply this formalism to the algebra of pseudo-differential symbols 
as discussed by Adler ([7]). We will consider the operator algebra

$$
g=\left\{\sum_{-\infty<i \ll \infty} u_{i}(x) \partial^{i}\right\}
$$

where $\partial=\partial / \partial_{x}$ and the coefficients $u_{i}$ are to be functions of the "space variable" $x$. The negative powers of $\partial$ are to be understood in the following way. One introduces a formal integration symbol $\partial^{-1}$, for which algebraic multiplication with a multiplication operator $a$ (given by a function $a=a(x)$ ) is defined as the formal series

$$
\partial^{-1} a=a \partial^{-1}-a_{x} \partial^{-2}+a_{x x} \partial^{-3}-\cdots
$$

In fact, this algebraic rule is just a special case of the general Leibniz rule

$$
\partial^{i} a=\sum_{l \geq 0}\left(\begin{array}{l}
i \\
l
\end{array}\right) \frac{\partial^{l} a}{\partial x^{l}} \partial^{i-l}, \quad\left(\begin{array}{l}
i \\
l
\end{array}\right)=\frac{i(i-1) \cdots(i-l+1)}{1 \times 2 \times \cdots \times l}
$$

for differential operators, now extended to negative powers $i$. With these assumptions we have fixed the algebraic structure of the set (2.18), which corresponds to the multiplication used by Adler. The decisive observation in Ref. [7] was that the trace form

$$
\operatorname{tr}\left(\sum_{i} u_{i}(x) \partial^{i}\right)=\int u_{-1}(x) d x
$$

yields a symmetric and non-degenerate pairing on $g$. In (2.21) the integration denotes the equivalence class of differential expressions modulo total derivatives. As a consequence, for operators $L=\Sigma u_{i} \partial^{i}$, vectorfields $\frac{d}{d t} L$ and gradients $\nabla H$ are conveniently parametrized by

$$
\frac{d}{d t} L=\sum_{i} u_{i t} \partial^{i}, \quad \nabla H=\sum_{i} \partial^{-1-i} \frac{\delta H}{\delta u_{i}}
$$

where

$$
\frac{\delta H}{\delta u_{i}}=\frac{\partial H}{\partial u_{i}}-\left(\frac{\partial H}{\partial u_{i x}}\right)_{x}+\left(\frac{\partial H}{\partial u_{i x x}}\right)_{x x}-\cdots
$$


is the usual variational derivative of a functional $H=H(L)=H\left(u_{i}, u_{i x}, ..\right)$ in terms of the Euler operator. In these frames the trace duality assumes the usual Euclidean form

$$
<\nabla H, \frac{d}{d t} L>=<\frac{d}{d t} L, \nabla H>=\sum_{i} \int \frac{\delta H}{\delta u_{i}} u_{i t} d x
$$

The integrable equations considered in Ref. [7] are given by the $r$-matrix (2.6) associated with the Lie algebra decomposition $g=g_{+} \oplus g_{-}$with

$$
g_{+}=g_{\geq 0}=\left\{\sum_{i \geq 0} u_{i} \partial^{i}\right\}, \quad g_{-}=g_{<0}=\left\{\sum_{i<0} u_{i} \partial^{i}\right\}
$$

that is, operators are split into purely differential parts versus integration parts. The corresponding integrable nonlinear systems are given by the Lax equations (2.16), that is, $\frac{d}{d t_{q}} L=2\left[P_{\geq 0}\left(L^{q}\right), L\right]$, where $P_{\geq 0}\left(\Sigma_{i} a_{i} \partial^{i}\right)=\Sigma_{i \geq 0} a_{i} \partial^{i}$ represents the projection to the purely differential part of the operator. These are the Lax equations originally studied by Gelfand and Dikii ([5]).

In Ref. [21] Kupershmidt observed that also modifications of these Lax equations will lead to integrable systems. He considered Lax equations of the type

$$
\frac{d}{d t_{q}} L=\left[P_{\geq k}\left(L^{q}\right), L\right], \quad k=0,1,2,
$$

where $P_{\geq k}\left(\Sigma_{i} a_{i} \partial^{i}\right)=\Sigma_{i \geq k} a_{i} \partial^{i}$. The additional cases $k=1$ and $k=2$ were titled the "nonstandard" cases in Ref. [21]. In the framework of $r$-matrices and Lie-algebra decompositions this construction can be understood easily. We consider simple decompositions of $g$ of the form

$$
g=g_{+} \oplus g_{-}=g_{\geq k} \oplus g_{<k}, \quad g_{\geq k}=\left\{\sum_{i \geq k} u_{i} \partial^{i}\right\}, \quad g_{<k}=\left\{\sum_{i<k} u_{i} \partial^{i}\right\}
$$

With $P_{+}=P_{\geq k}$ and $P_{-}=P_{<k}$ we denote the projections to these subspaces. In order to ensure that the maps $r_{k}=P_{\geq k}-P_{<k}$ define classical $r$-matrices, we have to look for those cases of $k$, for which both $g_{\geq k}$ and $g_{<k}$ are Lie subalgebras of $g$. It is readily verified that $g_{\geq k}$ constitutes a closed subalgebra 
of differential operators for any integer values $k \geq 0$. On the other hand, due to $(2.19) /(2.20)$, it is also easily checked that $g_{<k}$ constitutes a closed subalgebra of integro-differential symbols for any integer values $k<2$. The common cases $k=0,1$ and 2 correspond precisely to the three classes of integrable equations considered by Kupershmidt.

For the rest of the paper, we restrict $k$ to one of the three choices $k=0,1$ or 2 and discuss the properties of the three Lax hierarchies (2.16)

$$
\frac{d}{d t_{q}} L=\left[P_{\geq k}\left(L^{q}\right), L\right]=-\left[P_{<k}\left(L^{q}\right), L\right], \quad k=0,1,2
$$

where the irrelevant factor 2 in $(2.16)$ will be dropped for convenience in the rest of the paper.

The main advantage of having formulated Kupershmidt's equations (2.26) in terms of classical $r$-matrices is the fact that the Hamiltonian nature of the nonlinear integrable systems encoded in these Lax equations is built right into their construction. In particular, Kupershmidt revealed three local Hamiltonian formulations for a system of classical dispersiveless long wave equations, which turns out to be one of the realizations of the equations (2.28) for $k=1$ (see also Section 3.2). As we have available three candidates (2.8) for Poisson tensors one expects that these abstract brackets reduce to the specific Hamiltonian structures found by Kupershmidt upon insertion of a specific Lax operator $L$ corresponding to these equations. This, however, turns out to be the case only in a restricted way. Only for the $k=0$ class of (2.28) all three brackets are Poisson structures, so that the abstract multi-Hamiltonian structure (2.12) does indeed account for the specific multiHamiltonian structures and recursion operators associated with the corresponding nonlinear equations. For the other two cases $k=1$ and $k=2$ only the linear and the cubic bracket are Poisson structures for general $L$ in $g$. Thus, we have a general formalism to derive Hamiltonian formulations from two of the tensors (2.8). The technical assumptions needed to render the quadratic bracket a Poisson bracket are satisfied only for the $k=0$ case. Hence, from a general point of view, no further Hamiltonian formulations should be expected for the nonlinear equations encoded in the hierarchies associated with the $k=1$ and $k=2$ cases. As a surprising fact, though, the quadratic bracket will become a Poisson structure when imposing Dirac constraints to certain subclasses of operators. It will be demonstrated, that certain Hamiltonian formulations can be extracted from the quadratic 
bracket for the cases $k=1$ and $k=2$, too. This will be discussed in Section 3.4.

Before entering a systematic discussion of the integrable nonlinear systems hidden in (2.28), we look at the three simplest examples of equations related to the three choices for $k$ in (2.28). We consider the operators

$$
\begin{array}{ll}
\underline{k=0:} & L_{K d V}=\partial^{2}+u, \\
\underline{k=1:} & L_{m K d V}=\partial^{2}+2 v \partial+\lambda, \\
\underline{k=2:} & L_{D y m}=w^{2} \partial^{2}+\lambda_{1} x \partial+\lambda_{2},
\end{array}
$$

and try to evaluate the hierarchies of Lax equations (2.28) with these operators and the corresponding values for $k$ as indicated above. Here, $u, v$ and $w$ are the dynamical fields satisfying the nonlinear equations given by the Lax representation. For reasons to become obvious in the final Section 5 we have included arbitrary constant parameters $\lambda, \lambda_{1,2}$ in these operators. The subscripts are motivated by the fact that these operators constitute the well-known Lax operators associated to the $\mathrm{KdV}$ equation, the modified $\mathrm{KdV}$ equation and the Dym equation, respectively. The crucial point about the following analysis is that in all cases the isospectral hierarchy of equations associated with a given operator $L$ can be calculated from $L$ in a straightforward way. The recipe to obtain the second operator $P_{\geq k}\left(L^{q}\right)$ needed for the dynamical equations (2.28) is simply given by choosing a power $q$ of $L$ and applying one of the projections $P_{\geq k}$ to this operator. It should be noted, however, that integer powers $q$ of $L$ will not lead to any interesting dynamics, when we start with purely differential Lax operators. In the case $k=0$, for instance, we would have $P_{\geq 0}\left(L^{q}\right)=L^{q}$ for integer $q$, leading to the trivial dynamics $\frac{d}{d t_{q}} L=\left[L^{q}, L\right]=0$. As shown by Gelfand and Dikii ([5]) fractional powers of the differential operators will lead to interesting results. In context of the algebra (2.18) the relevant procedure can be summarized as follows. We first consider the Schrödinger operator $L_{K d V}$ of (2.29) and review how fractional powers can be calculated in a purely algebraic way. Using the formal integration symbol $\partial^{-1}$ we consider operators of the form

$$
L_{K d V}^{1 / 2}=\partial+a_{0}+a_{1} \partial^{-1}+a_{2} \partial^{-2}+a_{3} \partial^{-3}+\cdots
$$

Trying to turn the ansatz (2.30) into a formal square root of the Schrödinger operator, we identify the coefficients $a_{0}, a_{1}, a_{2}, \cdots$ by calculating 


$$
\begin{aligned}
L_{K d V}^{1 / 2} L_{K d V}^{1 / 2}= & \partial^{2}+2 a_{0} \partial+\left(a_{0 x}+a_{0}^{2}+2 a_{1}\right) \\
& +\left(a_{1 x}+2 a_{0} a_{1}+2 a_{2}\right) \partial^{-1}+(. .) \partial^{-2}+. .
\end{aligned}
$$

using (2.20) and $\partial \partial^{-1}=\partial^{-1} \partial=1$. Hence, requiring $\left(L_{K d V}^{1 / 2}\right)^{2}=\partial^{2}+u$, we can calculate all the coefficients $a_{0}, a_{1}, a_{2}, \ldots$, recursively in terms of the field $u$. In particular, one finds the formal expansion

$$
L_{K d V}^{1 / 2}=\partial+\frac{1}{2} u \partial^{-1}-\frac{1}{4} u_{x} \partial^{-2}+\frac{1}{8}\left(u_{x x}-u^{2}\right) \partial^{-3}+(. .) \partial^{-4}+\cdots
$$

Calculating the third power of (2.32) (or multiplying with $\partial^{2}+u$ ) one finds the operator

$$
L_{K d V}^{3 / 2}=\partial^{3}+\frac{3}{2} u \partial+\frac{3}{4} u_{x}+(. .) \partial^{-1}+(. .) \partial^{-2}+\cdots,
$$

which - by construction - commutes with $\partial^{2}+u$. Applying the projection $P_{\geq 0}$ we have extracted the second operator of the Lax formulation of the $\mathrm{KdV}$ equation as the purely differential part of (2.33):

$$
\begin{aligned}
& L_{K d V}=\partial^{2}+u, P_{\geq 0}\left(L_{K d V}^{3 / 2}\right)=\partial^{3}+\frac{3}{2} u \partial+\frac{3}{4} u_{x}, \\
& \frac{d}{d t} L_{K d V}=\left[P_{\geq 0}\left(L_{K d V}^{3 / 2}\right), L_{K d V}\right] \leftrightarrow 4 u_{t}=u_{x x x}+6 u u_{x} .
\end{aligned}
$$

In fact, the entire hierarchy of higher $\mathrm{KdV}$ equations can be obtained in the same fashion. For instance, including further terms in the expansions (2.32) one can easily calculate $L_{K d V}^{5 / 2}$ and consider its differential part. This operator will supplement the Schrödinger operator to form a Lax pair for the 5 th order $\mathrm{KdV}$ flow $16 u_{t}=u_{x x x x x}+10 u u_{x x x}+20 u_{x} u_{x x}+30 u^{2} u_{x}$ commuting with the KdV.

In a similar way one can extract the second operators for the Lax pairs of the modified $\mathrm{KdV}$ and the Dym equation out of the given Lax operators $L_{m K d V}$ and $L_{D y m}$ in (2.29). Using the same ansatz (2.30) for $L_{m K d V}^{1 / 2}$ and a modified ansatz $L_{D y m}^{1 / 2}=w \partial+a_{0}+a_{1} \partial^{-1}+$. for the Dym case, one calculates

$$
L_{m K d V}^{1 / 2}=\partial+v+\frac{1}{2}\left(\lambda-v_{x}-v^{2}\right) \partial^{-1}+(. .) \partial^{-2}+. .
$$




$$
\begin{aligned}
L_{D y m}^{1 / 2}= & w \partial+\frac{1}{2}\left(\frac{\lambda_{1} x}{w}-w_{x}\right)+\frac{1}{8}\left(2 w_{x x}-\frac{w_{x}^{2}}{w}+\frac{4 \lambda_{1} x w_{x}}{w^{2}}+\right. \\
& \left.\frac{4 \lambda_{2}-2 \lambda_{1}}{w}-\frac{\lambda_{1}^{2} x^{2}}{w^{3}}\right) \partial^{-1}+(. .) \partial^{-2}+. .,
\end{aligned}
$$

and

$$
\begin{aligned}
& L_{m K d V}^{3 / 2}=\partial^{3}+3 v \partial^{2}+\frac{3}{2}\left(\lambda+v_{x}+v^{2}\right) \partial+(. .)+(. .) \partial^{-1}+. . \\
& L_{D y m}^{3 / 2}=w^{3} \partial^{3}+\frac{3}{2}\left(w^{2} w_{x}+\lambda_{1} x w\right) \partial^{2}+(. .) \partial+(. .)+(. .) \partial^{-1}+. . .
\end{aligned}
$$

Using the projections $P_{\geq k}$ with $k=1$ for the modified $\mathrm{KdV}$ and $k=2$ for the Dym equation one obtains the Lax pairs for these equations:

$$
\begin{gathered}
L_{m K d V}=\partial^{2}+2 v \partial+\lambda, P_{\geq 1}\left(L_{m K d V}^{3 / 2}\right)=\partial^{3}+3 v \partial^{2}+\frac{3}{2}\left(\lambda+v_{x}+v^{2}\right) \partial, \\
\frac{d}{d t} L_{m K d V}=\left[P_{\geq 1}\left(L_{m K d V}^{3 / 2}\right), L_{m K d V}\right] \leftrightarrow 4 v_{t}=v_{x x x}-6 v^{2} v_{x}+6 \lambda v_{x},
\end{gathered}
$$

and

$$
\begin{gathered}
L_{D y m}=w^{2} \partial^{2}+\lambda_{1} x \partial+\lambda_{2}, P_{\geq 2}\left(L_{D y m}^{3 / 2}\right)=w^{3} \partial^{3}+\frac{3}{2}\left(w^{2} w_{x}+\lambda_{1} x w\right) \partial^{2}, \\
\frac{d}{d t} L_{D y m}=\left[P_{\geq 2}\left(L_{D y m}^{3 / 2}\right), L_{D y m}\right] \leftrightarrow 4 w_{t}=w^{3} w_{x x x}+3 \lambda_{1}^{2} x\left(1-\frac{x w_{x}}{w}\right) .
\end{gathered}
$$

For the special choice $\lambda_{1}=0$ of the constant parameter we have the Dym equation in its standard form.

Having identified $\mathrm{KdV}$, modified $\mathrm{KdV}$ and Dym equation as the simplest realizations of the three classes $(2.28)$ by choosing second order differential operators for $L$, we note that there are well known relations between these equations. The link between $\mathrm{KdV}$ and modified $\mathrm{KdV}$ is given by the celebrated Miura transformation

$$
u=\lambda-v_{x}+v^{2} .
$$


We also briefly review the reciprocal link of $\mathrm{KdV} /$ modified $\mathrm{KdV}$ to the Dym equation. One way of describing this link originates from the Painlevè analysis of integrable PDE's as introduced by Weiss et al ([30]). For both the $\mathrm{KdV}$ and the modified $\mathrm{KdV}$ the singularity field $\Phi$ used in this analysis has to satisfy the nonlinear equation

$$
4 \frac{\Phi_{t}}{\Phi_{x}}=\{\Phi ; x\}+\mu
$$

where $\{\Phi ; x\}=\left(\Phi_{x x} / \Phi_{x}\right)_{x}-\left(\Phi_{x x} / \Phi_{x}\right)^{2} / 2$ is the Schwarzian derivative and $\mu$ is an arbitrary constant parameter. In Ref. [31] it was noted that one may introduce the new independent variables $x^{\prime}=\Phi(x, t)$ and $t^{\prime}=t$. Then $w=\Phi_{x}$, expressed in terms of $x^{\prime}$ and $t^{\prime}$, will satisfy the Dym equation $4 w_{t^{\prime}}=w^{3} w_{x \prime x \prime x^{\prime}}$ in these new coordinates.

It will turn out in Section 5 that both the Miura transformation as well as the reciprocal link to the Dym equation originates from the construction of these equations as representatives of the three classes (2.28). In fact, the existence of these links is granted by this construction and is in no way related to the fact that these equations are given by the particular second order Lax operators (2.29). As will be shown in Section 5, such links can be established for a whole family of scattering problems representing the three classes in (2.28).

\section{§3. Three Classes of Integrable Equations in $1+1$ Dimensions}

\section{§3.1. Reductions}

As a first step in a more general discussion of the integrable equations (2.28) we have to explain what type of Lax operators may be used in (2.28) to obtain a consistent operator evolution equivalent to some nonlinear integrable equation. In particular, we have to explain why the particular choices of Lax operators (2.29) work. We start by looking a Lax operators $L$ in general position

$$
L=u_{N} \partial^{N}+u_{N-1} \partial^{N-1}+. .+u_{1} \partial+u_{0}+u_{-1} \partial^{-1}+\ldots
$$

of $N$-th order, parametrized by infinitely many fields $u_{N}, u_{N-1}, \ldots$. To obtain a consistent Lax equation we have to ensure that the commutator in (2.28) yields an integro-differential operator of order not exceeding the order $N$ 
of (3.1). Observing $\left[P_{\geq k}\left(L^{q}\right), L\right]=-\left[P_{<k}\left(L^{q}\right), L\right]$ with some $P_{<k}\left(L^{q}\right)=$ $a_{k-1} \partial^{k-1}+a_{k-2} \partial^{k-2}+\cdots$ one immediately obtains the highest order of the commutator as

$$
\begin{aligned}
\frac{d}{d t_{q}} L & =-\left[P_{<k}\left(L^{q}\right), L\right]=-\left[a_{k-1} \partial^{k-1}+\text { lower, } u_{N} \partial^{N}+\text { lower }\right] \\
& =\left(N u_{N}\left(a_{k-1}\right)_{x}-(k-1) a_{k-1}\left(u_{N}\right)_{x}\right) \partial^{N+k-2}+\text { lower }
\end{aligned}
$$

where lower represents lower differential orders. Hence, for the cases $k=0,1,2$ under consideration here, the form of the commutator (3.2) matches the form (3.1) of the Lax operator, and the corresponding time evolution for the fields $u_{N}, u_{N-1}, \ldots$ is obtained from the coefficients of each power of $\partial$ in (3.2). However, we note that for $k=0$ the two highest orders $N$ and $N-1$ are not present in the commutator. As a result the fields $u_{N}$ and $u_{N-1}$ of (3.1) will not inherit any dynamics from the Lax equation (3.2). With other words, the two highest fields will be time-independent functions (of the space variable $x$ ) which can be chosen arbitrarily. For $k=1$ only the highest field $u_{N}$ will inherit a trivial dynamics, for $k=2$ all fields in (3.1) will be dynamical fields. Hence, as a first step, we have obtained some information on the highest orders of the operators admissible for the Lax equations (2.28). They are given in the form

$$
\begin{array}{ll}
\underline{k=0:} & L=c_{N} \partial^{N}+c_{N-1} \partial^{N-1}+u_{N-2} \partial^{N-2}+. .+u_{0}+u_{-1} \partial^{-1}+\cdots, \\
\underline{k=1:} & L=c_{N} \partial^{N}+u_{N-1} \partial^{N-1}+u_{N-2} \partial^{N-2}+. .+u_{0}+u_{-1} \partial^{-1}+\cdots, \\
\underline{k=2:} & L=u_{N} \partial^{N}+u_{N-1} \partial^{N-1}+u_{N-2} \partial^{N-2}+. .+u_{0}+u_{-1} \partial^{-1}+\cdots,
\end{array}
$$

where the $u_{i}$ are dynamical fields and $c_{N}, c_{N-1}$ are arbitrary time-independent functions of $x$. The three hierarchies of equations (2.28) in general position (3.3) are to be interpreted as nonlinear hierarchies of coupled systems for the parametrizing fields $u_{i}$. In this sense (2.28) represents three hierarchies of $1+1$-dimensional equations involving the time variable $t_{q}$ and the space variable $x$ for an infinite number of fields $u_{i}$. For $k=0$ this point of view represents Sato's construction of the KP hierarchy, from which the KP equation itself can be isolated as a $2+1$-dimensional equation. In a similar fashion the modified KP as well as a $2+1$-dimensional Dym equation can be extracted for the cases $k=1$ and $k=2$, respectively. This point of view is adopted in Ref. [24] and will also be discussed in Section 4. At this 
stage we are interested in a different way of extracting closed systems of nonlinear integrable equations for a finite number of fields. Having discussed the properties of the highest orders for the admissible Lax operators, we now discuss reduction possibilities concerning possible lowest differential orders of the operators.

It is readily verified that Lax operators (3.1) in general position can be restricted to the form

$$
\begin{array}{ll}
\underline{k=0:} & L=c_{N} \partial^{N}+c_{N-1} \partial^{N-1}+u_{N-2} \partial^{N-2}+. .+u_{0} \\
\underline{k=1:} & L=c_{N} \partial^{N}+u_{N-1} \partial^{N-1}+u_{N-2} \partial^{N-2}+. .+u_{0}+\partial^{-1} u_{-1}, \\
\underline{k=2:} & L=u_{N} \partial^{N}+u_{N-1} \partial^{N-1}+u_{N-2} \partial^{N-2}+. .+u_{0}+\partial^{-1} u_{-1}+\partial^{-2} u_{-2} .
\end{array}
$$

We point out that here the integration symbols $\partial^{-1}$ and $\partial^{-2}$ turn up on the left of the fields $u_{-1}$ and $u_{-2}$, hence - rewriting these symbols using (2.19) one encounters an "infinite tail" of integration symbols in these operators. It is quite easy to see from a direct computation that operators of the above form do indeed lead to consistent Lax equations of the form (2.28). For instance, for the case $k=0$ the commutator $\left[P_{\geq 0}\left(L^{q}\right), L\right]$ will be a purely differential operator, if $L$ is a purely differential operator. For the case $k=1$ the commutator $\left[P_{\geq 1}\left(L^{q}\right), L\right]$ will be of the form $D_{1}-\partial^{-1} u_{-1} D_{2}$ with differential operators $D_{1,2}$. Here the term $\partial^{-1} u_{-1} D_{2}$ can be rewritten as $\partial^{-1}$ function + differential operator, matching the form of the operator in (3.4). A similar analysis applies to the case $k=2$ as well. As we will see in Section 3.4, the reduction to operators of the form (3.4) can be best understood from the underlying Hamiltonian concept. The operators (3.4) lie in the dual of the subspace $g_{<k}$ given by (2.27). It will be argued in Section 3.4 that the linear Poisson bracket associated with a Lie algebra decomposition can always be properly restricted to the dual subspaces. Hence, all Hamiltonian equations -in particular the equations (2.28) associated with the Casimir functions - can be restricted to the dual subalgebras. As a result, all the nonlinear equations (2.28) with Lax operators of the form (3.4) will inherit a Hamiltonian formulation from the abstract linear bracket.

We remark that for $k=1$ further admissible reductions for the equations (2.28) are given by

$$
\begin{aligned}
& L=c_{N} \partial^{N}+u_{N-1} \partial^{N-1}+u_{N-2} \partial^{N-2}+. .+u_{1} \partial+u_{0}+\partial^{-1} u_{-1}, \\
& L=c_{N} \partial^{N}+u_{N-1} \partial^{N-1}+u_{N-2} \partial^{N-2}+. .+u_{1} \partial+u_{0},
\end{aligned}
$$




$$
L=c_{N} \partial^{N}+u_{N-1} \partial^{N-1}+u_{N-2} \partial^{N-2}+. .+u_{1} \partial+\lambda,
$$

where $\lambda$ is an arbitrary constant parameter. Again, this is easy to see from a direct computation. In the reduction to $u_{-1}=0$ the operator $L$ becomes purely differential and the commutator with $P_{\geq 1}\left(L^{q}\right)$ will be again purely differential. In the last reduction the parameter $\lambda$ has no effect in the commutator $\left[P_{\geq 1}\left(L^{q}\right), L\right]=\left[a_{1} \partial+\right.$ higher, $\lambda+u_{1} \partial+$ higher $]=(..) \partial+$ higher, and the resulting operator matches the form of the Lax operator. As a result, one can immediately predict the following property of the nonlinear equations for $u_{-1}, . ., u_{N-1}$ given by the choice $L=c_{N} \partial^{N}+. .+\partial^{-1} u_{-1}$ : These equations are such that they will admit the reductions $u_{-1}=0$ and $\left(u_{-1}=0\right.$, $u_{0}=$ const $=\lambda$ ). We will see, however, that only the first choice in (3.5) stems from the Hamiltonian reduction mentioned above. The additional reductions to $u_{-1}=0$ and $\left(u_{-1}=0, u_{0}=\lambda\right)$ will leave the particular Hamiltonian equations (2.28) associated with the Casimir functions $C_{q}=\operatorname{tr}\left(L^{q}\right) / q$ invariant, but do not necessarily admit a proper restriction of the Hamiltonian structure.

In a similar way, following reductions

$$
\begin{aligned}
& L=u_{N} \partial^{N}+u_{N-1} \partial^{N-1}+. .+u_{2} \partial^{2}+u_{1} \partial+u_{0}+\partial^{-1} u_{-1}+\partial^{-2} u_{-2}, \\
& L=u_{N} \partial^{N}+u_{N-1} \partial^{N-1}+. .+u_{2} \partial^{2}+u_{1} \partial+u_{0}+\partial^{-1} u_{-1} \\
& L=u_{N} \partial^{N}+u_{N-1} \partial^{N-1}+. .+u_{2} \partial^{2}+u_{1} \partial+u_{0} \\
& L=u_{N} \partial^{N}+u_{N-1} \partial^{N-1}+. .+u_{2} \partial^{2}+u_{1} \partial+\lambda_{1}+\lambda_{2} x \\
& L=u_{N} \partial^{N}+u_{N-1} \partial^{N-1}+. .+u_{2} \partial^{2}+\left(\lambda_{1}+\lambda_{2} x\right) \partial+\lambda_{3}
\end{aligned}
$$

are admissible for the case $k=2$, where all $\lambda$ 's are arbitrary constant parameters. In all these cases it is readily verified that the lowest order of the commutator $\left[P_{\geq 2}\left(L^{q}\right), L\right]$ matches the lowest order of $\frac{d}{d t_{q}} L$ for arbitrary operators $P_{\geq 2}\left(L^{q}\right)=a_{M} \partial^{M}+. .+a_{2} \partial^{2}$. Again, only the first case in (3.6) is a Hamiltonian reduction for the general linear Poisson bracket. All other reductions leave the equations (2.28), $k=2$, invariant, but will not necessarily admit a reduction of the Poisson structure.

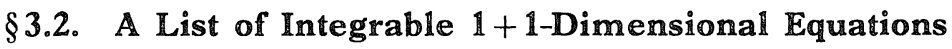

In this section we will display a list of the simplest nonlinear integrable equations encoded in (2.28) by specializing the Lax operator as in (3.4) and 
(3.5)/(3.6), respectively. All equations exhibited in this section are obtained in exactly the same way as the three "prototype" equations $\mathrm{KdV}$, modified $\mathrm{KdV}$ and Dym in Section 2. We consider a $N$ th order operator $L=u_{N} \partial^{N}+$ lower orders. Here the highest coefficient will be chosen as $u_{N}=c_{N}=1$ for the cases $k=0,1$ and $u_{N}=u^{N}$ (with some dynamical field $u$ ) for the case $k=2$, respectively. Then, following the procedure of algebraically calculating the " $N$ th root" as explained in Section 2, one constructs $L^{\frac{1}{N}}=A \partial+a_{0}+a_{1} \partial^{-1}+.$. , where $A=1$ in the cases $k=0,1$ and $A=u$ in the case $k=2$, respectively. Calculating the powers $L^{\frac{n}{N}}$ and applying the projections $P_{\geq k}$ one considers the Lax equations

$$
\frac{d}{d t_{n}} L=\left[P_{\geq k}\left(L^{\frac{n}{N}}\right), L\right], \quad n=1,2,3, . .
$$

For $k=0,1$ the choice $n=1$ will always lead to the dynamics $u_{i t_{1}}=u_{i x}$ for the fields $u_{i}$ in $L$, so that we may identify $t_{1}=x$ in these cases. For $k=0$ and purely differential $L$ the equations become trivial for integer values of $n / N$, because then $P_{\geq 0}\left(L^{\frac{n}{N}}\right)=L^{\frac{n}{N}}$. In the calculations leading to the nonlinear equations the only difficulty arising is of a technical nature. In order to obtain $P_{\geq k}\left(L^{\frac{n}{N}}\right)$ one has to calculate sufficiently many coefficients of the expansion $L^{\frac{1}{N}}=A \partial+\Sigma_{i \leq 0} a_{i} \partial^{i}$ in terms of the fields $u_{i}$ parametrizing the $\operatorname{Lax}$ operators. In particular, the coefficients up to $a_{n-1-k}$ will contribute to the $n$th equation in the hierarchy (3.7). Hence, for large $n$ and $N$, these calculation will become very cumbersome. We have implemented the necessary routines on a computer using the Symbolic Manipulation package Maple ([32]). In the following list of equations we will consider Lax operators up to fifth order. For each choice of $k=0,1$ or 2 and $N$ we will exhibit the first nontrivial of the nonlinear isospectral equations (3.7) associated with the chosen scattering operator.

The case $k=0$ :

As already discussed in Section 2, considering second order Lax operators one obtains

$$
L=\partial^{2}+u, \quad P_{\geq 0}\left(L^{\frac{3}{2}}\right)=\partial^{3}+\frac{3}{2} u \partial+\frac{3}{4} u_{x}
$$


so that (3.7) is equivalent to the $\mathrm{KdV}$ equation $4 u_{t_{3}}=u_{x x x}+6 u u_{x}$ in accordance with the results of Section 2 .

For third order Lax operators one finds

$$
L=\partial^{3}+u \partial+v, \quad P_{\geq 0}\left(L^{\frac{2}{3}}\right)=\partial^{2}+\frac{2}{3} u,
$$

where the fractional power was calculated according to the procedure given in Section 2. The resulting equation is

$$
3\left(\begin{array}{l}
u \\
v
\end{array}\right)_{t_{2}}=\left(\begin{array}{l}
-3 u_{x x}+6 v_{x} \\
-2 u_{x x x}+3 v_{x x}-2 u u_{x}
\end{array}\right) .
$$

As a consequence, the field $u$ satisfies the Boussinesq equation $3 u_{t_{2} t_{2}}+\left(u_{x x x}+\right.$ $\left.4 u u_{x}\right)_{x}=0$.

For 4 th order Lax operators one finds

$$
L=\partial^{4}+u \partial^{2}+v \partial+w, \quad P_{\geq 0}\left(L^{\frac{2}{4}}\right)=\partial^{2}+\frac{1}{2} u
$$

The resulting equation is

$$
2\left(\begin{array}{c}
u \\
v \\
w
\end{array}\right)_{t_{2}}=\left(\begin{array}{l}
-4 u_{x x}+4 v_{x} \\
-4 u_{x x x}+2 v_{x x}+4 w_{x}-2 u u_{x} \\
-4 u_{x x x x}+2 w_{x x}-u u_{x x}-u_{x} v
\end{array}\right)
$$

For 5 th order Lax operators one finds

$$
L=\partial^{5}+u \partial^{3}+v \partial^{2}+w \partial+z, \quad P_{\geq 0}\left(L^{\frac{2}{5}}\right)=\partial^{2}+\frac{2}{5} u
$$

The resulting equation is

$$
5\left(\begin{array}{c}
u \\
v \\
w \\
z
\end{array}\right)_{t_{2}}=\left(\begin{array}{l}
-15 u_{x x}+10 v_{x} \\
-20 u_{x x x}+5 v_{x x}+10 w_{x}-6 u u_{x} \\
-10 u_{x x x x}+5 w_{x x}+10 z_{x}-6 u u_{x x}-4 v u_{x} \\
-2 u_{x x x x x}+5 z_{x x}-2 u u_{x x x}-2 v u_{x x}-2 w u_{x}
\end{array}\right)
$$


The case $k=1$.

For first order Lax operators one obtains

$$
L=\partial+u+\partial^{-1} v, \quad P_{\geq 1}\left(L^{2}\right)=\partial^{2}+2 u \partial
$$

so that (3.7) is equivalent to the system of equations

$$
\left(\begin{array}{l}
u \\
v
\end{array}\right)_{t_{2}}=\left(\begin{array}{c}
u_{x x}+2 v_{x}+2 u u_{x} \\
-v_{x x}+2(u v)_{x}
\end{array}\right)
$$

of Kaup and Broer discussed in Ref. [21]. According to the additional reductions given by (3.5) we can restrict the operators and equations to $v=0$, leading to Burgers' equation

$$
L=\partial+u, \quad P_{\geq 1}\left(L^{2}\right)=\partial^{2}+2 u \partial, \quad u_{t_{2}}=u_{x x}+2 u u_{x}
$$

For second order Lax operators one finds

$$
L=\partial^{2}+u \partial+v+\partial^{-1} w, \quad P_{\geq 1}(L)=\partial^{2}+u \partial
$$

leading to the equation

$$
\left(\begin{array}{c}
u \\
v \\
w
\end{array}\right)_{t_{2}}=\left(\begin{array}{c}
2 v_{x} \\
v_{x x}+2 w_{x}+u v_{x} \\
-w_{x x}+(u w)_{x}
\end{array}\right)
$$

The reduction $w=0$ leads to

$$
\left(\begin{array}{l}
u \\
v
\end{array}\right)_{t_{2}}=\left(\begin{array}{c}
2 v_{x} \\
v_{x x}+u v_{x}
\end{array}\right),
$$

which turns out to be Burgers equation with a time independent driving term, when introducing the variable $f=2 v-u_{x}-\frac{1}{2} u^{2}$ : 


$$
\left(\begin{array}{l}
u \\
f
\end{array}\right)_{t_{2}}=\left(\begin{array}{c}
u_{x x}+u u_{x}+f_{x} \\
0
\end{array}\right)
$$

The next reduction to $v=\lambda$ leaves (3.20) trivial. However, for the next equation in the hierarchy one calculates

$$
L=\partial^{2}+u \partial+v+\partial^{-1} w, \quad P_{\geq 1}\left(L^{\frac{3}{2}}\right)=\partial^{3}+\frac{3}{2} u \partial^{2}+\frac{3}{8}\left(2 u_{x}+u^{2}+4 v\right) \partial .
$$

The resulting equation is

$8\left(\begin{array}{l}u \\ v \\ w\end{array}\right)_{t_{3}}=\left(\begin{array}{l}2 u_{x x x}+12 v_{x x}+24 w_{x}-3 u^{2} u_{x}+12(u v)_{x} \\ 8 v_{x x x}+12 u v_{x x}+6 u_{x} v_{x}+12 u_{x} w+24 u w_{x}+12 v v_{x}+3 u^{2} v_{x} \\ 8 w_{x x x}-12 u w_{x x}-18 u_{x} w_{x}-6 u_{x x} w+12(v w)_{x}+3\left(u^{2} w\right)_{x}\end{array}\right)$,

for which the reduction $w=0, v=\lambda$ leads to the modified $\mathrm{KdV}$ equation

$$
\begin{gathered}
L=\partial^{2}+u \partial+\lambda, \quad P_{\geq 1}\left(L^{\frac{3}{2}}\right)=\partial^{3}+\frac{3}{2} u \partial^{2}+\frac{3}{8}\left(4 \lambda+2 u_{x}+u^{2}\right) \partial, \\
4 u_{t_{3}}=u_{x x x}-\frac{3}{2} u^{2} u_{x}+6 \lambda u_{x}
\end{gathered}
$$

in accordance with the results of Section 2 .

For third order Lax operators one finds

$$
L=\partial^{3}+u \partial^{2}+v \partial+w+\partial^{-1} z, \quad P_{\geq 1}\left(L^{\frac{2}{3}}\right)=\partial^{2}+\frac{2}{3} u \partial
$$

The resulting equation is

$$
3\left(\begin{array}{c}
u \\
v \\
w \\
z
\end{array}\right)_{t_{2}}=\left(\begin{array}{l}
-3 u_{x x}+6 v_{x}-2 u u_{x} \\
-2 u_{x x x}+3 v_{x x}+6 w_{x}+2 u v_{x}-2 u_{x} v-2 u u_{x x} \\
3 w_{x x}+6 z_{x}+2 u w_{x} \\
-3 z_{x x}+2(u z)_{x}
\end{array}\right) .
$$


For the reduction $z=0, w=$ const we obtain a "modified Boussinesq" equation

$$
3\left(\begin{array}{l}
u \\
v
\end{array}\right)_{t_{2}}=\left(\begin{array}{l}
-3 u_{x x}+6 v_{x}-2 u u_{x} \\
-2 u_{x x x}+3 v_{x x}+2 u v_{x}-2 u_{x} v-2 u u_{x x}
\end{array}\right) .
$$

It will be explained in Section 5, how a Miura transformation to the Boussinesq equation (3.10) can be constructed.

For 4th order Lax operators one finds

$$
L=\partial^{4}+u \partial^{3}+v \partial^{2}+w \partial+z+\partial^{-1} r, \quad P_{\geq 1}\left(L^{\frac{2}{4}}\right)=\partial^{2}+\frac{1}{2} u \partial .
$$

The resulting equation is

$$
2\left(\begin{array}{c}
u \\
v \\
w \\
z \\
r
\end{array}\right)_{t_{2}}=\left(\begin{array}{l}
-4 u_{x x}+4 v_{x}-2 u u_{x} \\
-4 u_{x x x}+2 v_{x x}+4 w_{x}-2 u_{x} v+u v_{x}-3 u u_{x x} \\
-4 u_{x x x x}+2 w_{x x}+4 z_{x}-u_{x} w+u w_{x}-u u_{x x x}-v u_{x x} \\
2 z_{x x}+4 v_{x}+u z_{x} \\
-2 r_{x x}+(u r)_{x}
\end{array}\right)
$$

For the reduction $r=0, z=$ const we obtain a "modified" version of equation (3.12)

$$
2\left(\begin{array}{l}
u \\
v \\
w
\end{array}\right)_{t_{2}}=\left(\begin{array}{l}
-4 u_{x x}+4 v_{x}-2 u u_{x} \\
-4 u_{x x x}+2 v_{x x}+4 w_{x}-2 u_{x} v+u v_{x}-3 u u_{x x} \\
-4 u_{x x x x}+2 w_{x x}-u_{x} w+u w_{x}-u u_{x x x}-v u_{x x}
\end{array}\right)
$$

For 5 th order Lax operators one finds

$$
L=\partial^{5}+u \partial^{4}+v \partial^{3}+w \partial^{2}+z \partial+r+\partial^{-1} s, \quad P_{\geq 1}\left(L^{\frac{2}{5}}\right)=\partial^{2}+\frac{2}{5} u \partial .
$$

The resulting equation is 


$$
5\left(\begin{array}{c}
u \\
v \\
w \\
z \\
r \\
s
\end{array}\right)_{t_{2}}\left(\begin{array}{l}
-15 u_{x x}+10 v_{x}-6 u u_{x} \\
-20 u_{x x x}+5 v_{x x}+10 w_{x}-12 u u_{x x}-6 u_{x} v+2 u v_{x} \\
-10 u_{x x x x}+5 w_{x x}+10 z_{x}-8 u u_{x x x}-6 u_{x x} v-4 u_{x} w+2 u w_{x} \\
-2 u_{x x x x x}+5 z_{x x}+10 r_{x}-2 u u_{x x x x}-2 u_{x x x} v-2 u_{x x} w- \\
5 r_{x x}+10 s_{x}+2 u r_{x} \\
-5 s_{x x}+2(u s)_{x}
\end{array}\right)
$$

For the reduction $s=0, r=$ const we obtain a "modified" version of equation (3.14)

$$
5\left(\begin{array}{l}
u \\
v \\
w \\
z
\end{array}\right)_{t_{2}}=\left(\begin{array}{l}
-15 u_{x x}+10 v_{x}-6 u u_{x} \\
-20 u_{x x x}+5 v_{x x}+10 w_{x}-12 u u_{x x}-6 u_{x} v+2 u v_{x} \\
-10 u_{x x x x}+5 w_{x x}+10 z_{x}-8 u u_{x x x}-6 u_{x x} v-4 u_{x} w+2 u w_{x} \\
-2 u_{x x x x x}+5 z_{x x}-2 u u_{x x x x}-2 u_{x x x} v-2 u_{x x} w-2 u_{x} z+2 u z_{x}
\end{array}\right) .
$$

The case $k=2$.

For first order Lax operators one obtains

$$
L=u \partial+v+\partial^{-1} w+\partial^{-2} z, \quad P_{\geq 2}\left(L^{2}\right)=u^{2} \partial^{2},
$$

so that (3.7) is equivalent to the system of equations

$$
\left(\begin{array}{c}
u \\
v \\
w \\
z
\end{array}\right)_{t_{2}}=\left(\begin{array}{c}
u^{2} u_{x x}+2 u^{2} v_{x} \\
u^{2} v_{x x}+2 u(u w)_{x} \\
-\left(u^{2} w\right)_{x x}+2\left(u^{2} z\right)_{x} \\
-\left(u^{2} z\right)_{x x}
\end{array}\right)
$$

According to the additional reductions given by (3.6) we can restrict the operators and equations to 


$$
\begin{gathered}
\left(\begin{array}{c}
u \\
v \\
w
\end{array}\right)_{t_{2}}=\left(\begin{array}{c}
u^{2} u_{x x}+2 u^{2} v_{x} \\
u^{2} v_{x x}+2 u(u w)_{x} \\
-\left(u^{2} w\right)_{x x}
\end{array}\right),\left(\begin{array}{l}
u \\
v
\end{array}\right)_{t_{2}}=\left(\begin{array}{l}
u^{2} u_{x x}+2 u^{2} v_{x} \\
u^{2} v_{x x}
\end{array}\right), \\
u_{t_{2}}=u^{2} u_{x x}+2 \lambda u^{2} .
\end{gathered}
$$

For the last reduction we have used $z=w=0, v=\lambda x$.

For second order Lax operators one finds

$$
L=u^{2} \partial^{2}+v \partial+w+\partial^{-1} z+\partial^{-2} r, \quad \mathbb{P}_{\geq 2}(L)=u^{2} \partial^{2}
$$

leading to the equation

$$
\left(\begin{array}{c}
u \\
v \\
w \\
z \\
v
\end{array}\right)_{t_{2}}=\left(\begin{array}{c}
u v_{x}-v u_{x} \\
u^{2} v_{x x}+2 u^{2} w_{x} \\
u^{2} w_{x x}+2 u(u z)_{x} \\
-\left(u^{2} z\right)_{x x}+2\left(u^{2} v\right)_{x} \\
-\left(u^{2} r\right)_{x x}
\end{array}\right)
$$

The reductions according to (3.6) lead to

$$
\left(\begin{array}{c}
u \\
v \\
w \\
z
\end{array}\right)_{t_{2}}=\left(\begin{array}{c}
u v_{x}-v u_{x} \\
u^{2} v_{x x}+2 u^{2} w_{x} \\
u^{2} w_{x x}+2 u(u z)_{x} \\
-\left(u^{2} z\right)_{x x}
\end{array}\right),\left(\begin{array}{c}
u \\
v \\
w
\end{array}\right)_{t_{2}}=\left(\begin{array}{l}
u v_{x}-v u_{x} \\
u^{2} v_{x x}+2 u^{2} w_{x} \\
u^{2} w_{x x}
\end{array}\right)
$$

for $r=0$ and $r=z=0$. Choosing $w=\lambda x$ and $(v=\lambda x, w=$ const), respectively, one can reduce (3.39) to the following equations

$$
\left(\begin{array}{l}
u \\
v
\end{array}\right)_{t_{2}}=\left(\begin{array}{l}
u v_{x}-v u_{x} \\
u^{2} v_{x x}+2 \lambda u^{2}
\end{array}\right), \quad u_{t_{2}}=\lambda\left(u-x u_{x}\right) .
$$

For the very last reduction the evolution equation for $u$ has become linear. It is the first member of the Dym hierarchy, its solution describes a scaling of the variables. Indeed, for the next equation in the hierarchy one calculates 


$$
L=u^{2} \partial^{2}+v \partial+w+\partial^{-1} z+\partial^{-2} r, \quad P_{\geq 2}\left(L^{\frac{3}{2}}\right)=u^{3} \partial^{3}+\frac{3}{2}\left(u^{2} u_{x}+u v\right) \partial^{2} .
$$

The resulting equation for general $(u, v, w, z, r)$ is rather complicated, we only write down its reduction to $z=r=0$ :

$$
8\left(\begin{array}{l}
u \\
v \\
w
\end{array}\right)_{t_{3}}=\left(\begin{array}{l}
2 u^{3} u_{x x x}+6 u^{2} v_{x x}+12 u^{2} w_{x}+3 u^{2}\left(u^{-2} v^{2}\right)_{x} \\
8 u^{3} v_{x x x}+4\left(u^{3}\right)_{x} v_{x x}+24 u^{2}\left(u w_{x}\right)_{x}+12 u v v_{x x}+24 u v w_{x} \\
8 u^{3} w_{x x x}+4\left(u^{3}\right)_{x} w_{x x}+12 u v w_{x x}
\end{array}\right)
$$

The reduction $w=\lambda x$ leads to the integrable equation

$$
8\left(\begin{array}{l}
u \\
v
\end{array}\right)_{t_{3}}=\left(\begin{array}{l}
2 u^{3} u_{x x x}+6 u^{2} v_{x x}+3 u^{2}\left(u^{-2} v^{2}\right)_{x}+12 \lambda u^{2} \\
8 u^{3} v_{x x x}+4\left(u^{3}\right)_{x} v_{x x}+12 u v v_{x x}+24 \lambda\left(u^{2} u_{x}+u v\right)
\end{array}\right)
$$

which is the next equation in the hierarchy associated with the equation (3.40.i), related to the scattering operator $L=u^{2} \partial^{2}+v \partial+\lambda x$. Imposing the reduction $w=$ const, $v=\lambda x$ on (3.42) one obtains the Dym equation

$$
4 u_{t_{3}}=u^{3} u_{x x x}+3 \lambda^{2} x \frac{u-x u_{x}}{u}
$$

in accordance with the results of Section 2 .

For third order Lax operators one finds

$$
L=u^{3} \partial^{3}+v \partial^{2}+w \partial+z+\partial^{-1} r+\partial^{-2} s, \quad P_{\geq 2}\left(L^{\frac{2}{3}}\right)=u^{2} \partial^{2} .
$$

We only exhibit the resulting equation for the choice $z=r=s=0, w=\lambda x$ :

$$
3\left(\begin{array}{l}
u \\
v
\end{array}\right)_{t_{2}}=\left(\begin{array}{c}
-3 u^{2} u_{x x}+2 u^{2}\left(u^{-2} v\right)_{x} \\
3 u^{2} v_{x x}-3 v\left(u^{2}\right)_{x x}-6 u\left(u^{3} u_{x x}\right)_{x}+6 \lambda u\left(u-x u_{x}\right)
\end{array}\right)
$$

We found that the next equation in this hierarchy admits an additional reduction to $v=w=z=r=s=0$, so that one discovers the simple scalar fifth order equation 


$$
L=u^{3} \partial^{3}, \quad P_{\geq 2}\left(L^{\frac{5}{3}}\right)=\partial u^{5} \partial^{4}+\frac{5}{3} u^{3}\left(u^{2}\right)_{x x} \partial^{3}, \quad 18 u_{t_{5}}+u^{4}\left(u^{2}\right)_{x x x x x}=0
$$

which is related to the equation discussed in Ref. [33]. We observe that no second order term is present in the projected operator $P_{\geq 2}\left(L^{\frac{5}{3}}\right)$. However, there does not seem to be a structural reason for this phenomenon, explaining why the additional constraint to $v=0$ is admissible for the fifth order equation.

For 4 th order Lax operators one finds

$$
L=u^{4} \partial^{4}+v \partial^{3}+w \partial^{2}+z \partial+r+\partial^{-1} s+\partial^{-2} f, \quad P_{\geq 2}\left(L^{\frac{2}{4}}\right)=u^{2} \partial^{2}
$$

We only exhibit the resulting equation for the choice $r=s=f=0, z=\lambda x$ :

$$
2\left(\begin{array}{l}
u \\
v \\
w
\end{array}\right)_{t_{2}}=\left(\begin{array}{l}
-4 u^{2} u_{x x}+u^{2}\left(u^{-3} v\right)_{x} \\
-16 u^{2}\left(u^{3} u_{x x}\right)_{x}+2 u^{2} v_{x x}-6 v\left(u^{2}\right)_{x x}+4 u^{4}\left(u^{-2} w\right)_{x} \\
-2 u^{4}\left(u^{2}\right)_{x x x x}-2 v\left(u^{2}\right)_{x x x}-2 w\left(u^{2}\right)_{x x}+2 u^{2} w_{x x}+4 \lambda u\left(u-x u_{x}\right)
\end{array}\right)
$$

No equations associated with operators of order $N>4$ shall be presented, as even the simplest equations in the hierarchies are of an extremely complicated form.

\section{§3.3. A Discrete Invariance and Kupershmidt Reductions}

For any given Lax formulation $\frac{d}{d t} L=[B, L]$ encoding a nonlinear integrable equation one can use an equivalent Lax formulation in terms of the formally transposed pair $\widetilde{L}=L^{\dagger}$ and $\widetilde{B}=-B^{\dagger}$, which again satisfy $\frac{d}{d t} \tilde{L}=$ $[\tilde{B}, \tilde{L}]$. In terms of the Lax operators (2.18) the transposition is given by

$$
\left(\sum_{i} u_{i} \partial^{i}\right)^{\dagger}=\sum_{i}(-1)^{i} \partial^{i} u_{i}
$$

We now discuss the invariance of the Lax equations $(2.28) /(3.7)$ with respect to transposition of $L$. It is readily seen that 


$$
\left(P_{\geq 0}(A)\right)^{\dagger}=P_{\geq 0}\left(A^{\dagger}\right), \quad\left(P_{<0}(A)\right)^{\dagger}=P_{<0}(A)^{\dagger}
$$

for any operator $A \in g$. Further, observing $P_{\geq k}(A)=P_{\geq 0}\left(A \partial^{-k}\right) \partial^{k}$, one derives the identity

$$
\partial^{-k}\left(P_{\geq k}(A)\right)^{\dagger} \partial^{k}=P_{\geq k}\left(\partial^{-k} A^{\dagger} \partial^{k}\right)
$$

For a given $N$ th order operator $L=A^{N} \partial^{N}+$ lower orders all integrable equations discussed in the last section were constructed via the " $N$ th root"

$$
L^{\frac{1}{N}}=A \partial+a_{0}+a_{1} \partial^{-1}+.
$$

from the condition $\left(L^{\frac{1}{N}}\right)^{N}=L$. All coefficients $a_{0}, a_{1}, \ldots$, in this ansatz are determined uniquely from this condition, once the leading order term $A$ is fixed. The isospectral equations then were obtained using (integer) powers of $L^{\frac{1}{N}}$. We now consider transposed operators

$$
\begin{aligned}
\tilde{L}^{\frac{1}{N}} & =-\partial^{-k}\left(L^{\frac{1}{N}}\right)^{\dagger} \partial^{k}=-\partial^{-k}(A \partial+\text { lower })^{\dagger} \partial^{k}=\partial^{-k}(\partial A+\text { lower }) \partial^{k} \\
& =A \partial+\text { lower },
\end{aligned}
$$

having the same leading order term $A$ as the original $L^{\frac{1}{N}}$. This is exactly the operator representing the " $N$ th root" of the operator

$$
\tilde{L}=(-1)^{N} \partial^{-k} L^{\dagger} \partial^{k}=(-1)^{N} \partial^{-k}\left(a^{N} \partial^{N}+\text { lower }\right)^{\dagger} \partial^{k}=A^{N} \partial^{N}+\text { lower }
$$

in this construction. Using (3.52) the time evolution for $\tilde{L}$ is obtained from (3.7) as

$$
\frac{d}{d t_{n}} \tilde{L}=(-1)^{N} \partial^{-k}\left(\left[P_{\geq k}\left(\left(L^{\frac{1}{N}}\right)^{n}\right), L\right]\right)^{\dagger} \partial^{k}=(-1)^{n+1}\left[P_{\geq k}\left(\left(\tilde{L}^{\frac{1}{N}}\right)^{n}\right), \tilde{L}\right]
$$

Hence, up to the sign $(-1)^{n+1}$, the construction (3.7) of all the equations discussed here is invariant with respect to transposition of the Lax operators. Absorbing the signs into the time variable we have the result that all equations (3.7) are invariant under the discrete transformation

$$
L \quad \rightarrow \quad \widetilde{L}=(-1)^{N} \partial^{-k} L^{\dagger} \partial^{k}
$$




$$
\begin{aligned}
L^{\frac{1}{N}} \rightarrow \tilde{L}^{\frac{1}{N}} & =-\partial^{-k}\left(L^{\frac{1}{N}}\right)^{\dagger} \partial^{k}, \\
t_{n} \rightarrow \tilde{t}_{n} & =\left\{\begin{aligned}
t_{n}, & \text { nodd }, \\
-t_{n}, & \text { neven } .
\end{aligned}\right.
\end{aligned}
$$

Using this result certain discrete invariances for the nonlinear equations can be extracted directly from the Lax operator. For instance, the Boussinesq equation (3.10) is associated with the third order operator $L=\partial^{3}+u \partial+v$, constructed via $P_{\geq 2}\left(L^{\frac{2}{3}}\right)$. Considering

$$
\tilde{L}=\partial^{3}+\tilde{u} \partial+\tilde{v}=-L^{\dagger}=\partial^{3}+\partial u-v=\partial^{3}+u \partial+u_{x}-v
$$

we immediately obtain the invariance

$$
u \rightarrow \tilde{u}=u, \quad v \rightarrow \tilde{v}=-v+u_{x}, \quad t_{2} \rightarrow \tilde{t}_{2}=-t_{2}
$$

for (3.10). The same analysis for the "modified" Boussinesq equation (3.27), associated with the operators $L=\partial^{3}+u \partial^{2}+v \partial, P_{\geq 1}\left(L^{\frac{2}{3}}\right)$, leads to

$$
\tilde{L}=\partial^{3}+\tilde{u} \partial^{2}+\tilde{v} \partial=-\partial^{-1} L^{\dagger} \partial=\partial^{3}-u \partial^{2}+\left(v-u_{x}\right) \partial .
$$

Hence we obtain the invariance

$$
u \rightarrow \tilde{u}=-u, \quad v \rightarrow \tilde{v}=v-u_{x}, \quad t_{2} \rightarrow \tilde{t}_{2}=-t_{2}
$$

for (3.27). As a further example, also the simple invariance $u \rightarrow \tilde{u}=-u$ of the modified $\mathrm{KdV}$ (3.24) is a consequence of the transformation

$$
L=\partial^{2}+u \partial+\lambda \rightarrow \tilde{L}=\partial^{2}+\tilde{u} \partial+\lambda=\partial^{-1} L^{\dagger} \partial=\partial^{2}-u \partial+\lambda
$$

of the Lax operator.

As an important consequence of these symmetry transformations, one may conclude that for odd $n$, that is $\tilde{t}_{n}=t_{n}$, the fixed points $L=\tilde{L}$ of the transformation (3.57) are invariant with respect to the dynamics (3.7). In fact, for any dynamical system $\frac{d}{d t} L=K(L)$ - given by some vectorfield $K$ and a symmetry transformation $L \rightarrow \tilde{L}$, one has $\frac{d}{d t}(L-\tilde{L})=K(L)-K(\tilde{L})=0$ for $L=\tilde{L}$. Hence, the dynamics is tangent to the set of fixed points of the symmetry transformation. For even $n$ the conditions for fixed points of the 
transformation (3.57) include $t_{n}=-t_{n}$, so that the evolution equations associated with even times $t_{n}$ will not admit a reduction to the Lax operators which are invariant with respect to the transformation (3.57). However, no such obstacle arises for the flows associated with the odd times $t_{n}$. As a result, we may restrict each second equation in the three hierarchies (3.7) (with odd $n$ ) to Lax operators satisfying the constraint

$$
L=(-1)^{N} \partial^{-k} L^{\dagger} \partial^{k}
$$

This constraint was discussed by Kupershmidt in Ref. [21] and shall be referred to as Kupershmidt reduction in the following.

We present a list of the first nontrivial equations of the hierarchies associated with operators (3.4) up to fifth order. Again, the arbitrary functions are chosen as $c_{N}(x) \equiv 1$ and $c_{N-1}(x) \equiv 0$ for simplicity.

The case $k=0$.

The second order operator $L=\partial^{2}+u$ associated with the $\mathrm{KdV}$ hierarchy satisfies the Kupershmidt constraint (3.63). Imposing this constraint on third order operators $L=\partial^{3}+u_{1} \partial+u_{0}$ one finds the condition $u_{0}=\frac{1}{2} u_{1 x}$. This reduction cannot be imposed on the Boussinesq equation (3.10), because it represents the flow associated with the even time $t=t_{2}$. The next admissible index $n=3$ leads to a trivial flow for third order operators, the flow associated with $t_{4}$ does not admit the Kupershmidt reduction. Hence, we have to choose $n=5$ to find the first nontrivial flow associated with third order operators constrained by (3.63). Putting $u_{1}=2 u$, one finds

$$
\begin{aligned}
L=\partial^{3}+\partial u+u \partial, P_{\geq 0}\left(L^{\frac{5}{3}}\right)=\partial^{5}+ & \frac{10}{9}\left(\partial^{3} u+u \partial^{3}\right)+\frac{5}{9}\left(\partial^{2} u \partial+\partial u \partial^{2}\right) \\
& +\frac{10}{9}\left(\partial u^{2}+u^{2} \partial\right),
\end{aligned}
$$

leading to the Kaup-Kupershmidt equation ([25])

$$
9 u_{t_{5}}+u_{x x x x x}+10 u u_{x x x}+25 u_{x} u_{x x}+20 u^{2} u_{x}=0 .
$$

It represents a reduction of the $t_{5}$-flow in the Boussinesq hierarchy. 
For 4 th order Lax operators $L=\partial^{4}+u_{2} \partial^{2}+u_{1} \partial+u_{0}$ the constraint (3.63) leads to the condition $u_{1}=u_{2 x}$. With $u=u_{2}, v=u_{0}$, one finds

$$
L=\partial^{4}+\partial u \partial+v, \quad P_{\geq 0}\left(L^{\frac{3}{4}}\right)=\partial^{3}+\frac{3}{8}(\partial u+u \partial),
$$

leading to

$$
8\left(\begin{array}{l}
u \\
v
\end{array}\right)_{t_{3}}=\left(\begin{array}{l}
-10 u_{x x x}-6 u u_{x}+24 v_{x} \\
-3\left(u_{x x x x x}+u u_{x x x}+u_{x} u_{x x}\right)+8 v_{x x x}+6 u v_{x}
\end{array}\right) .
$$

For 5 th order Lax operators $L=\partial^{5}+u_{3} \partial^{3}+u_{2} \partial^{2}+u_{1} \partial+u_{0}$ the constraint (3.63) leads to the conditions $u_{2}=\frac{3}{2} u_{3 x}, \quad u_{0}=-\frac{1}{4} u_{3 x x x}+\frac{1}{2} u_{1 x}$. With $u=\frac{1}{2} u_{3}$, $v=-\frac{3}{4} u_{3 x x}+\frac{1}{2} u_{1}$, one finds

$$
L=\partial^{5}+\partial^{3} u+u \partial^{3}+\partial v+v \partial, \quad P_{\geq 0}\left(L^{\frac{3}{5}}\right)=\partial^{3}+\frac{3}{5}(\partial u+u \partial)
$$

leading to

$$
5\left(\begin{array}{l}
u \\
v
\end{array}\right)_{t_{3}}=\left(\begin{array}{c}
5 u_{x x x}+15 v_{x}-12 u u_{x} \\
-3 u_{x x x x x}-10 v_{x x x}+12 u u_{x x x}+27 u_{x} u_{x x}+6 u v_{x}-6 u_{x} v
\end{array}\right)
$$

The case $k=1$.

Imposing (3.63) on first order operators $L=\partial+u_{0}+\partial^{-1} u_{-1}$ one finds the constraint $u_{0}=0$. With $u=u_{-1}$ one calculates

$$
L=\partial+\partial^{-1} u, \quad P_{\geq 1}\left(L^{3}\right)=\partial^{3}+3 u \partial
$$

leading to the $\mathrm{KdV}$ equation

$$
u_{t_{3}}=u_{x x x}+6 u u_{x}
$$

The fact that the $\mathrm{KdV}$ hierarchy can be represented in the class $k=1$ using the operator $L=\partial+\partial^{-1} u$ was discussed by Kupershmidt ([21]) (using the transposed operators). 
For second order operators $L=\partial^{2}+u_{1} \partial+u_{0}+\partial^{-1} u_{-1}$ the Kupershmidt constraint leads to $u_{1}=0, u_{-1}=-\frac{1}{2} u_{0 x}$. Putting $u_{0}=2 u$ one finds

$$
L=\partial^{2}+u+\partial^{-1} u \partial, \quad P_{\geq 1}\left(L^{\frac{3}{2}}\right)=\partial^{3}+3 u \partial,
$$

again leading to the $\mathrm{KdV}$ equation

$$
u_{t_{3}}=u_{x x x}+3 u u_{x}
$$

In this case the Lax operator may be regarded as the transposed of the well-known recursion operator of the $\mathrm{KdV}$ hierarchy, when identifying the pseudo-differential symbol $\partial^{-1}$ with the integration operator $D^{-1}=\int^{x}$.

For third order operators $L=\partial^{3}+u_{2} \partial^{2}+u_{1} \partial+u_{0}+\partial^{-1} u_{-1}$ the Kupershmidt reduction leads to the conditions $u_{2}=u_{0}=0$. With $u=u_{1}, v=u_{-1}$, one finds

$$
L=\partial^{3}+u \partial+\partial^{-1} v, \quad P_{\geq 1}(L)=\partial^{3}+u \partial,
$$

leading to

$$
\left(\begin{array}{l}
u \\
v
\end{array}\right)_{t_{3}}=\left(\begin{array}{c}
3 v_{x} \\
v_{x x x}+(u v)_{x}
\end{array}\right) .
$$

We can intersect the Kupershmidt constraint with the additional reductions given by (3.5). Assuming $v=0$ equation (3.75) becomes trivial. However, passing to the next nontrivial flow admitting the Kupershmidt constraint, one finds

$$
L=\partial^{3}+u \partial, \quad P_{\geq 1}\left(L^{\frac{5}{3}}\right)=\partial^{5}-\frac{5}{9} \partial u \partial^{2}+\frac{10}{9}\left(\partial^{2} u \partial+u \partial^{3}\right)+\frac{5}{9} u^{2} \partial
$$

leading to the Sawada-Kotera equation ([26])

$$
9 u_{t_{5}}+u_{x x x x x}+5 u u_{x x x}+5 u_{x} u_{x x}+5 u^{2} u_{x}=0
$$

It represents the reduction $v=0$ of the $t_{5}$-flow of the hierarchy associated 
with (3.75).

For 4 th order Lax operators $L=\partial^{4}+u_{3} \partial^{3}+u_{2} \partial^{2}+u_{1} \partial+u_{0}+\partial^{-1}$ the constraint (3.63) leads to the conditions $u_{3}=0, u_{1}=\frac{1}{2} u_{2 x}, u_{-1}=\frac{1}{2} u_{0 x}$. With $u_{2}=2 u$, $u_{0}=2 v$ one finds

$$
L=\partial^{4}+\partial u \partial+u \partial^{2}+v+\partial^{-1} v \partial, \quad P_{\geq 1}\left(L^{\frac{3}{4}}\right)=\partial^{3}+\frac{3}{2} u \partial
$$

leading to

$$
2\left(\begin{array}{l}
u \\
v
\end{array}\right)_{t_{3}}=\left(\begin{array}{c}
-u_{x x x}+6 v_{x}-3 u u_{x} \\
2 v_{x x x}+3 u v_{x}
\end{array}\right)
$$

Intersecting with the additional reductions of (3.5), that is, assuming $v=0$, we again find the $\mathrm{KdV}$ equation

$$
L=\partial^{4}+\partial u \partial+u \partial^{2}, \quad P_{\geq 1}\left(L^{\frac{3}{4}}\right)=\partial^{3}+\frac{3}{2} u \partial, \quad 2 u_{t_{3}}+u_{x x x}+3 u u_{x}=0
$$

For 5 th order Lax operators $L=\partial^{5}+u_{4} \partial^{4}+u_{3} \partial^{3}+u_{2} \partial^{2}+u_{1} \partial+u_{0}+\partial^{-1} u_{-1}$ the Kupershmidt constraint leads to the conditions $u_{4}=u_{0}=0, u_{2}=u_{3 x}$. With $u=u_{3}, v=u_{1}, w=u_{-1}$ one finds

$$
L=\partial^{5}+\partial u \partial^{2}+v \partial+\partial^{-1} w, \quad P_{\geq 1}\left(L^{\frac{3}{5}}\right)=\partial^{3}+\frac{3}{5} u \partial
$$

leading to

$$
5\left(\begin{array}{l}
u \\
v \\
w
\end{array}\right)_{t_{3}}=\left(\begin{array}{l}
-10 u_{x x x}+15 v_{x}-6 u u_{x} \\
-3 u_{x x x x x}+5 v_{x x x}+15 w_{x}-3 u u_{x x x}-3 u_{x} u_{x x}+3 u v_{x}-3 u_{x} v \\
-10 w_{x x x}+3(u w)_{x}
\end{array}\right) .
$$

Intersecting with the additional reductions of (3.5), that is, assuming $w=0$, we find the system 


$$
\begin{gathered}
L=\partial^{5}+\partial u \partial^{2}+v \partial, \quad P_{\geq 1}\left(L^{\frac{3}{5}}\right)=\partial^{3}+\frac{3}{5} u \partial \\
5\left(\begin{array}{l}
u \\
v
\end{array}\right)_{t_{3}}=\left(\begin{array}{l}
-10 u_{x x x}+15 v_{x}-6 u u_{x} \\
-3 u_{x x x x x}+5 v_{x x x}-3 u u_{x x x}-3 u_{x} u_{x x}+3 u v_{x}-3 u_{x} v
\end{array}\right) .
\end{gathered}
$$

The case $k=2$.

Imposing (3.63) on first order operators $L=u_{1} \partial+u_{0}+\partial^{-1} u_{-1}+\partial^{-2} u_{-2}$ one finds the constraints $u_{0}=-\frac{1}{2} u_{1}, u_{-2}=\frac{1}{4} u_{1 x x x}-\frac{1}{2} u_{-1 x}$. With $u_{1}=2 u, u_{-1}=$ $u_{x x}+2 v$, one calculates

$$
L=u \partial+\partial^{-1} u \partial^{2}+\partial^{-1} v+\partial^{-2} v \partial, \quad P_{\geq 2}\left(L^{3}\right)=4\left(u^{3} \partial^{3}+\partial u^{3} \partial^{2}\right),
$$

leading to the equation

$$
\left(\begin{array}{l}
u \\
v
\end{array}\right)_{t_{3}}=4\left(\begin{array}{l}
2 u^{3} u_{x x x}+6 u^{2}(u v)_{x}+3 u^{2} u_{x} u_{x x} \\
2 u^{3} v_{x x x}+6 u^{2} u_{x}^{2} v_{x}+3 u^{2} u_{x x} v_{x}+9 u^{2} u_{x} v_{x x}
\end{array}\right)
$$

We can intersect the Kupershmidt constraint with the additional reductions given by (3.6). We may assume $v=\frac{1}{2} \lambda=$ const and find

$$
\begin{gathered}
L=u \partial+\partial^{-1} u \partial^{2}+\lambda \partial^{-1}, \quad P_{\geq 2}\left(L^{3}\right)=4\left(u^{3} \partial^{3}+\partial u^{3} \partial^{2}\right), \\
u_{t_{3}}=4\left(2 u^{3} u_{x x x}+3 u^{2} u_{x} u_{x x}+\lambda\left(u^{3}\right)_{x}\right) .
\end{gathered}
$$

An alternative Lax pair $\tilde{L}_{t_{3}}=[\tilde{B}, \tilde{L}]$ for this last equation is given by the gauge equivalent $\tilde{L}=\partial L \partial^{-1}=\partial u+u \partial+\lambda \partial^{-1}$ and $\widetilde{B}=\partial P_{\geq 2}\left(L^{\frac{3}{2}}\right) \partial^{-1}=4\left(\partial^{2} u^{3} \partial+\right.$ $\left.\partial u^{3} \partial^{2}\right)$

For second order operators $L=u_{2} \partial^{2}+u_{1} \partial+u_{0}+\partial^{-1} u_{-1}+\partial^{-2} u_{-2}$ the Kupershmidt constraint leads to $u_{1}=0, u_{-1}=-u_{0 x}$. Putting $u_{2}=u^{2}, u_{0}=v, u_{-2}=w$ one finds

$$
L=u^{2} \partial^{2}+\partial^{-1} v \partial+\partial^{-2} w, \quad P_{\geq 2}\left(L^{\frac{3}{2}}\right)=\frac{1}{2}\left(u^{3} \partial^{3}+\partial u^{3} \partial^{2}\right),
$$


leading to the equation

$$
4\left(\begin{array}{c}
u \\
v \\
w
\end{array}\right)_{t_{3}}=\left(\begin{array}{l}
u^{3} u_{x x x}+6 u^{3} v_{x} \\
4 u^{3} v_{x x x}+6\left(u^{3}\right)_{x} v_{x x}+2\left(u^{3}\right)_{x x} v_{x}+8\left(u^{3}\right)_{x} w+12 u^{3} w_{x} \\
4 u^{3} w_{x x x}+10\left(u^{3}\right)_{x} w_{x x}+8\left(u^{3}\right)_{x x} w_{x}+2\left(u^{3}\right)_{x x x} w
\end{array}\right)
$$

We can intersect the Kupershmidt constraint with the additional reductions given by (3.6). Assuming $w=0$ we find

$$
L=u^{2} \partial^{2}+\partial^{-1} v \partial, \quad P_{\geq 2}\left(L^{\frac{3}{2}}\right)=\frac{1}{2}\left(u^{3} \partial^{3}+\partial u^{3} \partial^{2}\right)
$$

leading to the equation

$$
4\left(\begin{array}{l}
u \\
v
\end{array}\right)_{t_{3}}=\left(\begin{array}{l}
u^{3} u_{x x x}+6 u^{3} v_{x} \\
4 u^{3} v_{x x x}+6\left(u^{3}\right)_{x} v_{x x}+2\left(u^{3}\right)_{x x} v_{x}
\end{array}\right)
$$

The additional reduction $v=\lambda$ leads to the Dym equation

$$
L=u^{2} \partial^{2}+\lambda, \quad P_{\geq 2}\left(L^{\frac{3}{2}}\right)=\frac{1}{2}\left(u^{3} \partial^{3}+\partial u^{3} \partial^{2}\right), \quad 4 u_{t}=u^{3} u_{x x x}
$$

For third order operators $L=u_{3} \partial^{3}+u_{2} \partial^{2}+u_{1} \partial+u_{0}+\partial^{-1} u_{-1}+\partial^{-2} u_{-2}$ the Kupershmidt reduction leads to the conditions $u_{2}=\frac{1}{2} u_{3 x}, u_{0}=-\frac{1}{2} u_{1 x}$, $u_{-2}=\frac{1}{4} u_{1 x x x}-\frac{1}{2} u_{-1 x}$. With $u_{3}=2 u^{3}, u_{1}=2 v, u_{-1}=v_{x x}+2 w$, one finds

$$
L=u^{3} \partial^{3}+\partial u^{3} \partial^{2}+v \partial+\partial^{-1} v \partial^{2}+\partial^{-1} w+\partial^{-2} w \partial, \quad P_{\geq 2}(L)=u^{3} \partial^{3}+\partial u^{3} \partial^{2}
$$

leading to

$$
\left(\begin{array}{l}
u \\
v \\
w
\end{array}\right)_{t_{3}}=\left(\begin{array}{l}
2 u v_{x}-2 u_{x} v \\
2 u^{3} v_{x x x}+\left(u^{3}\right)_{x} v_{x x}+6 u^{3} w_{x}+2\left(u^{3}\right)_{x} w \\
2 u^{3} w_{x x x}+3\left(u^{3}\right)_{x} w_{x x}+\left(u^{3}\right)_{x x} w_{x}
\end{array}\right)
$$


We can intersect the Kupershmidt constraint with the additional reductions given by (3.6). Assuming $w=\lambda=$ const equation (3.93) becomes

$$
\begin{gathered}
L=u^{3} \partial^{3}+\partial u^{3} \partial^{2}+v \partial+\partial^{-1} v \partial^{2}, \quad P_{\geq 2}(L)=u^{3} \partial^{3}+\partial u^{3} \partial^{2} \\
\left(\begin{array}{l}
u \\
v
\end{array}\right)_{t_{3}}=\left(\begin{array}{l}
2 u v_{x}-2 u_{x} v \\
2 u^{3} v_{x x x}+\left(u^{3}\right)_{x} v_{x x}+2 \lambda\left(u^{3}\right)_{x}
\end{array}\right)
\end{gathered}
$$

An alternative (purely differential) Lax pair is given by the gauge equivalent pair $\partial L \partial^{-1}=\partial u^{3} \partial^{2}+\partial^{2} u^{3} \partial+\partial v+v \partial, \partial P_{\geq 2}(L) \partial^{-1}=\partial u^{3} \partial^{2}+\partial^{2} u^{3} \partial$. With the additional reduction $v=\lambda_{1} x+\lambda_{2}\left(\lambda_{1,2}=\right.$ const $), \quad w=\lambda=0$, equation (3.94) becomes linear. However, passing to the next nontrivial flow admitting the Kupershmidt constraint, one finds a nontrivial equation corresponding to this reduction. By a shift of the $x$ coordinate we may absorb $\lambda_{2}$ into $x$, that is, we assume $\lambda_{2}=0$. The resulting Lax pair is

$$
\begin{aligned}
L= & u^{3} \partial^{3}+\partial u^{3} \partial^{2}+2 \lambda_{1} x \partial-\lambda_{1} \\
P_{\geq 2}\left(L^{\frac{5}{3}}\right)= & \frac{1}{6\left(2^{\frac{1}{3}}\right)}\left(24 u^{5} \partial^{5}+180 u^{4} u_{x} \partial^{4}+\left(290 u^{3} u_{x}^{2}+140 u^{4} u_{x x}\right) \partial^{3}\right. \\
& +\left(40 u^{4} u_{x x x}+210 u^{3} u_{x} u_{x x}+75 u^{2} u_{x}^{3}\right) \partial^{2}+40 \lambda_{1} x\left(u^{2} \partial^{3}+u u_{x} \partial^{2}\right) \\
& \left.+20 \lambda_{1} u^{2} \partial^{2}\right)
\end{aligned}
$$

leading to the scalar equation

$$
\begin{aligned}
& 0=9\left(2^{\frac{1}{3}}\right) u_{t_{s}}+4 u^{5} u_{x x x x x}+20 u^{4} u_{x} u_{x x x x}+10 u^{4} u_{x x} u_{x x x}+15 u^{3} u_{x}^{2} u_{x x x} \\
& +\left(20 u^{2} u_{x x x}+15 u_{x}^{3}-30 u u_{x} u_{x x}\right) x \lambda_{1}+\left(30 u^{2} u_{x x}-15 u u_{x}^{2}\right) \lambda_{1}+40\left(x u_{x}-u\right)_{u}^{\frac{x}{u}} \lambda_{1}^{2}
\end{aligned}
$$

It represents the reduction $v=\lambda_{1} x, w=0$ of the $t_{5}$-flow of the hierarchy of (3.93).

For 4 th order Lax operators $L=u_{4} \partial^{4}+u_{3} \partial^{3}+u_{2} \partial^{2}+u_{1} \partial+u_{0}+\partial^{-1} u_{-1}+$ $\partial^{-2} u_{-2}$ the constraint (3.63) leads to the conditions $u_{3}=u_{4 x}, u_{1}=0$, 
$u_{-1}=-u_{0 x}$. With $u_{4}=u^{4}, u_{2}=v, u_{0}=w, u_{-2}=z$ one finds

$$
L=\partial u^{4} \partial^{3}+v \partial^{2}+\partial^{-1} w \partial+\partial^{-2} z, \partial^{-1} z, P_{\geq 2}\left(L^{\frac{3}{4}}\right)=\frac{1}{2}\left(u^{3} \partial^{3}+\partial u^{3} \partial^{2}\right)
$$

leading to

$$
4\left(\begin{array}{c}
u \\
v \\
w \\
z
\end{array}\right)_{t_{3}}=\left(\begin{array}{l}
-5 u^{3} u_{x x x}-18 u^{2} u_{x} u_{x x}+3 v_{x}-6 u^{-1} u_{x} v \\
4 u^{3} v_{x x x}-2\left(u^{3}\right)_{x} v_{x x}+12 u^{3} w_{x}-2 u^{4}\left(u^{3}\right)_{x x x x x}-2\left(u^{4}\right)_{x}\left(u^{3}\right)_{x x x x} \\
4 u^{3} w_{x x x}+6\left(u^{3}\right)_{x} w_{x x}+2\left(u^{3}\right)_{x x} w_{x}+12 u^{3} z_{x}+8\left(u^{3}\right)_{x} z \\
4 u^{3} z_{x x x}+10\left(u^{3}\right)_{x} z_{x x}+8\left(u^{3}\right)_{x x} z_{x}+2\left(u^{3}\right)_{x x x} z
\end{array}\right)
$$

Intersecting with the additional reductions of (3.6), we may impose the constraints $z=0$ and $(z=0, w=$ const $)$ on this equation and its hierarchy.

For 5 th order Lax operators $L=u_{5} \partial^{5}+u_{4} \partial^{4}+u_{3} \partial^{3}+u_{2} \partial^{2}+u_{1} \partial+u_{0}+\partial^{-1} u_{-1}$ $+\partial^{-2} u_{-2}$ the Kupershmidt constraint leads to the conditions $u_{4}=\frac{3}{2} u_{5 x}$, $u_{2}=-\frac{1}{4} u_{5 x x x}+\frac{1}{2} u_{3 x}, u_{0}=-\frac{1}{2} u_{1 x}, u_{-2}=\frac{1}{4} u_{1 x x x}-\frac{1}{2} u_{-1 x}$. With $u_{5}=2 u^{5}, u_{3}=$ $\frac{3}{2} u_{5 x x}+2 v, u_{1}=2 w, u_{-1}=w_{x x}+2 z$ one finds

$$
\begin{gathered}
L=u^{5} \partial^{5}+\partial^{3} u^{5} \partial^{2}+v \partial^{3}+\partial v \partial^{2}+w \partial+\partial^{-1} w \partial^{2}+\partial^{-1} z+\partial^{-2} z \partial, \\
P_{\geq 2}\left(L^{\frac{3}{5}}\right)=\left(2^{-\frac{2}{5}}\right)\left(u^{3} \partial^{3}+\partial u^{3} \partial^{2}\right),
\end{gathered}
$$

leading to

$$
\begin{aligned}
\left(2^{\frac{2}{5}}\right) u_{t_{3}}= & 2 u^{3} u_{x x x}+45 u^{2} u_{x} u_{x x}+\frac{6}{5} u^{-1} v_{x}-\frac{18}{5} u^{-2} u_{x} v \\
\left(2^{\frac{2}{5}}\right) v_{t_{3}}=-4 u^{3} v_{x x x} & -7\left(u^{3}\right)_{x} v_{x x}+7\left(u^{3}\right)_{x x} v_{x}+4\left(u^{3}\right)_{x x x} v+6 u^{3} w_{x}-2\left(u^{3}\right)_{x} w \\
& -2 u^{5}\left(u^{3}\right)_{x x x x x}-19\left(u^{5}\right)_{x}\left(u^{3}\right)_{x x x x}-27\left(u^{5}\right)_{x x}\left(u^{3}\right)_{x x x} \\
& +3\left(u^{5}\right)_{x x x}\left(u^{3}\right)_{x x}+6\left(u^{5}\right)_{x x x x}\left(u^{3}\right)_{x}
\end{aligned}
$$




$$
\begin{aligned}
& \left(2^{\frac{2}{5}}\right) w_{t_{3}}=2 u^{3} w_{x x x}+\left(u^{3}\right)_{x} w_{x x}+6 u^{3} z_{x}+2\left(u^{3}\right)_{x} z \\
& \left(2^{\frac{2}{5}}\right) z_{t_{3}}=2 u^{3} z_{x x x}+3\left(u^{3}\right)_{x} z_{x x}+\left(u^{3}\right)_{x x} z_{x} .
\end{aligned}
$$

Admissible reductions to Lax operators of the type (3.6) are given by the constraints $z=$ const and $\left(z=0, w=\lambda_{1} x+\lambda_{2}\right)$.

\section{§3.4. The Hamiltonian Concept}

As discussed in Section 2 the equations (2.16) originate from a Hamiltonian concept making use of the algebraic properties of the $r$-matrix. Hence, the Lax equations (2.16) as evolutionary systems on the algebra $g$ are Hamiltonian. In fact, they are multi-Hamiltonian according to the representations (2.12) involving the three natural brackets (2.8) associated with the $r$-matrices

$$
r_{k}=P_{\geq k}-P_{<k}, \quad k=0,1,2
$$

under consideration. As already mentioned before, it must be pointed out, however, that only for the case $k=0$ all three brackets are in fact Poisson brackets. For $k=1$ and $k=2$ only the linear and the cubic brackets are Poisson brackets, as $r_{1}$ and $r_{2}$ fail to have the additional algebraic properties required to turn the quadratic bracket into a Poisson structure. Both the linear and cubic brackets will be Poisson structures for any $r$-matrix satisfying the modified Yang-Baxter equation (2.4). As (3.101) stem from Lie algebra decompositions of the algebra of pseudo-differential symbols, they do indeed satisfy the Yang-Baxter condition and hence lead to two Hamiltonian formulations (2.12) involving $\mathscr{P}_{1}$ and $\mathscr{P}_{3}$. It was pointed out in Refs. $[18,19]$ that the quadratic bracket is more delicate. A sufficient condition for this bracket to be a Poisson structure, too, involves the skew-symmetric part $\frac{1}{2}\left(r-r^{*}\right)$ of the $r$-matrix. In particular, if this skew-symmetric part agains satisfies the modified Yang-Baxter equation, then this will guarantee the Poisson properties of the quadratic bracket. For the case $k=0$ the $r$-matrix $r_{0}$ turns out to be skew-symmetric. Hence its skew-symmetric part coincides with $r_{0}$, thus satisfies the Yang-Baxter relation and leads to the quadratic Poisson structure. For the cases $k=1$ and $k=2$ the $r$-matrices are not skew-symmetric any more. In fact, it can be shown, that in these cases the 
quadratic bracket does not define a Poisson bracket for generic points in the algebra. Further, from the deformation property $(2.10)$ it is clear that the linear and the cubic brackets are compatible if and only if the quadratic bracket is a Poisson structure. Hence, on the general abstract level, for the cases $k=1$ and $k=2$ the Poisson brackets associated with $\mathscr{P}_{1}$ and $\mathscr{P}_{2}$ are not compatible.

As we will see in this section the general multi-Hamiltonian formulation (2.12) for the Lax operators leads to Hamiltonian formulations of the nonlinear integrable equations discussed before. As a surprising fact, also the abstract quadratic bracket for the cases $k=1$ and $k=2$ will yield a Hamiltonian formulation for the corresponding equations. This is related to the fact that the nonlinear equations of interest are related to Lax operators of a specific form. In particular, all the equations discussed before are connected with operators of the form (3.4) or (3.5), (3.6). Hence, we are not dealing with Lax operators in general position, but we are concerned with the restriction of the general flows (2.12) to subspaces of the form (3.4), (3.5), (3.6). These subspaces are invariant with respect to the particular Hamiltonian dynamics associated with the Casimir functions on $g$. However, these subspaces will not automatically be invariant subspaces for the brackets under consideration. Special care has to be taken in evaluating the general Hamiltonian structures for some of the Lax operators used in the last section. If these operators form a proper Poisson subspace of the abstract Hamiltonian structure, then simple restriction of the general Poisson bracket to this subspace provides the Hamiltonian formulation for the associated nonlinear equations. In some cases, however, the general Poisson structure can not be properly restricted to the class of Lax operators under consideration. In such a situation it is often very useful to embed this class into a larger space of operators, which forms a proper Poisson subspace and hence enjoys a restricted Hamiltonian structure. Then Dirac reduction can be invoked to reduce (rather than restrict) the Poisson bracket to the class of operators under consideration. A formulation of this reduction process in suitable notation was given in Ref. [19]. It may be summarized as follows:

\section{Dirac reduction.}

For two linear spaces $U$ and $C$, spanned by the coordinates $u \in U$ and $c \in C$, respectively, let 


$$
\mathscr{P}(u, c)=\left(\begin{array}{cc}
\mathscr{P}_{u u}(u, c) & \mathscr{P}_{u c}(u, c) \\
\mathscr{P}_{c u}(u, c) & \mathscr{P}_{c c}(u, c)
\end{array}\right)
$$

be a Poisson tensor on $U \oplus C$. Then, for arbitrary fixed $c \in C$, the tensor

$$
\Theta(u ; c)=\mathscr{P}_{u u}(u, c)-\mathscr{P}_{u c}(u, c)\left(\mathscr{P}_{c c}(u, c)\right)^{-1} \mathscr{P}_{c u}(u, c)
$$

is a Poisson tensor on the affine space $c+U$ spanned by the variable $u$.

The Dirac reduced tensor $\Theta$ depends on the fixed $c$ as a parameter only. It will turn out that many of the Hamiltonian operators connected to the equations of Section 3.2 originate from this reduction process. The inverse $\mathscr{P}_{c c}^{-1}$ in (3.103) then accounts for the non-localities (inverses of differential operators) often encountered in such formal Poisson brackets. We point out that this reduction depends on a rather severe "technicality": the element $\mathscr{P}_{c c}$ of the Hamiltonian matrix (3.102) has to be invertible. Hence, in some reductions there will not be any chance to obtain a Poisson structure, when $\mathscr{P}_{c c}$ is degenerate. In fact, some of the equations of Section 3.2 (such as Burgers equation (3.17)) are of dissipative nature and do not admit a Hamiltonian formulation. As we will see, the general Poisson brackets can not be properly restricted to the Lax operators of those cases, and Dirac reduction cannot be invoked for the reason given above. Hence, we may loose the Hamiltonian structure in the reduction process from generic $L \in g$ to specific Lax operators of the form (3.4), (3.5), (3.6).

On the other hand, it can happen that the Dirac reduced tensor (3.103) is a Poisson structure, even if the original tensor (3.102) was not a Poisson tensor. This seems to be the situation that we will encounter in context of the quadratic bracket, which is not a Poisson bracket for $k=1$ and $k=2$. It will turn out that in these cases Dirac reduction does indeed lead to Hamiltonian formulations, although the general bracket does not enjoy any distinguished algebraic properties.

For a detailed discussion of the reduction properties of the tensors (2.8) we first rewrite them in terms of the particular $r$-matrices (3.101) under consideration here. The transpose $r_{k}^{*}$ entering the definitions are given by $r_{k}^{*}=P_{\geq k}^{*}-P_{<k}^{*}$, where $P_{\geq k}^{*}$ and $P_{<k}^{*}$ are the projections to the dual subalgebras given by the dual decomposition $g^{*}=g=g_{\geq k}^{*} \oplus g_{<k}^{*}$ with

$$
g_{<k}^{*}=\left\{\sum_{i \geq-k} \partial^{i} u_{i}\right\}, \quad g_{\geq k}^{*}=\left\{\sum_{i<-k} \partial^{i} u_{i}\right\}
$$


This dual decomposition is readily verified observing $\left\langle g_{<k}^{*}, g_{\geq k}\right\rangle=$ $<g_{\geq k}^{*}, g_{<k}>=0$ using the trace duality $(2.1) /(2.21)$. Thus, the tensors are defined in terms of the four projections $P_{\geq k}, P_{<k}, P_{\geq k}^{*}$ and $P_{<k}^{*}$. Using $P_{\geq k}+P_{<k}=P_{\geq k}^{*}+P_{<k}^{*}=1$ we can either eliminate $P_{<k}=1-P_{\geq k}, P_{\geq k}^{*}=1-P_{<k}^{*}$ or $P_{\geq k}=1-P_{<k}, P_{<k}^{*}=1-P_{\geq k}^{*}$ and obtain two equivalent representations for each of the tensors (2.8):

$$
\begin{aligned}
\mathscr{P}_{1}(L) \nabla H= & 2\left[P_{\geq k}(\nabla H), L\right]-2 P_{<k}^{*}([\nabla H, L]) \\
= & -2\left[P_{<k}(\nabla H), L\right]+2 P_{\geq k}^{*}([\nabla H, L]) \\
\mathscr{P}_{2}(L) \nabla H= & 2\left[P_{\geq k}(L \nabla H+\nabla H L), L\right]-2 L P_{<k}^{*}([\nabla H, L])-2 P_{<k}^{*}([\nabla H, L]) L \\
= & -2\left[P_{<k}(L \nabla H+\nabla H L), L\right]+2 L P_{\geq k}^{*}([\nabla H, L])+2 P_{\geq k}^{*}([\nabla H, L]) L \\
\mathscr{P}_{3}(L) \nabla H= & 2\left[P_{\geq k}(L \nabla H L), L\right]-2 L P_{<k}^{*}([\nabla H, L]) L \\
= & -2\left[P_{<k}(L \nabla H L), L\right]+2 L P_{\geq k}^{*}([\nabla H, L]) L
\end{aligned}
$$

It turns out that for each tensor the second representation yields direct access to the highest differential order of the operators $\mathscr{P}_{i}(L) \nabla H, i=1,2,3$, whereas the first representation yields information about the lowest orders present. To see this, we summarize the projections entering (3.105). At this stage it is more convenient to parametrize each element $A \in g$ by

$$
A=\sum_{i \geq 0}^{N} a_{i} \partial^{i}+\sum_{i<0} \partial^{i} a_{i}
$$

Thus, the projections are given by

$$
\begin{aligned}
& \underline{k=0:} \quad P_{\geq 0}(A)=P_{<0}^{*}(A)=a_{N} \partial^{N}+. .+a_{1} \partial+a_{0}, \\
& P_{<0}(A)=P_{\geq 0}^{*}(A)=\partial^{-1} a_{-1}+\partial^{-2} a_{-2}+\cdots, \\
& \text { k=1: } \quad P_{\geq 1}(A)=a_{N} \partial^{N}+. .+a_{2} \partial^{2}+a_{1} \partial \text {, } \\
& P_{<1}^{*}(A)=a_{N} \partial^{N}+. .+a_{2} \partial^{2}+a_{1} \partial+a_{0}+\partial^{-1} a_{-1}, \\
& P_{<1}(A)=a_{0}+\partial^{-1} a_{-1}+\partial^{-2} a_{-2}+\cdots, \\
& P_{\geq 1}^{*}(A)=\partial^{-2} a_{-2}+\partial^{-3} a_{-3}+\cdots, \\
& \underline{k=2:} \quad P_{\geq 2}(A)=a_{N} \partial^{N}+. .+a_{3} \partial^{3}+a_{2} \partial^{2} \text {, } \\
& P_{<2}^{*}(A)=a_{N} \partial^{N}+. .+a_{2} \partial^{2}+a_{1} \partial+a_{0}+\partial^{-1} a_{-1}+\partial^{-2} a_{-2} \text {, } \\
& P_{<2}(A)=a_{1} \partial+a_{0}+\partial^{-1} a_{-1}+\cdots, \\
& P_{\geq 2}^{*}(A)=\partial^{-3} a_{-3}+\partial^{-4} a_{-4}+\cdots \text {. }
\end{aligned}
$$


The case $k=0$.

We will be interested in operators $L \in g_{<0}^{*}=g_{\geq 0}$ of the form

$$
L=c_{N} \partial^{N}+c_{N-1} \partial^{N-1}+u_{N-2} \partial^{N-2}+. .+u_{1} \partial+u_{0}
$$

with fixed $c_{N}, c_{N-1}$ and dynamical fields $u_{N-2}, . ., u_{0}$. These are the Gelfand-Dikii spectral problems, for which some of the isospectral equations were exhibited in Section 3.2 for $N \leq 5$. Inserting such an operator into (3.105), it becomes clear from the first representation for the linear tensor that $\mathscr{P}_{1}^{(k=0)}(L) \nabla H$ will be a purely differential operator. From the second representation it is clearly seen that the highest differential order stems from the commutator of $P_{<0}(\nabla H)$ with $L$. This highest order will be at most $N-2$. Hence, for $L$ of the form (3.108) with given $c_{N}$ and $c_{N-1}$, the Hamiltonian vectorfield $\mathscr{P}_{1}^{(k=0)}(L) \nabla H$ is tangent to the affine spaces of operators (3.108) spanned by the coordinates $u_{N-2}, . ., u_{0}$. As a result, these spaces are proper Poisson subspaces of the linear bracket on $g$. Hence, the reduction of the integrable hierarchy $(2.12)$ to operators of the form $(3.4, k=0)=(3.108)$ is in fact a proper Hamiltonian restriction. The corresponding equations inherit a Hamiltonian structure from the linear bracket on $g$.

The quadratic tensor defines a Poisson bracket on $g$, as the underlying $r$-matrix is skew symmetric for the present case $k=0$. From the first representation in (3.105) it is clear that $\mathscr{P}_{2}^{(k=0)}$ yields a purely differential operator, if $L$ is a purely differential operator. Hence, the quadratic bracket can be properly restricted to the subalgebra $g_{\geq 0}$ of differential operators. Inserting an operator $L$ of the form (3.108) into the second representation in (3.105), one sees that the highest differential order of the Hamiltonian vectorfield $\mathscr{P}_{1}^{(k=0)}(L) \nabla H$ will be $N-1$. Hence, operators of the form (3.108) spanned by the variables $u_{N-2}, . ., u_{0}$ do not form a proper Poisson subspace. Instead, the quadratic bracket can be properly restricted to affine spaces of the form

$$
L=c_{N} \partial^{N}+u_{N-1} \partial^{N-1}+u_{N-1} \partial^{N-2}+. .+u_{1} \partial+u_{0}
$$

spanned by the coordinates $u_{N-1}, \ldots, u_{0}$ (with $c_{N}$ given and fixed). As the spaces (3.108) sit inside (3.109), it is simple to invoke Dirac reduction to reduce the quadratic bracket on (3.109) by the constraint $u_{N-1}=c_{N-1}$. 
For the cubic tensor the first representation in (3.105) shows that $\mathscr{P}_{3}^{(k=0)}$ yields a purely differential operator, if $L$ is a purely differential operator. Hence, also the cubic bracket can be properly restricted to the subalgebra $g_{\geq 0}$ of differential operators. Inserting an operator $L$ of the form (3.108) into the second representation in (3.105), one sees that the highest differential order of the Hamiltonian vectorfield $\mathscr{P}_{1}^{(k=0)}(L) \nabla H$ will be $2 N-1$. Hence, operators of the form (3.108) spanned by the variables $u_{N-2}, . ., u_{0}$ do not form a proper Poisson subspace. In fact, there are no obvious proper Poisson subspaces for the cubic bracket, apart from the trivial case of first order operators. Nevertheless, Dirac reduction can be invoked to restrict the cubic bracket on the differential operators to the affine subspaces of the form (3.108).

It was demonstrated in [19] that these considerations lead to the usual bi-Hamiltonian formulations for the isospectral hierarchies associated with the Lax operators of the type (3.108). The two basic Hamiltonian operators $\Theta_{1}$ and $\Theta_{2}$, say, are obtained from the linear and the quadratic bracket. The reduction of the cubic bracket turns out to be given by $\Theta_{3}=\frac{1}{4} \Theta_{2} \Theta_{1}^{-1} \Theta_{2}$, involving the recursion operator $\Theta_{2} \Theta_{1}^{-1}$ of the bi-Hamiltonian formulation given by $\Theta_{1}$ and $\Theta_{2}$.

The case $k=1$.

We will be interested in operators $L \in g_{<1}^{*}$ of the form

$$
L=c_{N} \partial^{N}+u_{N-1} \partial^{N-1}+. .+u_{1} \partial+u_{0}+\partial^{-1} u_{-1}
$$

with fixed $c_{N}$ and dynamical fields $u_{N-1}, . ., u_{-1}$. These are the spectral problems, for which some of the isospectral equations were exhibited in Section 3.2 for $N \leq 5$. Inserting such an operator into (3.105), it becomes clear from the first representation for the linear tensor that $\mathscr{P}_{1}^{(k=1)}(L) \nabla H$ will be of the form $D_{1}+\partial^{-1} D_{2}$, where $D_{1,2}$ are differential operators. It is easy to see that such operators do again lie in $g_{<1}^{*}$. From the second representation it is clearly seen that the highest differential order.stems from the commutator of $P_{<1}(\nabla H)$ with $L$. This highest order will be at most $N-1$. Hence, for $L$ of the form (3.110) with given $c_{N}$, the Hamiltonian vectorfield $\mathscr{P}_{1}^{(k=1)}(L) \nabla H$ is tangent to the affine spaces of operators (3.110) spanned by the coordinates $u_{N-1}, . ., u_{-1}$. As a result, these spaces are proper Poisson subspaces of the 
linear bracket on $g$. Hence, the reduction of the integrable hierarchy (2.12) to operators of the form $(3.4, k=1)=(3.110)$ is in fact a proper Hamiltonian restriction. The corresponding equations inherit a Hamiltonian structure from the linear bracket on $g$.

We now calculate this Poisson structure for $N \leq 4$. We insert $L=c_{4} \partial^{4}+u_{3} \partial^{3}+u_{2} \partial^{2}+u_{1} \partial+u_{0}+\partial^{-1} u_{-1}$ and (according to (2.22))

$$
\nabla H=\frac{\delta H}{\delta u_{-1}}+\partial^{-1} \frac{\delta H}{\delta u_{0}}+\partial^{-2} \frac{\delta H}{\delta u_{1}}+\partial^{-3} \frac{\delta H}{\delta u_{2}}+\partial^{-4} \frac{\delta H}{\delta u_{3}}
$$

into (3.105). Thus, the Hamiltonian equation $\frac{d}{d t} L=\mathscr{P}_{1}^{(k=1)}(L) \nabla H$ translates to

$$
\frac{d}{d t}\left(\begin{array}{l}
u_{-1} \\
u_{0} \\
u_{1} \\
u_{2} \\
u_{3}
\end{array}\right)=2 \Theta \nabla H=2\left(\begin{array}{ccccc}
0 & \Theta_{-10} \Theta_{-11} \Theta_{-12} \Theta_{-13} \\
\Theta_{0-1} & \Theta_{00} & \Theta_{01} & \Theta_{02} & 0 \\
\Theta_{1-1} & \Theta_{10} & \Theta_{11} & 0 & 0 \\
\Theta_{2-1} & \Theta_{20} & 0 & 0 & 0 \\
\Theta_{3-1} & 0 & 0 & 0 & 0
\end{array}\right)\left(\begin{array}{l}
\delta H / \delta u_{-1} \\
\delta H / \delta u_{0} \\
\delta H / \delta u_{1} \\
\delta H / \delta u_{2} \\
\delta H / \delta u_{3}
\end{array}\right),
$$

where the non-zero elements are given by

$$
\begin{aligned}
& \Theta_{0-1}=u_{1} D+u_{2} D^{2}+u_{3} D^{3}+c_{4} D^{4}, \Theta_{1-1}=2 u_{2} D+3 u_{3} D^{2}+4 c_{4} D^{4}, \\
& \Theta_{2-1}=3 u_{3} D+6 c_{4} D^{2}, \Theta_{3-1}=4 c_{4} D \\
& \Theta_{00}=D u_{2}+u_{2} D+u_{3} D^{2}-D^{2} u_{3}+D^{3} c_{4}+c_{4} D^{3} \\
& \Theta_{10}=D u_{3}+2 u_{3} D+3 c_{4} D^{2}-D^{2} c_{4} \\
& \Theta_{20}=D c_{4}+3 c_{4} D, \Theta_{11}=2 D c_{4}+2 c_{4} D
\end{aligned}
$$

and skew symmetry of $\Theta$. Here, $D$ is the differential operator acting on the dynamical fields, which we wish to distinguish from the (pseudo-) differential symbol $\partial$ (acting on a suitable space of test functions). Now, the linear Poisson tensors for the various equations of Section $3.2, k=1$, are obtained easily. For instance, for $L=\partial+u+\partial^{-1} v$ we can restrict (3.112) to the smaller subspace given by $u_{1}=1, u_{2}=u_{3}=c_{4}=0$. Putting $u_{-1}=v$ and $u_{0}=u$ one obtains

$$
\frac{d}{d t}\left(\begin{array}{l}
u \\
v
\end{array}\right)=2\left(\begin{array}{ll}
0 & D \\
D & 0
\end{array}\right)\left(\begin{array}{l}
\delta H / \delta u \\
\delta H / \delta v
\end{array}\right)
$$


as the "first" Hamiltonian formulation for the Kaup-Broer system (3.16). As this equation is obtained from $\frac{d}{d t_{2}} \mathbb{L}=\left[P_{\geq 1}\left(\mathbb{L}^{2}\right), \mathbb{L}\right]=\mathscr{P}_{1}^{(k=1)}(L) \nabla \operatorname{tr}\left(\mathbb{L}^{3}\right) / 6$, the Hamiltonian function for (3.16) is calculated as $H=\operatorname{tr}\left(\mathbb{L}^{3}\right) / 6=\frac{1}{2} \int\left(u_{x} v+v^{2}+\right.$ $\left.u^{2} v\right) d x$ using (2.21). We had observed in Section 3.2 that the Kaup-Broer system can be restricted to Burgers equation (3.17) by the constraint $u_{-1}=v=0$. However, as is clearly seen from (3.112), the linear bracket cannot be properly restricted to the subspace given by this constraint. Further, Dirac reduction cannot be invoked, as the tensor element $\Theta_{-1-1}$ vanishes. Hence, although Burgers equation is a restriction of the Hamiltonian equation (3.16), the Hamiltonian structure is lost in the reduction. Also, the Hamiltonian function $H=\operatorname{tr}\left(\mathbb{L}^{3}\right) / 6$ becomes trivial for $v=0$.

In the same way we can obtain the Hamiltonian formulations associated with higher Lax operators. For $N=2$ we consider $L=\partial^{2}+u \partial+v+\partial^{-1} w$. Putting $u_{-1}=w, u_{0}=v, u_{1}=u, u_{2}=1, u_{3}=c_{4}=0$, the Hamiltonian equation (3.112) translates to

$$
\left(\begin{array}{c}
\frac{d}{d t}^{u} \\
\\
w
\end{array}\right)=2\left(\begin{array}{ccc}
0 & 0 & 2 D \\
0 & 2 D & u D+D^{2} \\
2 D & D u-D^{2} & 0
\end{array}\right)\left(\begin{array}{l}
\delta H / \delta u \\
\delta H / \delta v \\
\delta H / \delta w
\end{array}\right)
$$

This is the Hamiltonian formulation for the equations (3.19) and (3.23). The Hamiltonian functions can be calculated as $H=\operatorname{tr}\left(L^{2}\right) / 4$ for (3.19) and $H=\operatorname{tr}\left(L^{\frac{5}{2}}\right) / 5$ for (3.23), e.g. one finds $\operatorname{tr}\left(L^{2}\right) / 4=\frac{1}{2} \int v w d x$. Again, in the reduction to $w=0$ we will loose the Hamiltonian structure for equation (3.20). However, in the reduction ( $w=0, v=\lambda=$ const) to the modified $\mathbb{K d V}$ equation (3.24) we can use Dirac reduction on (3.115) to obtain

$$
\frac{d}{d t} u=-2(0 ; 2 D)\left(\begin{array}{cc}
2 D & u D+D^{2} \\
D u-D^{2} & 0
\end{array}\right)^{-1}\left(\begin{array}{c}
0 \\
2 D
\end{array}\right) \frac{\delta H}{\delta u}
$$

as a formal Hamiltonian formulation. With

$\left(\begin{array}{cc}2 D & u D+D^{2} \\ D u-D^{2} & 0\end{array}\right)^{-1}=\left(\begin{array}{cc}0 & (u-D)^{-1} D^{-1} \\ D^{-1}(u+D)^{-1} & -2 D^{-1}(u+D)^{-1} D(u-D)^{-1} D^{-1}\end{array}\right)$ 
one obtains a formal Hamiltonian formulation

$$
\frac{d u}{d t}=16(u+D)^{-1} D(u-D)^{-1} \frac{\delta H}{\delta u}
$$

for the modified $\mathrm{KdV}$ equation (3.24). This, in fact, corresponds to the formal Hamiltonian operator $R^{-1} \Theta_{1}=\Theta_{1} \Theta_{2}^{-1} \Theta_{1}$ obtained from the recursion operator $R=\Theta_{2} \Theta_{1}^{-1}$. Here $\Theta_{1}=D$ and $\Theta_{2}=D^{3}-D u D^{-1} u D=-D(u-D)$. $D^{-1}(u+D) D$ are the two Hamiltonain operators constituting the well known bi-Hamiltonian formulation for the modified $\mathrm{KdV}$. We will identify these operators as reductions of the quadratic and the cubic bracket, soon.

In a similar fashion, all Hamiltonian formulations associated with any Lax operator of the form (3.110) can be calculated for any $N$. In all cases one looses the Hamiltonian structure in the reduction to $u_{-1}=0$. However, Dirac reduction will lead to the Hamiltonian operators for the equations associated with the reduction $\left(u_{-1}=0, u_{0}=\lambda=\right.$ const $)$.

The quadratic tensor $\mathscr{P}_{2}$ in (2.8) does not define a Poisson bracket on $g$ for the present choice $k=1$. Nevertheless, it turns out that Hamiltonian formulations are hidden in this tensor for certain reductions. Considering the reduction properties we first look at the second representation for $\mathscr{P}_{2}$ in (3.105). Inserting $L$ of the form (3.110), the highest differential order of the operator $\mathscr{P}_{2}^{(k=1)}(L) \nabla H$ is easily seen to be at most $N-1$, matching the form of the highest differential orders of the ansatz (3.110). Concerning the lowest differential orders we insert $L$ of the form (3.110) into the first representation for $P_{2}$ in (3.105). The second and third term will produce expressions of the form $-2\left(u_{0}+\partial^{-1} u_{-1}\right) \partial^{-1} a_{-1}$ and $-2 \partial^{-1} a_{-1} \partial^{-1} u_{-1}$, where $a_{-1}$ is the corresponding coefficient of the operator $[\nabla H, L]$. Such terms do not lie in the dual subalgebra $g_{<1}^{*}$, from which the Lax operators (3.110) were chosen. Hence, the quadratic bracket cannot be properly restricted to $g_{<1}^{*}$. However, choosing $L$ to be of the form

$$
L=c_{N} \partial^{N}+u_{N-1} \partial^{N-1}+. .+u_{1} \partial+\lambda, \quad N \geq 2,
$$

the operator $\mathscr{P}_{2}^{(k=1)}(L) \nabla H$ will take values in $g_{<1}^{*}$. Still, expressions of both zero and minus first order, i.e. of the form $\partial^{-1}(.$.$) , will be present. Hence,$ the quadratic bracket cannot be properly restricted to operators of the form (3.119), either. Nevertheless, these properties allow to apply Dirac reduction 
to operators (3.119) in a simple way. We demonstrate this considering the Lax operator $L=\partial^{2}+u \partial$ associated with the modified $\mathrm{KdV}$ hierarchy. We think of $L$ as $L=\partial^{2}+u \partial+v+\partial^{-1} w$, subject to the constraint $v=w=0$. Inserting $\nabla H=\frac{\delta H}{\delta w}+\partial^{-1} \frac{\delta H}{\delta v}+\partial^{-2} \frac{\delta H}{\delta u}$, the evolution equation $\frac{d}{d t} L=\mathscr{P}_{2}^{(k=1)}(L) \nabla H$ translates into

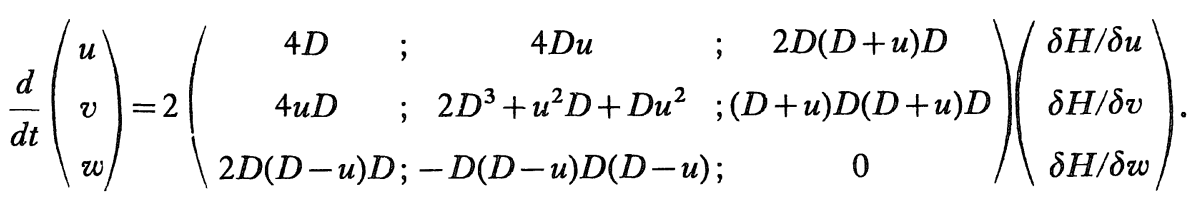

The Dirac reduced equations reads

$$
\frac{d}{d t} u=2(4 D-\Delta) \frac{\delta H}{\delta u}
$$

where

$$
\begin{aligned}
\Delta=(4 D u ; 2 D(D+u) D) & \left(\begin{array}{ccc}
2 D^{3}+u^{2} D+D u^{2} & ;(D+u) D(D+u) D \\
-D(D-u) D(D-u) & ; & 0
\end{array}\right)^{-1} \\
& \cdot\left(\begin{array}{c}
4 u D \\
2 D(D-u) D
\end{array}\right) .
\end{aligned}
$$

Making use of the factorizations of the operators, the formal inverse in (3.122) can be evaluated, leading to

$$
\frac{d}{d t} u=-8 D \frac{\delta H}{\delta u}
$$

Despite the fact that the general quadratic bracket is not a Poisson structure, its Dirac reduction leads to the well known "first" Hamiltonian formulation of the modified $\mathrm{KdV}$ given by the differential operator.

In a similar way the Dirac reduction of the quadratic bracket can be performed for any operator of the form (3.119). We expect that Hamiltonian formulations for the isospectral hierarchies for these operators can be obtained this way for $N>2$, ton. However, as the general quadratic tensor does not 
enjoy any Poisson properties, there is no structural justification for this construction and the derivation of Hamiltonian formulations such as (3.123) from the abstract bracket remains somewhat mysterious.

The cubic tensor in (2.8) does define a Poisson bracket, so that the following considerations are well motivated. An analysis similar to the case of the quadratic bracket shows that only for Lax operators of the restricted class (3.119) in $g_{\geq 1}$ the operator $\mathscr{P}_{3}^{(k=1)}(L) \nabla H$ will take values in $g_{<1}^{*}$. However, for the cubic bracket, this Hamiltonian vectorfield will actually take values in $g_{\geq 1} \subset g_{<1}^{*}$, when the parameter $\lambda$ vanishes. Hence, the cubic bracket can be properly restricted to the subalgebra $g_{\geq 1}$. Considering the second representation for $\mathscr{P}_{3}$ in (3.105), one easily deduces that the highest differential order of $\mathscr{P}_{3}^{(k=1)}(L) \nabla H$ will be $2 N-2$. Hence, there only is one simple Poisson subspace for the cubic bracket in the class (3.110), given by second order operators of the form $L=v \partial^{2}+u \partial$. We insert $\nabla H=\partial^{-} \frac{\delta H}{\delta v}+\partial^{-3} \frac{\delta H}{\delta v}$ and $\frac{d}{d t} L=\mathscr{P}_{3}^{(k=1)}(L) \nabla H$ translates to

$\frac{d}{d t}\left(\begin{array}{l}u \\ v\end{array}\right)=2\left(\begin{array}{ccc}u v D^{2} v-v D^{2} v u & ; & -v D^{2} v^{2}+D u v^{2}-2 u D v^{2} \\ v^{2} D^{2} v+u v^{2} D-2 v^{2} D u & ; & -v^{3} D-D v^{3}\end{array}\right)\left(\begin{array}{l}\delta H / \delta u \\ \delta H / \delta v\end{array}\right)$.

Dirac reduction to $v=1$ produces the "second" Hamiltonian formulation

$$
d t=\left(-D^{3}+D u D^{-1} u D\right) \frac{\delta H}{\delta u}
$$

of the modified $\mathrm{KdV}$ hierarchy.

For $N>2$ Dirac reduction can be performed to obtain further Hamiltonian formulations of the isospectral hierarchies associated with Lax operators of the form (3.119).

The case $k=2$.

We will be interested in operators $L \in g_{<2}^{*}$ of the form

$$
L=u_{N} \partial^{N}+u_{N-1} \partial^{N-1}+. .+u_{1} \partial+u_{0}+\partial^{-1} u_{-1}+\partial^{-2} u_{-2}
$$


spanned by the fields $u_{N}, . ., u_{-2}$. These are the spectral problems, for which some of the isospectral equations were exhibited in Section 3.2 for $N \leq 5$. Inserting such an operator into (3.105), it becomes clear from the first representation for the linear tensor that $\mathscr{P}_{1}^{(k=1)}(L) \nabla H$ will be of the form $D_{1}+\partial^{-1} D_{2}+\partial^{-2} D_{3}$, where $D_{1,2,3}$ are differential operators. It is easy to see that such operators do again lie in $g_{<2}^{*}$. From the second representation it is clearly seen that the highest differential order stems from the commutator of $P_{<2}(\nabla H)$ with $L$. This highest order will be at most $N$. Hence, for $L$ of the form (3.126), the Hamiltonian vectorfield $\mathscr{P}_{1}^{(k=2)}(\mathbb{L}) \nabla H$ is tangent to the spaces of operators (3.126). As a result, the spaces given by these operators are proper Poisson subspaces of the linear bracket on $g$. Hence, the reduction of the integrable hierarchy (2.12) to operators of the form $(3.4, k=2)=(3.126)$ is in fact a proper Hamiltonian restriction. The corresponding equations inherit a Hamiltonian structure from the linear bracket on $g$.

We now calculate this Poisson structure for $N \leq 3$. We insert $L=u_{3} \partial^{3}+. .+\partial^{-2} u_{-2}$ and (according to (2.22))

$$
\nabla H=\frac{\delta H}{\delta u_{-2}} \partial+\frac{\delta H}{\delta u_{-1}}+\partial^{-1} \frac{\delta H}{\delta u_{0}}+\partial^{-2} \frac{\delta H}{\delta u_{1}}+\partial^{-3} \frac{\delta H}{\delta u_{2}}+\partial^{-4} \frac{\delta H}{\delta u_{3}}
$$

into (3.105). Thus, the Hamiltonian equation $\frac{d}{d t} L=\mathscr{P}_{1}^{(k=2)}(L) \nabla H$ translates to

$$
\frac{d}{d t}\left(\begin{array}{l}
u_{-2} \\
u_{-1} \\
u_{0} \\
u_{1} \\
u_{2} \\
u_{3}
\end{array}\right)=2 \Theta \nabla H=2\left(\begin{array}{cccccc}
\Theta_{-2-2} \Theta_{-2-1} \Theta_{-20} \Theta_{-21} \Theta_{-22} \Theta_{-23} \\
\Theta_{-1-2} & 0 & \Theta_{-10} \Theta_{-11} \Theta_{-12} & 0 \\
\Theta_{0-2} & \Theta_{0-1} & \Theta_{00} & \Theta_{01} & 0 & 0 \\
\Theta_{1-2} & \Theta_{1-1} & \Theta_{10} & 0 & 0 & 0 \\
\Theta_{2-2} & \Theta_{2-1} & 0 & 0 & 0 & 0 \\
\Theta_{3-2} & 0 & 0 & 0 & 0 & 0
\end{array}\right)\left(\begin{array}{l}
\delta H / \delta u_{-2} \\
\delta H / \delta u_{-1} \\
\delta H / \delta u_{0} \\
\delta H / \delta u_{1} \\
\delta H / \delta u_{2} \\
\delta H / \delta u_{3}
\end{array}\right),
$$

where the non-zero elements are given by

$$
\begin{aligned}
& \Theta_{-2-2}=-D u_{-2}-u_{-2} D, \Theta_{-1-2}=-D u_{-1}, \Theta_{0-2}=-D u_{0}+u_{0} D, \\
& \Theta_{1-2}=-D u_{1}+2 u_{1} D+u_{2} D^{2}+u_{3} D^{3}, \Theta_{2-2}=-D u_{2}+3 u_{2} D+3 u_{3} D^{2},
\end{aligned}
$$




$$
\begin{aligned}
& \Theta_{3-2}=-D u_{3}+4 u_{3} D, \Theta_{0-1}=u_{1} D+u_{2} D^{2}+u_{3} D^{3}, \Theta_{1-1}=2 u_{2} D+3 u_{3} D^{2}, \\
& \Theta_{2-1}=3 u_{3} D, \Theta_{00}=u_{2} D+D u_{2}+u_{3} D^{2}-D^{2} u_{3}, \Theta_{10}=2 u_{3} D+D u_{3},
\end{aligned}
$$

and skew symmetry of $\Theta$. Now, the linear Poisson tensors for the various equations of Section 3.2, $k=2$, are obtained easily. For instance, for $L=u \partial+v+\partial^{-1} w+\partial^{-2} z$ we put $u_{-2}=z, u_{-1}=w, u_{0}=v, u_{1}=u, u_{2}=u_{3}=0$ and obtain

$$
\frac{d}{d t}\left(\begin{array}{c}
u \\
v \\
w \\
z
\end{array}\right)=2\left(\begin{array}{cccc}
0 & 0 & 0 & -D u+2 u D \\
0 & 0 & u D & -D v+v D \\
0 & D u & 0 & -D w \\
-u D+2 D u & -v D+D v & -w D & -z D-D z
\end{array}\right)\left(\begin{array}{l}
\delta H / \delta u \\
\delta H / \delta v \\
\delta H / \delta w \\
\delta H / \delta z
\end{array}\right)
$$

as the "first" Hamiltonian formulation for the system (3.35). As this equation is obtained from $\frac{d}{d t_{2}} L=\left[P_{\geq 2}\left(L^{2}\right), L\right]=\mathscr{P}_{1}^{(k=2)}(L) \nabla \operatorname{tr}\left(L^{3}\right) / 6$, the Hamiltonian function for (3.35) is calculated as $H=\operatorname{tr}\left(L^{3}\right) / 6=\frac{1}{2} \int\left(u w^{2}+v^{2} w+2 u v z+u v_{x} w+\right.$ $\left.z u u_{x}\right) d x$ using (2.21). We had observed in Section 3.2 that this system can be restricted to the equations (3.36) by the constraints $z=0,(z=0, w=0)$, and $(z=0, w=0, v=\lambda x)$, respectively. As is clearly seen from (3.130), the linear bracket cannot be properly restricted to the subspace given by these constraints.

Further, Dirac reduction cannot be invoked, as the corresponding matrix blocks are degenerate. Hence, although (3.36) are restrictions of the Hamiltonian equation (3.35) the Hamiltonian structure is lost in the reduction. Also, the Hamiltonian function $H=\operatorname{tr}\left(L^{3}\right) / 6$ becomes trivial for these constraints.

In the same way we can obtain the Hamiltonian formulations associated with higher Lax operators. For $N=2$ we consider $L=u^{2} \partial^{2}+v \partial+w+\partial^{-1} z+$ $\partial^{-2} r$. Putting $u_{-2}=r, u_{-1}=z, u_{0}=w, u_{1}=v, u_{2}=u^{2}, u_{3}=c_{4}=0$, the Hamiltonian equation (3.128) translates to 


$$
\begin{gathered}
\frac{d}{d t}\left(\begin{array}{c}
u \\
v \\
w \\
z \\
r
\end{array}\right)=2\left(\begin{array}{cccc}
0 & 0 & 0 & 0 \\
0 & 0 & 0 & 2 u^{2} D \\
0 & 0 & 2 u D u & v D+u^{2} D^{2} \\
0 & 2 D u^{2} & D v-D^{2} u^{2} & 0 \\
u^{-1} D u^{2} & -v D+2 D v-D^{2} u^{2} & -w D+D w & -z D \\
\\
-D v+2 v D+u^{2} D^{2} \\
-D w+w D \\
-D z \\
-D r-r D
\end{array}\right)\left(\begin{array}{c}
\frac{\delta H}{\delta u} \\
\frac{\delta H}{\delta v} \\
\frac{\delta H}{\delta w} \\
\frac{\delta H}{\delta z} \\
\frac{\delta H}{\delta r}
\end{array}\right)
\end{gathered}
$$

This is the Hamiltonian formulation for the equation (3.38). The Hamiltonian function is calculated as $H=\operatorname{tr}\left(L^{2}\right) / 4=\frac{1}{2} \int w z d x$. Again, in the reduction to (3.39) we will loose the Hamiltonian structure and the Hamiltonian function will become trivial.

However, in the reduction $(r=z=0, w=$ const, $v=\lambda x)$ to the Dym equation (3.44) we can use Dirac reduction on (3.131). For $\lambda=0$ one obtains

$$
\frac{d}{d t} u=16\left(u^{2} D u^{2}\right)\left(\frac{1}{u^{3}} D^{-3} \frac{1}{u^{3}}\right)\left(u^{2} D u^{2}\right)\left(\frac{1}{u^{3}} D^{-3} \frac{1}{u^{3}}\right)\left(u^{2} D u^{2}\right) \frac{\delta H}{\delta u}
$$

as a formal Hamiltonian formulation. This, in fact, corresponds to the formal Hamiltonian operator $R^{-2} \Theta_{1}=\Theta_{1} \Theta_{2}^{-1} \Theta_{1} \Theta_{2}^{-1} \Theta_{1}$ obtained from the recursion operator $R=\Theta_{2} \Theta_{1}^{-1}$. Here $\Theta_{1}=u^{2} D u^{2}$ and $\Theta_{2}=u^{3} D^{3} u^{3}$ are the two Hamiltonian operators constituting the well known bi-Hamiltonian formulation for the Dym equation. These more elementary Hamiltonian operators will turn up in the reductions of the quadratic and the cubic bracket, too.

In a similar fashion, all Hamiltonian formulations associated with any Lax operator of the form (3.126) can be calculated for any $N$. In all cases one looses the Hamiltonian structure in the reductions (3.6.ii-iv). For the final reduction (3.6.v) Dirac reduction will lead to the Hamiltonian 
formulations for the equations associated with these Lax operators.

The quadratic tensor $\mathscr{P}_{2}$ in (2.8) does not define a Poisson bracket on $g$ for the present choice $k=2$. Nevertheless, it turns out that Hamiltonian formulations are hidden in this tensor for certain reductions. Considering the reduction properties we first look at the second representation for $\mathscr{P}_{2}$ in (3.105). Inserting $L$ of the form (3.126), the highest differential order of the operator $\mathscr{P}_{2}^{(k=2)}(L) \nabla H$ is easily seen to be at most $N$, matching the form of the highest differential orders of the ansatz (3.126). Concerning the lowest differential orders we insert $L$ of the form (3.126) into the first representation for $\mathscr{P}_{2}$ in (3.105). The second and third term will produce expressions of the form $-2\left(u_{1} \partial+u_{0}+\partial^{-1} u_{-1}+\partial^{-2} u_{-2}\right)\left(\partial^{-1} a_{-1}+\partial^{-2} a_{-2}\right)$ and $-2\left(\partial^{-1} a_{1}+\partial^{-2} a_{-2}\right)\left(\partial^{-1} u_{-1}+\partial^{-2} u_{-2}\right)$, where $a_{-1}, a_{-2}$ are the corresponding coefficients of the operator $[\nabla H, L]$. Such terms do not lie in the dual subalgebra $g_{<2}^{*}$, from which the Lax operators (3.126) were chosen. Hence, the quadratic bracket cannot be properly restricted to $g_{<2}^{*}$. However, choosing $L$ to be of the form

$$
L=u_{N} \partial^{N}+u_{N-1} \partial^{N-1}+. .+u_{2} \partial^{2}+\left(\lambda_{1} x+\lambda_{2}\right) \partial+\lambda_{3},
$$

the operator $\mathscr{P}_{2}^{(k=2)}(L) \nabla H$ will take values in $g_{<2}^{*}$. Still, expressions of first to minus second order, i.e. of the form (..) $\partial+(.)+.\partial^{-1}(.)+.\partial^{-2}(.$.$) , will be$ present. Hence, the quadratic bracket cannot be properly restricted to operators of the form (3.133), either. Nevertheless, these properties allow to apply Dirac reduction to operators (3.133) in a simple way. We demonstrate this considering the Lax operator $L=u^{2} \partial^{2}$ associated with the Dym equation (3.44), where all constant parameters are chosen to be zero. We think of $L$ as $L=u^{2} \partial^{2}+v \partial+w+\partial^{-1} z+\partial^{-2} r$, subject to the constraints $r=z=w=v=0$. Inserting $\nabla H=\frac{\delta H}{\delta r} \partial+\frac{\delta H}{\delta z}+\partial^{-1} \frac{\delta H}{\delta w}+\partial^{-2} \frac{\delta H}{\delta v}+\partial^{-3} \frac{1}{2 u} \frac{\delta H}{\delta u}$, the evolution equation $\frac{d}{d t} L=\mathscr{P}_{2}^{(k=2)}(L) \nabla H$ translates to 


$$
\begin{gathered}
d t\left(\begin{array}{c}
u \\
v \\
w \\
z \\
r
\end{array}\right)=2\left(\begin{array}{cccc}
0 & 0 & 2 u^{2} D u & 2 u^{2} D u D \\
0 & 4 u^{2} D u^{2} & 2 u^{2} D u^{2} D & 4 u^{2} D u D u D \\
2 u D u^{2} & -2 u D u^{2} D u^{2} & D u^{2} D u^{2}+u^{2} D^{2} u^{2} D & u^{2} D^{2} u^{2} D^{2} \\
-2 D u D u^{2} & 4 D u D u D u^{2} & -D^{2}+u^{2} D^{2} u^{2} & 0 \\
D^{2} u D u^{2} & -D^{2} u^{2} D^{2} u^{2} & 0 & 0 \\
u^{2} D u D^{2} \\
u^{2} D^{2} D^{2} \\
0 \\
0 \\
0
\end{array}\right)\left(\begin{array}{c}
\frac{\delta H}{\delta u} \\
\frac{\delta H}{\delta v} \\
\frac{\delta H}{\delta w} \\
\frac{\delta H}{\delta z} \\
\frac{\delta H}{\delta r}
\end{array}\right)
\end{gathered}
$$

The Dirac reduced equations reads

$$
\frac{d}{d t} u=8\left(u^{2} D u^{2}\right)\left(\frac{1}{u^{3}} D^{-3} \frac{1}{u^{3}}\right)\left(u^{2} D u^{2}\right) \frac{\delta H}{\delta u}
$$

Again, one encounters a formal Hamiltonian operator of the form $\Theta_{1} \Theta_{2}^{-1} \Theta_{1}$, where $\Theta_{1}$ and $\Theta_{2}$ are the basic bi-Hamiltonian operators of the Dym equation already encountered in the reduction of the linear bracket. Hence, despite the fact that the general quadratic bracket is not a Poisson structure, its Dirac reduction does lead to a Hamiltonian formulation of the Dym equation.

In a similar way the Dirac reduction of the quadratic bracket can be performed for any operator of the form (3.133). We expect that also for $N>2$ Hamiltonian formulations for the isospectral hierarchies for these operators can be obtained this way. However, just as in the case $k=1$, there is no structural justification for this construction as the general quadratic bracket does not define a Poisson structure.

The cubic tensor in (2.8) does define a Poisson bracket, so that the following considerations are well motivated. An analysis similar to the case of the quadratic bracket shows that only for Lax operators of the restricted 
class (3.133) the operator $\mathscr{P}_{3}^{(k=2)}(L) \nabla H$ will take values in $g_{<2}^{*}$. However, for the cubic bracket, this Hamiltonian vectorfield will actually take values in $g_{\geq 2} \subset g_{<2}^{*}$, if the constant parameters vanish. Hence, the cubic bracket can be properly restricted to the subalgebra $g_{>2}$. Considering the second representation for $\mathscr{P}_{3}$ in (3.105), one easily deduces that the highest differential order of $\mathscr{P}_{3}^{(k=2)}(L) \nabla H$ will be $\max (N, 2 N-3)$. Hence, there is only one simple Poisson subspace for the cubic bracket in the class (3.126), given by second order operators of the form $L=u^{2} \partial^{2}$. In this case, we insert $\nabla H=\partial^{-3} \frac{1}{2 u} \frac{\delta H}{\delta u}$ and $\frac{d}{d t} L=\mathscr{P}_{3}^{(k=2)}(L) \nabla H$ translates to the Hamiltonian formulation

$$
\frac{d}{d t} u=u^{2} D u^{2} \frac{\delta H}{\delta u}
$$

of the Dym hierarchy.

For $N>2$ Dirac reduction can be performed to obtain further Hamiltonian formulations of the isospectral hierarchies associated with Lax operators of the form (3.133).

Summarizing this section we point out that the abstract brackets (2.8) indeed encode the multi-Hamiltonian formulations of the nonlinear integrable systems defined by the Lax equations considered here. Depending on the reduction, either a simple restriction or Dirac reduction to the corresponding class of Lax operators will lead to the Hamiltonian formulations in a straightforward, although sometimes technical, way. As a surprising fact, also the quadratic bracket for $k=1$ and $k=2$ yields Hamiltonian structures. We believe that also the multi-Hamiltonian formulations of the Kupershmidt constrained systems of Section 3.3 can be obtained as Dirac reductions of the general brackets.

\section{$\S 4$. Sato Approach: Three Classes of Integrable Equations in $2+1$ Dimensions}

\section{§4.1. The KP Hierarchy and Nonstandard Sato Equations}

In this section we will copy the construction of the KP hierarchy within Sato's approach ([10-14]) for the three classes of equations (2.28). The idea essentially is that one considers Lax operators spanned by infinitely many 
fields. Hence, for fixed $k$ and varying $q$, each of the equations (2.28) represents a 1+1-dimensional integrable system with infinitely many fields. As all equations commute for different values of $q$, they may be considered simultaneously. Then one tries to extract a closed equation for a single field by eliminating the other fields using the evolution equations.

We consider operators of the form (3.3), where we assume that an infinite "tail" of integration symbols is present. Without loosing generality, we may assume $N=1$, as the $\operatorname{Lax}$ equation (2.28) for any $L=u_{N} \partial^{N}+$ lower for a given power $q$ leads to the same equation as the Lax operator $\tilde{L}=L^{\frac{1}{N}}=\left(u_{N}\right)^{\frac{1}{N}} \partial+$ lower with the power $\tilde{p}=N p$. Thus, the construction only depends on the choices of the non-dynamical leading order coefficients in $L$. For simplicity, we will again choose $c_{N}=1$ and $c_{N-1}=0$ as the leading orders in the cases $k=0$ and $k=1$. Further, we will fix $N=1$, and the powers $q$ in (2.28) will be restricted to the natural numbers. As we will consider the equations simultaneously, we introduce a different time variable $t_{n}$ for each choice of $q=n \in N$. Thus, we will consider the hierarchy of equations

$$
\frac{d}{d t_{n}} L=\left[P_{\geq k}\left(L^{n}\right), L\right], n=1,2,3, \cdots
$$

where we choose

$$
\underline{k=0:} \quad L_{K P}=\partial+\frac{1}{2} u \partial^{-1}+u_{2} \partial^{-2}+u_{3} \partial^{-3}+\cdots
$$

as in standard Sato theory, together with

$$
\begin{array}{ll}
\underline{k=1:} \quad L_{m K P}=\partial+v+v_{1} \partial^{-1}+v_{2} \partial^{-2}+v_{3} \partial^{-3}+\cdots \\
\underline{k=2:} \quad L_{D y m}=w \partial+w_{0}+w_{1} \partial^{-1}+w_{2} \partial^{-2}+w_{3} \partial^{-3}+\cdots .
\end{array}
$$

Here the "highest dynamical coefficients" $u, v$ and $w$ carry no index, since they will be taken as distinguished fields which satisfy identifiable integrable equations. These are the KP hierarchy for $k=0$, the modified KP hierarchy for $k=1$ and a 2+1-dimensional Dym hierarchy for $k=2$, as indicated by the choice of subscripts for the Lax operators. The remaining fields $u_{2}, u_{3}, \cdots, v_{1}, v_{2}, \cdots, w_{0}, w_{1}, \cdots$, may be regarded as auxiliary fields to be eliminated in the following construction. 


\section{The case $\boldsymbol{k}=\mathbf{0}$. The Kadomtsev-Petviashvili hierarchy}

Here, we review the construction of the KP hierarchy via Sato theory. Thus, with $L=L_{K P}=\partial+\frac{1}{2} u \partial^{-1}+u_{2} \partial^{-2}+u_{3} \partial^{-3}+\cdots$ one readily calculates the differential operators

$$
\begin{aligned}
& P_{\geq 0}(L)=\partial, \\
& P_{\geq 0}\left(L^{2}\right)=\partial^{2}+u, \\
& P_{\geq 0}\left(L^{3}\right)=\partial^{3}+\frac{3}{2} u \partial+\frac{3}{2} u_{x}+3 u_{2} .
\end{aligned}
$$

In general, it is clear that the operator $P_{\geq 0}\left(L^{n}\right)$ will be a differential operator of order $n$ involving the fields $u, u_{2}, u_{3}, \cdots, u_{n-1}$. Thus, the first of the evolution equations (4.1) become

$$
\begin{gathered}
L_{t_{1}}=\left[P_{\geq 0}(L), L\right] \leftrightarrow\left\{\begin{array}{l}
u_{t_{1}}=u_{x}, \\
u_{2 t_{1}}=u_{2 x}, \\
u_{3 t_{1}}=u_{3 x}, \\
\cdots,
\end{array}\right. \\
L_{t_{2}}=\left[P_{\geq 0}\left(L^{2}\right), L\right] \leftrightarrow\left\{\begin{array}{c}
u_{y}=u_{x x}+4 u_{2 x}, \\
u_{2 y}=u_{2 x x}+2 u_{3 x}+\frac{1}{2} u u_{x}, \\
u_{3 y}=u_{3 x x}+2 u_{4 x}-\frac{1}{2} u u_{x x}+2 u_{2} u_{x}, \\
\cdots,
\end{array}\right. \\
L_{t_{3}}=\left[P_{\geq 0}\left(L^{3}\right), L\right] \leftrightarrow\left\{\begin{array}{c}
u_{t}=u_{x x x}+3 u u_{x}+6 u_{2 x x}+6 u_{3 x}, \\
u_{2 t}=u_{2 x x x}+3\left(u u_{2}\right)_{x}+3 u_{3 x x}+3 u_{4 x}, \\
\cdots,
\end{array}\right.
\end{gathered}
$$

with the more convenient notation $y=t_{2}, t=t_{3}$. The first set of equations indicates, that we may identify $x=t_{1}$. These are the first equations of a countable hierarchy of commuting coupled equations involving all the fields $u, u_{2}, u_{3}, \cdots$. For each $n$, the corresponding evolution equation represents a $1+1$-dimensional system in the two independent variables $t_{n}$ and $x$ and infinitely many dependent fields. In Section 3 we have discussed reductions, in which only finitely many of these variables are present. Namely, we may assume $L$ to be of the form $L=\tilde{L}^{\frac{1}{N}}$, where $\tilde{L}=\partial^{N}+\tilde{u}_{N-2} \partial^{N-2}+. .+\tilde{u}_{0}$ is a purely differential operator. This is indeed an admissible reduction for the dynamics (4.1). Now all coefficients of $L$ can be expressed in terms of the $N-2$ 
dynamical fields $\tilde{u}_{N-2}, \cdots, \tilde{u}_{0}$ in $\widetilde{L}$, and (4.1) will be equivalent to the isospectral hierarchy for $\tilde{L}$ as constructed in Section 3 .

Another way of extracting equations out of (4.6), (4.7), , , which involve one field only makes use of the particular structure of the equations associated with $t_{2}=y$. In this connection, it is noted that by a simple integration with respect to $x$ the fields $u_{2}, u_{3}, \cdots$, can be recursively expressed in terms of the field $u$ and its derivatives with respect to $y$. Hence $u_{2}, u_{3}, \cdots$ may be considered as auxiliary fields to be eliminated via (4.6) from the higher equations in the above hierarchy. For example, eliminating $u_{2}$ and $u_{3}$ via (4.6), one can rewrite the first component of (4.7) in terms of $u$, its $x$ - and its $y$-derivatives. The equation thus encountered is the Kadomtsev-Petviashvili (KP) equation

$$
4 u_{t x}=\left(u_{x x x}+6 u u_{x}\right)_{x}+3 u_{y y}
$$

Elimination of the auxiliary fields from the other components of (4.7) just gives differential consequences of (4.8). One may also consider the higher equations for $u$ arising from $L_{t_{n}}=\left[P_{\geq 0}\left(L^{n}\right), L\right]$. These are of the form $u_{t_{n}}=E_{n}\left(u, u_{2}, u_{3}, \cdots, u_{n}\right)$ with some differential expression $E_{n}$ of the indicated arguments. After elimination of the auxiliary fields via (4.6) there remains an equation for $u$ only. Since it has to commute with (4.8), it represents the $n$th member of the $\mathbb{K P}$ hierarchy.

We remark that this elimination process can be simplified by directly considering the operators $P_{\geq 0}\left(L^{n}\right)$. Using the zero-curvature equation (2.17) the $n$th member of the $\mathbb{K P}$ hierarchy is encoded in the operator equation

$$
\frac{d}{d t_{n}}\left(P_{\geq 0}\left(L^{2}\right)\right)-\frac{d}{d y}\left(P_{\geq 0}\left(L^{n}\right)\right)+\left[P_{\geq 0}\left(L^{2}\right), P_{\geq 0}\left(L^{n}\right)\right]=0
$$

The lowest differential order defines the time evolution $u_{t_{n}}$ in terms of the auxiliary fields entering $P_{\geq 0}\left(L^{n}\right)$. The coefficients of the higher differential orders define the constraints, from which the auxiliary fields can be eliminated in terms of $u$ and its $y$-derivatives.

\section{The case $k^{\prime}=\mathbb{1}$. The modified Kadomtsev-Petviashvili hierarchy}

A similar analysis, now with $k=1$ and $L=L_{m K P}=\partial+v+v_{1} \partial^{-1}+v_{2} \partial^{-2}+\cdots$, leads to the modified $\mathrm{KP}$ hierarchy for the field $v$. In this case,

$$
P_{\geq 1}(L)=\partial,
$$




$$
\begin{aligned}
& P_{\geq 1}\left(L^{2}\right)=\partial^{2}+2 v \partial, \\
& P_{\geq 1}\left(L^{3}\right)=\partial^{3}+3 v \partial^{2}+3\left(v_{x}+v^{2}+v_{1}\right) \partial .
\end{aligned}
$$

In general, the operator $P_{\geq 1}\left(L^{n}\right)$ will be a differential operator of order $n$ which involves the fields $v, v_{1}, v_{2}, \cdots v_{n-2}$. The first of the evolution equations (4.1) are

$$
\begin{gathered}
L_{t_{1}}=\left[P_{\geq 1}(L), L\right] \leftrightarrow\left\{\begin{array}{c}
v_{t_{1}}=v_{x}, \\
v_{1 t_{1}}=v_{1 x}, \\
v_{2 t_{1}}=v_{2 x}, \\
\cdots,
\end{array}\right. \\
L_{t_{2}}=\left[P_{\geq 1}\left(L^{2}\right), L\right] \leftrightarrow\left\{\begin{array}{c}
v_{y}=v_{x x}+2 v v_{x}+2 v_{1 x}, \\
v_{1 y}=v_{1 x x}+2\left(v v_{1}\right)_{x}+2 v_{2 x}, \\
v_{2 y}=v_{2 x x}-2 v_{1} v_{x x}+2 v v_{2 x}+4 v_{x} v_{2}+2 v_{3 x}, \\
\cdots, \\
L_{t_{3}}=\left[P_{\geq 1}\left(L^{3}\right), L\right]
\end{array}\right. \\
\hline\left\{\begin{array}{c}
v_{t}=v_{x x x}+3 v_{1 x x}+3 v_{2 x}+3\left(v v_{x}\right)_{x}+3 v^{2} v_{x}+6\left(v v_{1}\right)_{x}, \\
\cdots,
\end{array}\right.
\end{gathered}
$$

where again we have set $y=t_{2}, t=t_{3}$. Now the auxiliary fields $v_{1}, v_{2}, \cdots$, can be eliminated by expressing them in terms of the field $v$ and its $y$-derivatives. For example, eliminating $v_{1}$ and $v_{2}$ via (4.12), one can rewrite the first component of (4.13) in terms of $v$. The equation so obtained is the modified Kadomtsev-Petviashvili (mKP) equation

$$
4 v_{t x}=\left(v_{x x x}-6 v^{2} v_{x}\right)_{x}+3 v_{y y}+6 v_{x} v_{y}+6 v_{x x} D^{-1} v_{y}
$$

Here $D^{-1}=\int^{x} d \xi$ is the integration operator arising from the elimination of the auxiliary fields. On consideration of the higher equations for $v$ arising from $L_{t_{n}}=\left[P_{\geq 1}\left(L^{n}\right), L\right]$ with $n=4,5, \cdots$, one finds $v_{t_{n}}=E_{n}\left(v, v_{1}, v_{2}, \cdots, v_{n-1}\right)$ where the expression $E_{n}$ involves derivatives of the indicated arguments. After elimination of the auxiliary fields via (4.12) an equation for $v$ only remains. This represents the $n$th member of the modified KP hierarchy. Again, the necessary calculations are simplified by directly considering the corresponding zero-curvature equations (4.9) (with $P_{\geq 0}$ replaced by $P_{\geq 1}$ ).

\section{The case $k=2$. The Dym hierarchy in $2+1$ dimensions}

A similar analysis, but now with $k=2$ and $L=L_{D y m}=w \partial+w_{0}+w_{1} \partial^{-1}+$ 
$w_{2} \partial^{-2}+\cdots$, leads to a hierarchy of $2+1$-dimensional Dym equations for the field $w$. Here

$$
\begin{aligned}
& P_{\geq 2}(L)=0, \\
& P_{\geq 2}\left(L^{2}\right)=w^{2} \partial^{2}, \\
& P_{\geq 2}\left(L^{3}\right)=w^{3} \partial^{3}+3 w^{2}\left(w_{x}+w_{0}\right) \partial^{2} .
\end{aligned}
$$

In general, the operator $P_{\geq 2}\left(L^{n}\right)$ will be a differential operator of order $n$ involving the fields $w, w_{0}, w_{1}, \cdots w_{n-3}$. The first of the evolution equations (4.1) are

$$
\begin{aligned}
& L_{t_{1}}=\left[P_{\geq 2}(L), L\right] \leftrightarrow\left\{\begin{array}{c}
w_{t_{1}}=0, \\
w_{0 t_{1}}=0, \\
\cdots,
\end{array}\right. \\
& L_{t_{2}}=\left[P_{\geq 2}\left(L^{2}\right), L\right] \leftrightarrow\left\{\begin{array}{c}
w_{y}=w^{2} w_{x x}+2 w^{2} w_{0 x}, \\
w_{0 y}=w^{2} w_{0 x x}+2 w\left(w w_{1}\right)_{x}, \\
w_{1 y}=w^{2} w_{1 x x}+2\left(w^{2} w_{2}\right)_{x}-2 w_{1}\left(w w_{x}\right)_{x}, \\
\cdots,
\end{array}\right. \\
& L_{t_{3}}=\left[P_{\geq 2}\left(L^{3}\right), L\right] \leftrightarrow\left\{\begin{array}{c}
w_{t}=w^{2}\left(\frac{1}{2}\left(w^{2}\right)_{x x}+3\left(w w_{0}\right)_{x}+3 w_{0}^{2}+3 w w_{1}\right)_{x}, \\
\cdots,
\end{array}\right.
\end{aligned}
$$

where again $y=t_{2}, t=t_{3}$. Now the auxiliary fields $w_{0}, w_{1}, \cdots$, can be eliminated by expressing them in terms of the field $w$ and its $y$-derivatives. For instance, eliminating $w_{0}$ and $w_{1}$ via (4.17), one can rewrite the first component of (4.18) in terms of $w$. The equation so derived is a $2+1$-dimensional version of the Dym equation first constructed in Ref. [23]:

$$
4 w_{t}=w^{3} w_{x x x}-3 \frac{1}{w}\left(w^{2} D^{-1}\left(\frac{1}{w}\right)_{y}\right)_{y}
$$

Again, the integration $D^{-1}=\int^{x} d \xi$ arises from the elimination of the auxiliary fields.

As before, considering the higher equations for $w$ arising from $L_{t_{n}}=\left[P_{\geq 2}\left(L^{n}\right), L\right]$ with $n=4,5, \cdots$, one finds $w_{t_{n}}=E_{n}\left(w, w_{0}, w_{1}, \cdots, w_{n-2}\right)$ with expressions $E_{n}$ involving the derivatives of the indicated arguments. After 
elimination of the auxiliary fields by (4.17) there remains an equation for $w$ only. This represents the $n$-th member of the $2+1$-dimensional Dym hierarchy. Again, the necessary calculations are simplified by directly considering the corresponding zero-curvature equations (4.9) (with $P_{\geq 0}$ replaced by $P_{\geq 2}$ ).

\section{§4.2. Kupershmidt Reductions}

As the 2+1-dimensional hierarchies considered here really are just reinterpretations of the 1+1-dimensional hierarchies (4.1) for infinitely many fields, we can apply the results of Section 3.3 to the present considerations, too. In particular, we can impose the constraints (3.63) with $N=1$, and each second equation in (4.1) with odd $n$ will survive this reduction. Hence we consider

$$
\frac{d}{d t_{n}} L=\left[P_{\geq k}\left(L^{n}\right), L\right], \quad n=1,3,5, \cdots, \quad L+\partial^{-k} L^{\dagger} \partial^{k}=0
$$

The constraint implies relations between the auxiliary fields of the operators (4.2) and (4.3). In particular, we parametrize the Lax operators in the following way:

$$
\begin{aligned}
\underline{k=0:} \quad L & =\partial+u \partial^{-1}+\partial^{-1} u+u_{3} \partial^{-3}+\partial^{-3} u_{3}+u_{5} \partial^{-5}+\partial^{-5} u_{5}+\cdots \\
& =\partial+2 u \partial^{-1}-u_{x} \partial^{-2}+\left(2 u_{3}+u_{x x}\right) \partial^{-3}-\left(3 u_{3 x}+u_{x x x}\right) \partial^{-4}+\cdots, \\
\underline{k=1:} \quad L & =\partial^{-1}\left(\partial^{2}+v+v_{3} \partial^{-2}+\partial^{-2} v_{3}+v_{5} \partial^{-4}+\partial^{-4} v_{5}+\cdots\right) \\
& =\partial+v \partial^{-1}-v_{x} \partial^{-2}+\left(2 v_{3}+v_{x x}\right) \partial^{-3}-\left(4 v_{3 x}+v_{x x x}\right) \partial^{-4}+\cdots, \\
\underline{k=2: \quad L} & =\partial^{-2}\left(w \partial^{3}+\partial^{3} w+w_{1} \partial+\partial w_{1}+w_{3} \partial^{-1}+\partial^{-1} w_{3}+\cdots\right) \\
& =2 w \partial-w_{x}+\left(2 w_{1}+3 w_{x x}\right) \partial^{-1}-\left(3 w_{1 x}+4 w_{x x x}\right) \partial^{-2}+\cdots,
\end{aligned}
$$

so that the constraints are satisfied automatically. In all cases the distinguished fields are those connected with the highest dynamical differential order of $L$. All other fields are auxiliary fields to be eliminated in the following construction. The relevant differential operators $P_{\geq k}\left(L^{3}\right)$ and $P_{\geq k}\left(L^{5}\right)$ are calculated as

$$
\begin{array}{ll}
k=0: & P_{\geq 0}\left(L^{3}\right)=\partial^{3}+3 u \partial+3 \partial u, \\
& P_{\geq 0}\left(L^{5}\right)=\partial^{5}+5 u \partial^{3}+5 \partial^{3} u+\left(20 u^{2}+5 u_{3}\right) \partial+\partial\left(20 u^{2}+5 u_{3}\right),
\end{array}
$$




$$
\begin{aligned}
& \text { k=1: } \quad P_{\geq 1}\left(L^{3}\right)=\partial^{3}+3 v \partial, \\
& P_{\geq 1}\left(L^{5}\right)=\left(\partial^{4}+5 v \partial^{2}+5 \partial^{2} v-5 \partial v \partial+10\left(v^{2}+v_{3}\right)\right) \partial, \\
& \underline{k=2:} \quad P_{\geq 2}\left(L^{3}\right)=4\left(w^{3} \partial+\partial w^{3}\right) \partial^{2} \text {, } \\
& P_{\geq 2}\left(L^{5}\right)=\left(6\left(w^{5} \partial^{3}+\partial^{3} w^{5}\right)+50\left(w^{4} \partial^{3} w+w \partial^{3} w^{4}\right)-40\left(\partial^{2} w^{2} \partial w^{3}\right.\right. \\
& \left.\left.+w^{3} \partial w^{2} \partial^{2}\right)+80\left(\partial w^{4} w_{1}+w^{4} w_{1} \partial\right)\right) \partial^{2} .
\end{aligned}
$$

Now, for $k=0$ the Lax equation (4.20) associated with $y=t_{3}$ imply evolutions of the form $u_{y}=E_{1}\left(u, u_{3}\right), u_{3 y}=E_{3}\left(u, u_{3}, u_{5}\right), \cdots$, with certain differential expressions $E_{i}$ of the indicated arguments. We can use these relations to express all the auxiliary fields $u_{3}, u_{5}, \cdots$, in terms of the field $u$ and its $y$-derivatives. Eliminating the auxiliary fields from the equation associated with $t=t_{5}$ one obtains an equations for the distinguished field $u$, only. In exactly the same fashion 2+1-dimensional equations for the distinguished fields $v$ and $w$ can be extracted for the cases $k=1$ and $k=2$, too. Alternatively, one may directly use the zero-curvature condition

$$
\frac{d}{d t}\left(P_{\geq k}\left(L^{3}\right)\right)-\frac{d}{d y}\left(P_{\geq k}\left(L^{5}\right)\right)+\left[P_{\geq k}\left(L^{3}\right), P_{\geq k}\left(L^{5}\right)\right]=0
$$

to derive the relations between the auxiliary fields and the prime fields as well as the $t_{5}$-evolution for the prime fields. The resulting equations are

$$
\begin{aligned}
& \underline{k=0:} \quad 9 u_{t}+u_{x x x x x}+ 30 u u_{x x x}+75 u_{x} u_{x x}+180 u^{2} u_{x}-5 u_{y x x}-5 D^{-1} u_{y y} \\
&-30\left(u D^{-1} u_{y}\right)_{x} \\
& k=1: \quad 9 v_{t}+v_{x x x x x}+ 15 v v_{x x x}+15 v_{x} v_{x x}+45 v^{2} v_{x}-5 v_{y x x}-5 D^{-1} v_{y y} \\
&-15\left(v D^{-1} v_{y}\right)_{x} \\
& k \\
& k=2: \quad 18 w_{t}+64 w^{5} w_{x x x x x}+320 w^{4} w_{x} w_{x x x x}+160 w^{4} w_{x x} w_{x x x}+240 w^{3} w_{x}^{2} w_{x x x} \\
&--80 w^{\frac{5}{2}}\left(w^{\frac{1}{2}}\right)_{y x x}-\frac{5}{w^{2}}\left(w^{3} D^{-1} \frac{w_{y}}{w^{2}}\right)_{y}
\end{aligned}
$$

These equations were found in Ref. [23]. If we impose the additional constraint $L^{3}=P_{\geq k}\left(L^{3}\right)$, then these equations reduce to their $1+1$-dimensional counterparts associated with the third order Lax operators $\widetilde{L}=L^{3}$. In particular, in this reduction the $y$-evolution will become trivial. Hence, omitting the $y$-derivatives in (4.24) and using a suitable rescaling of the fields, these equations reduce to the Kaup-Kupershmidt equation (3.65), the Sawada-Kotera equation (3.77) and the equation (3.96), $\lambda_{1}=0$, respectively. The hierarchies of the equations (4.24) are obtained from (4.23), when 
replacing $t=t_{5} \rightarrow t_{n}, L^{5} \rightarrow L^{n}, n=7,9, \ldots$.

\section{§5. Gauge Transformations and Reciprocal Links: Darboux Theorems}

In Section 4 we have identified the three integrable hierarchies $\frac{d}{d t_{q}} L=\left[P_{\geq k}\left(L^{q}\right), L\right], k=0,1,2$ as the KP hierarchy, the modified KP hierarchy and the 2+1-dimensional Dym hierarchy, respectively. The constrained equations of Section 4.2 and all the 1+1-dimensional systems of Section 3 may be regarded as reductions of these general hierarchies. It is well known that these three hierarchies are connected via Miura type transformations ([34]) and reciprocal links ([35]), respectively. We will now show, that these relations are actually built into the construction of these hierarchies using the three different projections $P_{\geq k}$. The relations between the classes $k=0$ and $k=1$ had already been observed in Ref. [21], a general analysis was given in Ref. [24]. Here we briefly review the results of Ref. [24] and then discuss some of the reduction aspects to the $1+1$-dimensional hierarchies of Section 3 .

The Lax equations $\frac{d}{d t_{q}} L=\left[P_{\geq k}\left(L^{q}\right), L\right]$ guarantee the compatibility of the linear problems

$$
L \Phi=\lambda \Phi, \Phi_{t_{q}}=P_{\geq k}\left(L^{q}\right) \Phi,
$$

which may be considered simultaneously for arbitrary $q$ because of the zero-curvature equations (2.17). In the case of 2+1-dimensional equations the evolution equations for $\Phi$ are in fact the linear problems from which an Inverse Scattering Method for the corresponding nonlinear evolution equation may be derived. For instance, for the KP equation (4.8) the linear problems are given by $\Phi_{y}=P_{\geq 0}\left(L^{2}\right) \Phi$ and $\Phi_{t}=P_{\geq 0}\left(L^{3}\right) \Phi$, which involve the potential $u$ satisfying the KP equation. The auxiliary field $u_{2}$ in $P_{\geq 0}\left(L^{3}\right)$ has to be thought of as being defined in terms of $u$ and its $y$-derivative via (4.6). For the reduction to the 1+1-dimensional equations one imposes the eigenvalue problem $L \Phi=\lambda \Phi$ as an additional constraint, which is compatible with the time evolutions of $\Phi$ given by (5.1).

It turns out that Miura transformations as well as reciprocal links are most conveniently generated from Darboux theorems involving eigenfunctions of the associated Lax operators. The basic property of the eigenfunctions 
$\Phi$ are the time-evolutions given by (5.1), whereas the eigenvalue problem $L \Phi=\lambda \Phi$ may be considered as an additional constraint. We fix this notion of "eigenfunctions" with:

Definition 1. For given $k=0,1$, or 2 , a function $\Phi=\Phi\left(x, t_{q}\right)$ satisfying the linear equations

$$
\Phi_{t_{q}}=P_{\geq k}\left(L^{q}\right) \Phi
$$

shall be called an eigenfunction for the hierarchy of Lax equations $\frac{d}{d t_{q}} L=$ $\left[P_{\geq k}\left(L^{q}\right), L\right]$

We shall use the term "reciprocal link" in a general way as referring to a transformation involving a change of the independent variables. These transformations will generalize the links found in Ref. [36], which are truly reciprocal in the sense that they map Dym solutions $w$ to new solutions via $w \rightarrow w^{-1}$ (and a suitable change of the independent coordinates). The precise meaning of such a "reciprocal" link between Lax operators is given by

Definition 2. With a given function $\Phi\left(x, t_{q}\right)$ we introduce new independent variables $x^{\prime}=\Phi\left(x, t_{q}\right), t_{q}^{\prime}=t_{q}$. To each function $a\left(x, t_{q}\right)$ we associate the "linked" function $a^{\prime}\left(x^{\prime}, t_{q}^{\prime}\right)$ defined by $a^{\prime}\left(x^{\prime}, t_{q}^{\prime}\right)=a\left(x, t_{q}\right)$. By $\partial=\partial_{x}, \partial^{\prime}=\partial_{x^{\prime}}$ we denote the differential operators associated with the different variables. Observing $\partial=\Phi_{x} \partial^{\prime}$ we associate the operator $A^{\prime}=\sum_{i} a_{i}^{\prime}\left(x^{\prime}, t_{q}^{\prime}\right)\left(\Phi_{x} \partial^{\prime}\right)^{i}$ to a given pseudodifferential operator $A=\sum_{i} a_{i}\left(x, t_{q}\right) \partial^{i}$. We call the pair $A^{\prime}\left(x^{\prime}, t_{q}^{\prime}\right)=A\left(x, t_{q}\right)$ "linked" by the transformation $x^{\prime}=\Phi\left(x, t_{q}\right)$. We denote by $P_{\geq k}^{\prime}$ the "linked" projection operator $P_{\geq k}^{\prime}\left(\sum_{i} b_{i}^{\prime}\left(x^{\prime}, t_{q}^{\prime}\right) \partial^{\prime i}\right)=\sum_{i \geq k} b_{i}^{\prime}\left(x^{\prime}, t_{q}^{\prime}\right) \partial^{\prime i}$, related to the powers of the new symbol $\partial^{\prime}$.

With these notions it was shown in Ref. [24] that the three integrable hierarchies are related in the following way:

\section{Theorem $\mathbb{1}$.}

i) (gauge $k=0 \rightarrow k=1$ )

Let $L \in g$ satisfy the hierarchy of Lax equations $\frac{d}{d t_{q}} L=\left[P_{\geq 0}\left(L^{q}\right), L\right]$. Let $\Phi \neq 0$ be an eigenfunction of this hierarchy. Then $\tilde{L}=\Phi^{-1} L \Phi$ satisfies the 
hierarchy $\frac{d}{d t_{q}} \tilde{L}=\left[P_{\geq 1}\left(\tilde{L}^{q}\right), \tilde{L}\right]$. If $\Psi$ is a further eigenfunction for $L$, then $\widetilde{\Psi}=\Phi^{-1} \Psi$ is an eigenfunction for $\tilde{L}$, that is, $\tilde{\Psi}_{t_{q}}=P_{\geq 1}\left(\tilde{L}^{q}\right) \widetilde{\Psi}$

ii) (link $k=1 \rightarrow k=2$ )

Let $L=L\left(x, t_{q}\right)$ satisfy $\frac{d}{d t_{q}} L=\left[P_{\geq 1}\left(L^{q}\right), L\right]$. Let $\Phi\left(x, t_{q}\right), \Phi_{x} \neq 0$, be an eigenfunction of this hierarchy. Then $L^{\prime}\left(x^{\prime}, t_{q}^{\prime}\right)=L\left(x, t_{q}\right)$, linked by the transformation $x^{\prime}=\Phi\left(x, t_{q}\right), t_{q}^{\prime}=t_{q}$, satisfies the hierarchy $\frac{d}{d t_{q}^{\prime}} L^{\prime}=\left[P_{\geq 2}^{\prime}\left(L^{\prime q}\right), L^{\prime}\right]$. If $\Psi\left(x, t_{q}\right)$ is a further eigenfunction for $L$, then $\Psi^{\prime}\left(x^{\prime}, t_{q}^{\prime}\right)=\Psi\left(x, t_{q}\right)$ is an eigenfunction for $L^{\prime}$, that is, $\Psi_{t_{q}^{\prime}}^{\prime}=P_{\geq 2}^{\prime}\left(L^{\prime q}\right) \Psi^{\prime}$.

It was shown in Ref. [24] that these transformations on the Lax operators encode the well known Miura transformation between the KP equation (4.8) and the modified KP equation (4.14) as well as the reciprocal link connecting solutions of the modified KP equation and the 2+1-dimensional Dym equation (4.19). Here we are interested in the reductions of the general hierarchies to Lax operators associated with 1+1-dimensional integrable equations. In particular, we consider Lax operators of the form

$$
\begin{array}{ll}
\underline{k=0:} & L=\partial^{N}+u_{N-2} \partial^{N-2}+. .+u_{1} \partial+u_{0}, \\
\underline{k=1:} & \tilde{L}=\partial^{N}+v_{N-1} \partial^{N-1}+. .+v_{1} \partial+\lambda, \\
\underline{k=2:} & L^{\prime}=w_{N} \partial^{\prime N}+w_{N-1} \partial^{\prime N-1}+. .+w_{2} \partial^{\prime 2}+\lambda_{1} x \partial^{\prime}+\lambda_{2},
\end{array}
$$

each of which is spanned by $N-2$ dynamical fields $\left(u_{N-2}, . ., u_{0}\right),\left(v_{N-1}, . ., v_{1}\right)$ and $\left(w_{N}, \ldots, w_{2}\right)$, respectively. Here $\lambda, \lambda_{1,2}$ are to be constant parameters.

First considering the transformations from $k=0$ to $k=1$, one performs the gauge transformation $L \rightarrow \tilde{L}=\Phi^{-1} L \Phi$ with an eigenfunction $\Phi$ satisfying $L \Phi=\lambda \Phi$. The transformed operator will be of the form

$$
\tilde{L}=\partial^{N}+N \frac{\Phi_{x}}{\Phi} \partial^{N-1}+\left(u_{N-2}+\frac{N(N-1)}{2} \frac{\Phi_{x x}}{\Phi}\right) \partial^{N-2}+. .+(. .) \partial+\lambda
$$

The zero order coefficient $v_{0}$ of $\tilde{L}=\sum_{i} v_{i} \partial^{i}$ is of the form $v_{0}=\Phi^{-1}(L \Phi)=\lambda$, because we have assumed $\Phi$ to be a proper eigenfunction of $L$. Identifying $v_{N-1}=N \Phi^{-1} \Phi_{x}, v_{N-2}=u_{N-2}+\frac{1}{2} N(N-1) \Phi^{-1} \Phi_{x x}, . ., v_{0}=\lambda$, the operator $\tilde{L}$ is 
of the form $(5.3, k=1)$. If $L$ satisfies the $k=0$ hierarchy, then $\tilde{L}$ satisfies the $k=1$ hierarchy according to Theorem 1.i) and we have a relation between the isospectral hierarchies associated with $L$ and $\tilde{L}$ in terms of an eigenfunction $\Phi$ of $L$. Using the additional constraint $L \Phi=\lambda \Phi$ on the eigenfunction $\Phi$, one can eliminate $\Phi$ from the transformation and obtains a Miura transformation between the fields $\left(u_{N-2}, . ., u_{0}\right)$ and $\left(v_{N-1}, \ldots, v_{1}\right)$.

We demonstrate this procedure for the simplest case $N=2$, where one starts with the Schrödinger operator $L=\partial^{2}+u$ giving rise to the $\mathrm{KdV}$ hierarchy. The gauge transformed operator is calculated as $\tilde{L}=\Phi^{-1} L \Phi=$ $\partial+2 \Phi^{-1} \Phi_{x} \partial+\lambda$, giving rise to the hierarchy of the modified $\mathrm{KdV}$ equation (2.37) for the field $v=\Phi^{-1} \Phi_{x}$. Hence, the spatial part of the celebrated Miura transformation is encoded in the transformation

$$
v=\frac{\Phi_{x}}{\Phi}, \quad \Phi_{x x}+u \Phi=\lambda \Phi
$$

Indeed, eliminating $\Phi$ from this transformation one finds $u=\lambda-v_{x}+v^{2}$, i.e. the Miura relation (2.39) mapping solutions $v$ of the modified $\mathrm{KdV}$ (2.37) to solutions $u$ of the $\mathrm{KdV}$ (2.34).

Similarly, considering the transformations $k=1 \rightarrow k=2$, one starts with an operator $\widetilde{L}$ of the form $(5.3, k=1)$. Using an eigenfunction $\Psi$ satisfying $\tilde{L} \Psi=\mu \Psi$, one performs the transformation $x^{\prime}=\Psi$. Using $\partial=\Psi_{x} \partial^{\prime}$, the linked operator $L^{\prime}=\widetilde{L}$ is calculated to be of the form

$$
\begin{aligned}
L^{\prime}= & \Psi_{x}^{N} \partial^{\prime N}+\left(v_{N-1} \Psi_{x}^{N-1}+\frac{N(N-1)}{2} \Psi_{x}^{N-2} \Psi_{x x}\right) \partial^{\prime N-2}+. .+(. .) \partial^{\prime 2} \\
& +(\mu-\lambda) x^{\prime} \partial^{\prime}+\lambda .
\end{aligned}
$$

It is readily seen that the zero order term $w_{0}$ of $L^{\prime}=\sum_{i} w_{i} \partial^{\prime i}$ coincides with the zero order term of $\tilde{L}$, that is, $w_{0}=\lambda$. The first order term $w_{1}$ of $L^{\prime}$ is calculated from $w_{1}=\left(L^{\prime}-\lambda\right) x^{\prime}=(\tilde{L}-\lambda) \Psi=(\mu-\lambda) \Psi=(\mu-\lambda) x^{\prime}$. Identifying $w_{N}=\Phi_{x}^{N}, \quad w_{N-1}=v_{N-1} \Psi_{x}^{N-1}+\frac{1}{2} N(N-1) \Psi_{x}^{N-2} \Psi_{x x}, . ., w_{1}=(\mu-\lambda) x^{\prime}, w_{0}=\lambda$, the operator $L^{\prime}$ is of the form $(5.3, k=2)$. If $\tilde{L}$ satisfies the $k=1$ hierarchy, then according to Theorem 1.ii) the operator $L^{\prime}$ : satisfies the $k=2$ hierarchy in the coordinate $x^{\prime}$. Thus we have a reciprocal transformation from the fields $\left(v_{N-1}, \ldots, v_{1}\right)$ to the fields $\left(w_{N}, . ., w_{2}\right)$ satisfying the isospectral hierarchies associated with the operators $\tilde{L}$ and $L^{\prime}$. This transformation involves the 
eigenfunction $\Psi$ of $\widetilde{L}$, which can be eliminated using the additional constraint $\tilde{L} \Psi=\mu \Psi$.

We demonstrate this for the simplest case $N=2$. Starting with the operator $\widetilde{L}=\partial^{2}+2 v \partial+\lambda$ giving rise to modified $\mathrm{KdV}$ hierarchy one introduces $x^{\prime}=\Psi$ and calculates the linked operator $L^{\prime}=\Psi_{x}^{2} \partial^{\prime 2}+(\mu-\lambda) x^{\prime} \partial^{\prime}+\lambda$. Hence, the field $w=\Psi_{x}$ satisfies the hierarchy of the Dym equation (2.38) in the coordinate $x^{\prime}$, if $v$ satisfies the modified $\mathrm{KdV}$ hierarchy and $\Psi$ satisfies the corresponding linear equations. The transformation is encoded in the linking relations

$$
x^{\prime}=\Psi, \quad w=\Psi_{x}, \quad \Psi_{x x}+2 v \Psi_{x}=(\mu-\lambda) \Psi
$$

from which the eigenfunction can be eliminated. Thus, the reciprocal links between the hierarchies of the modified $\mathrm{KdV}$ and the Dym equation are found to be characterized by

$$
\frac{\partial x}{\partial x^{\prime}}=\frac{1}{w}, \quad t_{q}^{\prime}=t_{q}, \quad 2 v=(\mu-\lambda) \frac{x^{\prime}}{w}-w_{x^{\prime}}
$$

mapping solutions of the Dym hierarchy to solutions of the modified $\mathrm{KdV}$ hierarchy. For each member of the hierarchy the transformation has to be supplemented by the condition $\frac{\partial x}{\partial t_{q}^{\prime}}=-\frac{1}{w} \frac{d x^{\prime}}{d t_{q}}$, where $\frac{d x^{\prime}}{d t_{q}}=\Psi_{t_{q}}=P_{\geq 1}\left(L^{q}\right) \Psi$ can be expressed in terms of the Dym solution $w$ via (5.8). For the Dym equation and the modified $\mathrm{KdV}$ equation

$$
4 w_{t^{\prime}}=w^{3} w_{x^{\prime} x^{\prime} x^{\prime}}+3(\mu-\lambda)^{2} x^{\prime}\left(1-\frac{x^{\prime} w_{x^{\prime}}}{w}\right), 4 v_{t}=v_{x x x}-6 v^{2} v_{x}+6 \lambda v_{x}
$$

themselves one has $q=\frac{3}{2}$ and

$$
\begin{aligned}
\frac{\partial x^{\prime}}{\partial t} & =\Psi_{t}=\Psi_{x x x}+3 v \Psi_{x x}+\frac{3}{2}\left(v_{x}+v^{2}+\lambda\right) \Psi_{x} \\
& =\frac{1}{8}\left(w^{2} w_{x^{\prime} x^{\prime}}-2 w w_{x^{\prime}}^{2}+6(\mu+\lambda) w+3(\mu-\lambda)^{2} \frac{x^{\prime 2}}{w}\right)
\end{aligned}
$$

provides the time-dependence of the transformation between these two 
equations.

We remark that a reciprocal link between the modified $K d V$ and the Dym equation is well known from Painleve expansions of the modified $\mathrm{KdV}$ ([31]). There the transformation of the independent variable is given by $x^{\prime}=\Phi$, where $\Phi$ is the singularity field of the expansion satisfying the "singularity manifold equation" (2.40). It might be surprising that here the transformation $x^{\prime}=\Psi$ is given in terms of an eigenfunction $\Psi$ of the underlying scattering problem for the modified $\mathbb{K d V}$. The explanation is the following: One considers $\widetilde{L} \Psi=\mu \Psi$ and $\Psi_{t}=P_{\geq 1}\left(\widetilde{L}^{\frac{3}{2}}\right) \Psi$, i.e.

$$
\Psi_{x x}+2 v \Psi_{x}+\lambda \Psi=\mu \Psi, \quad \Psi_{t}=\Psi_{x x x}+3 v \Psi_{x x}+\frac{3}{2}\left(v_{x}+v^{2}+\lambda\right) \Psi
$$

and eliminates the field $v$ using the eigenvalue problem. Thus, one finds the nonlinear evolution equation

$$
4 \frac{\Psi_{t}}{\Psi_{x}}=\{\Psi ; x\}+3(\mu+\lambda)+\frac{3}{2}(\mu-\lambda)^{2}\left(\frac{\Psi}{\Psi_{x}}\right)^{2}
$$

for the eigenfunction. For $\mu=\lambda$ this coincides with the singularity equation (2.40), so that the eigenfunction $\Psi$ can be thought of as the singularity field in this case. We remark that the present approach using eigenfunctions of the Lax operators yields a more general result on such reciprocal links, as the considerations above are not restricted to the modified $\mathrm{KdV} / \mathrm{Dym}$ case associated with second order Lax operators. We will find similar results for all hierarchies associated with scattering operators of the type $(5.3, k=1,2)$.

It was observed in Ref. [24] that also different types of gauge transformations and reciprocal links may be considered for the Lax operators. In particular, one has

Theorem 2.

i) (gauge $k=0 \rightarrow k=0$ )

Let $L \in g$ satisfy the hierarchy of Lax equations $\frac{d}{d t_{q}} L=\left[P_{\geq 0}\left(L^{q}\right), L\right]$. Let $\Phi \neq 0$ be an eigenfunction of this hierarchy. Then $\hat{L}=\Phi \partial \Phi^{-1} L \Phi \partial^{-1} \Phi^{-1}$ again satisfies the hierarchy $\frac{d}{d t_{q}} \hat{L}=\left[P_{\geq 0}\left(\hat{L}^{q}\right), \hat{L}\right] . \quad$ If $\Psi$ is a further eigenfunction 
of $\mathrm{L}$, then $\hat{\Psi}=\Phi\left(\Phi^{-1} \Psi\right)_{x}$ is an eigenfunction for $\hat{L}$, that is, $\hat{\Psi}_{t_{q}}=P_{\geq 0}\left(\hat{L}^{q}\right) \hat{\Psi}$.

ii) (gauge $k=1 \rightarrow k=1$ )

Let $\tilde{L} \in g$ satisfy the hierarchy of Lax equations $\frac{d}{d t} \tilde{L}=\left[P_{\geq 1}\left(\tilde{L}^{q}\right), \widetilde{L}\right]$. Let $\widetilde{\Phi} \neq 0$ be an eigenfunction of this hierarchy. Then $\stackrel{\tilde{\tilde{L}}}{=} \widetilde{\Phi}_{x}^{-1} \tilde{\Phi}^{2} \partial \widetilde{\Phi}^{-1} \tilde{L} \widetilde{\Phi} \partial^{-1}$ $\widetilde{\Phi}_{x} \tilde{\Phi}^{-2}$ satisfies $\frac{d}{d t_{q}} \hat{\tilde{L}}=\left[P_{\geq 1}\left(\hat{\tilde{L}}{ }^{q}\right), \hat{\tilde{L}}\right]$. If $\tilde{\Psi}$ is a further eigenfunction for $\tilde{L}$, then $\hat{\tilde{\Psi}}=\widetilde{\Phi}_{x}^{-1}\left(\tilde{\Phi}_{x} \tilde{\Psi}-\widetilde{\Phi} \tilde{\Psi}_{x}\right)$ is an eigenfunction for $\tilde{\tilde{L}}$, that is, $\hat{\Psi}_{t_{q}}=P_{\geq 1}$ $(\hat{\tilde{L}})^{q} \hat{\tilde{\Psi}}$.

iii) (link $k=2 \rightarrow k=2$ )

Let $L^{\prime}=L^{\prime}\left(x^{\prime}, t_{q}^{\prime}\right)$ satisfy $\frac{d}{d t_{q}^{\prime}} L^{\prime}=\left[P_{\geq 1}^{\prime}\left(L^{\prime q}\right), L^{\prime}\right]$. Let $\Phi^{\prime}=\Phi^{\prime}\left(x^{\prime}, t_{q}^{\prime}\right)$ be an eigenfunction of this hierarchy. Then $\hat{L}^{\prime}\left(\hat{x}^{\prime}, t_{q}^{\prime}\right)=\Phi_{x^{\prime}}^{\prime-1} \Phi^{\prime 2} \partial^{\prime} \Phi^{\prime-1} L^{\prime} \Phi^{\prime} \partial^{\prime-1} \Phi_{x^{\prime}}^{\prime} \Phi^{\prime-2}$, linked via the transformation $\hat{x}^{\prime}=x^{\prime}-\Phi_{x^{\prime}}^{\prime-1} \Phi^{\prime}, \hat{t}_{q}^{\prime}=t_{q}^{\prime}$, satisfies the hierarchy $\frac{d}{d \hat{t}_{q}^{\prime}} \hat{L}^{\prime}=\left[P_{\geq 2}^{\prime}\left(\hat{L}^{\prime q}\right), \hat{L}^{\prime}\right] . \quad$ If $\Psi^{\prime}\left(x^{\prime}, t_{q}^{\prime}\right)$ is a further eigenfunction for $L^{\prime}$, then $\hat{\Psi}^{\prime}\left(\hat{x}^{\prime}, \hat{t}_{q}^{\prime}\right)=\Phi_{x^{\prime}}^{\prime-1}\left(\Phi_{x^{\prime}}^{\prime} \Psi^{\prime}-\Phi^{\prime} \Psi_{x^{\prime}}^{\prime}\right)$ is an eigenfunction for $\hat{L}^{\prime}$, that is, $\hat{\Psi}_{\hat{t}_{q}}^{\prime}=\hat{P}_{\geq 2}^{\prime}$ $\left(\hat{L}^{\prime q}\right) \hat{\Psi}^{\prime}$. Here, $P_{\geq 2}^{\prime}$ and $\hat{P}_{\geq 2}^{\prime}$ are the projections associated with the differential symbols of the variables $x^{\prime}$ and $\hat{x}^{\prime}$, respectively.

These transformations encode auto-Bäcklund transformations of the isospectral equations for the Lax operators. For the $2+1$-equations described by the Lax hierarchies the transformations are worked out in Ref. [24]. Here we are mainly interested in the results for the $1+1$-dimensional integrable equations associated with Lax operators of the form (5.3).

The case $k=0$.

Starting with operators of the form $(5.3, k=0)$, we use an eigenfunction $\Phi$ satisfying $L \Phi=\lambda \Phi$. The gauge transformed operator is of the form

$$
\hat{L}=\Phi \partial \Phi^{-1} L \Phi \partial^{-1} \Phi^{-1}=\partial^{N}+\left(u_{N-2}+N \ln (\Phi)_{x x}\right) \partial^{N-2}+\cdots+(. .) \partial+(. .) .
$$

No integration symbols will turn up in $\hat{L}$, because they would be generated only by the zero order term of the operator $\Phi^{-1} L \Phi$. This term, however, 
is just the constant $\lambda$, as we have assumed $L \Phi=\lambda \Phi$. Identifying $\hat{L}=\Sigma \hat{u}_{i} \partial^{i}$, i.e. $\hat{u}_{N-2}=u_{N-2}+N \ln (\Phi)_{x x}$ etc., one finds a transformation from $\left(u_{N-2}, . ., u_{0}\right)$ to $\left(\hat{u}_{N-2}, . ., \hat{u}_{0}\right)$ in terms of the eigenfunction $\Phi$. Using $L \Phi=\lambda \Phi$ one eliminates $\Phi$ from this transformation and obtains the spatial part of an auto-Bäcklund transformation for the isospectral hierarchy associated with the Lax operator $\mathbb{L}$.

As simplest example, we again look at the Schrödinger operator $L=\partial^{2}+u$ for the $\mathbb{K d V}$ hierarchy. With $\hat{L}=\partial^{2}+\hat{u}$ the gauge transformation translates to

$$
\hat{u}=u+2 \ln (\Phi)_{x x}, \quad \Phi_{x x}+u \Phi=\lambda \Phi
$$

Eliminating $\Phi$ one obtains the standard auto-Bäcklund transformation

$$
\frac{\hat{u}+u}{2}=\lambda-\frac{1}{4}\left(D^{-1}(\hat{u}-u)\right)^{2}
$$

for the $\mathrm{KdV}$ hierarchy.

The case $R=1$.

Starting with operators of the form $(5.3, k=1)$, we use an eigenfunction $\tilde{\Phi}$ satisfying $\tilde{L} \tilde{\Phi}=\mu \widetilde{\Phi}$. The gauge transformed operator is of the form

$$
\tilde{\widetilde{L}}=\widetilde{\Phi}_{x}^{-1} \tilde{\Phi}^{2} \partial \tilde{\Phi}^{-1} \tilde{L} \tilde{\Phi} \partial^{-1} \tilde{\Phi}_{x} \tilde{\Phi}^{-2}=\partial^{N}+\left(v_{N-1}+N \frac{\ln (\tilde{\Phi})_{x x}}{\ln (\tilde{\Phi})_{x}}\right) \partial^{N-1}+. .+(. .) \partial+\lambda .
$$

Here no integration symbols will turn up in $\hat{\tilde{L}}$, because they would be generated only by the zero order term of the operator $\tilde{\Phi}^{-1} \tilde{L} \tilde{\Phi}$. This term, however, is just the constant $\mu$, as we have assumed $\widetilde{L} \widetilde{\Phi}=\mu \widetilde{\Phi}$. Hence, the zero order of $\hat{\tilde{L}}=\Sigma \hat{v}_{i} \partial^{i}$ can be calculated from the action of $\hat{\tilde{L}}$ on the constant function 1 . With $\left(\widetilde{\Phi}_{x} \widetilde{\Phi}^{-2}\right) 1=-\left(\partial \widetilde{\Phi}^{-1}\right) 1$ it is readily verified that the zero order term of $\tilde{\tilde{L}}$ coincides with the zero order term of $\tilde{L}$, that is, one finds $\hat{v}_{0}=\lambda$. Identifying $\hat{v}_{N-1}=v_{N-1}+N \frac{\ln (\Phi)_{x x}}{\ln (\Phi)_{x}}$ etc., one finds a transformation from $\left(v_{N-1}, . ., v_{1}\right)$ to $\left(\hat{v}_{N-1}, \ldots, \hat{v}_{1}\right)$ in terms of the eigenfunction $\widetilde{\Phi}$. Using $\widetilde{L} \tilde{\Phi}=\mu \widetilde{\Phi}$ one eliminates $\tilde{\Phi}$ from this transformation and obtains the spatial part of an auto-Bäcklund transformation for the isospectral hierarchy associated with the Lax operator $\tilde{L}$. 
As simplest example, we consider the second order operator $\widetilde{L}=\partial^{2}+2 v \partial$ $+\lambda$ for the modified $\mathrm{KdV}$ hierarchy. With $\tilde{\widetilde{L}}=\partial^{2}+2 \hat{v} \partial+\lambda$ the gauge transformation translates to

$$
\hat{v}=v+\frac{\ln (\widetilde{\Phi})_{x x}}{\ln (\widetilde{\Phi})_{x}}, \quad \widetilde{\Phi}_{x x}+2 v \widetilde{\Phi}_{x}=(\mu-\lambda) \widetilde{\Phi}
$$

Introducing $a=(\mu-\lambda)^{-\frac{1}{2}} \ln (\widetilde{\Phi})_{x}$ one obtains the equivalent relations

$$
\hat{v}-v=\frac{a_{x}}{a}, \quad \hat{v}+v=(\mu-\lambda)^{\frac{1}{2}}\left(\frac{1}{a}-a\right) .
$$

Integration of the first equation yields the well-known auto-Bäcklund transformation

$$
\hat{v}+v=2(\mu-\lambda)^{\frac{1}{2}} \sinh \left(\int^{x}(v-\hat{v})\right)
$$

shared by the modified $\mathrm{KdV}$ and the sinh-Gordon equation.

The case $k=2$.

Starting with operators of the form $(5.3, k=2)$, we use an eigenfunction $\Phi^{\prime}$ satisfying $L^{\prime} \Phi^{\prime}=\mu \Phi^{\prime}$. The linked operator is of the form

$$
\begin{aligned}
\hat{L}^{\prime} & =\Phi_{x^{\prime}}^{\prime-1} \Phi^{\prime 2} \partial^{\prime} \Phi^{\prime-1} L^{\prime} \Phi^{\prime} \partial^{\prime-1} \Phi_{x^{\prime}}^{\prime} \Phi^{\prime-2} \\
& =w_{N}\left(\frac{\Phi^{\prime} \Phi_{x^{\prime} x^{\prime}}^{\prime}}{\Phi_{x^{\prime}}^{\prime 2}}\right)^{N} \hat{\partial}^{\prime N}+\cdots+(. .) \hat{\partial}^{\prime 2}+\lambda_{1} \hat{x}^{\prime} \hat{\partial}^{\prime}+\lambda_{2},
\end{aligned}
$$

where $\hat{\partial}^{\prime}$ is the differential symbol associated with the new variable $\hat{x}^{\prime}=$ $x^{\prime}-\Phi_{x^{\prime}}^{\prime-1} \Phi^{\prime}$. The form of the first and zero order coefficients are verified with arguments similar to those of the case $k=1$. In particular, the first order coefficient of $\hat{L}^{\prime}$ is obtained by letting $\hat{L}^{\prime}$ act on $\hat{x}^{\prime}$. Hence, the transformation leaves the form $(5.3, k=2)$ of the Lax operators invariant. Identifying $\hat{L}^{\prime}=\Sigma \hat{w}_{i} \hat{\partial}^{\prime i}$, i.e. $\hat{w}_{N}=w_{N}\left(\Phi_{x^{\prime}}^{\prime-2} \Phi^{\prime} \Phi_{x^{\prime} x^{\prime}}^{\prime}\right)^{N}$ etc., one finds a transformation from $\left(w_{N}, . ., w_{2}\right)$ to $\left(\hat{w}_{N}, \ldots, \hat{w}_{2}\right)$ in terms of the eigenfunction $\Phi^{\prime}$. Using $L^{\prime} \Phi^{\prime}=\mu \Phi^{\prime}$ one eliminates $\Phi^{\prime}$ from this transformation and obtains the spatial 
part of an auto-Bäcklund transformation for the isospectral hierarchy associated with the Lax operator $L^{\prime}$.

As simplest example, we consider the operator $L^{\prime}=w^{2} \partial^{\prime 2}+\lambda_{1} x^{\prime} \partial^{\prime}+\lambda_{2}$ for the Dym hierarchy. With $\hat{L}^{\prime}=\hat{w}^{2} \hat{\partial}^{\prime 2}+\lambda_{1} \hat{x}^{\prime} \hat{\partial}^{\prime}+\lambda_{2}$ the reciprocal transformation translates to

$$
\hat{x}^{\prime}=x^{\prime}-\frac{\Phi^{\prime}}{\Phi_{x^{\prime}}^{\prime}}, \quad \hat{w}=w \frac{\Phi^{\prime} \Phi_{x^{\prime} x^{\prime}}^{\prime}}{\Phi_{x^{\prime}}^{\prime 2}}, \quad w^{2} \Phi_{x^{\prime} x^{\prime}}^{\prime}+\lambda_{1} x^{\prime} \Phi_{x^{\prime}}^{\prime}=\left(\mu-\lambda_{2}\right) \Phi^{\prime}
$$

Eliminating $\Phi^{\prime}$ one obtains the reciprocal auto-Bäcklund transformation for the Dym hierarchy. In particular, for the case $\lambda_{1,2}=0$ one uses $w \hat{w}=\mu \Phi_{x^{\prime}}^{\prime-2} \Phi^{\prime 2}$ and obtains

$$
\frac{\partial \hat{x}^{\prime}}{\partial x^{\prime}}=\frac{\hat{w}}{w}, \quad(\sqrt{w \hat{w}})_{x^{\prime}}=\sqrt{\mu}\left(1-\frac{\hat{w}}{w}\right)
$$

For each equation of the Dym hierarchy the corresponding time evolution $\Phi_{t_{q}^{\prime}}^{\prime}=P_{\geq 2}^{\prime}\left(L^{\prime q}\right) \Phi^{\prime}$ of the eigenfunction yields the time dependence of the transformation. For the Dym equation itself one has $q=\frac{3}{2}$, so that for $\lambda_{1,2}=0$

$$
\Phi_{t^{\prime}}^{\prime}=w^{3} \Phi_{x^{\prime} x^{\prime} x^{\prime}}^{\prime}+\frac{3}{2} w^{2} w_{x^{\prime}} \Phi_{x^{\prime} x^{\prime}}^{\prime}=\mu\left(w \Phi_{x^{\prime}}^{\prime}-\frac{1}{2} w_{x^{\prime}} \Phi^{\prime}\right)
$$

leads to

$$
\frac{\partial \hat{x}^{\prime}}{\partial t^{\prime}}=\frac{\partial}{\partial t^{\prime}}\left(x^{\prime}-\frac{\Phi^{\prime}}{\Phi_{x^{\prime}}^{\prime}}\right)=\mu(\hat{w}-w)-\frac{1}{2} \hat{w} w w_{x^{\prime} x^{\prime}}+w_{x^{\prime}} \sqrt{\mu w \hat{w}}
$$

As shown in Ref. [24] the auto-Bäcklund transformations for the cases $k=1$ and $k=2$ are compositions of more "elementary" Bäcklund transformations, which again may be described as gauge transformations on the Lax operators. For the case $k=1$ one has

Theorem 3. (gauges $k=\mathbb{1} \rightarrow k=1$ ) Let $\tilde{L} \in g$ satisfy the hierarchy of Lax equations $\frac{d}{d t_{q}} \tilde{L}=\left[P_{\geq 1}\left(\widetilde{L}^{q}\right), \tilde{L}\right]$. Let $\widetilde{\Phi}$ and $\tilde{\Psi}$ be two eigenfunctions of this hierarchy. Then 


$$
\begin{aligned}
\text { i) } \tilde{L}_{i}=\widetilde{\Phi}^{-1} \tilde{L} \tilde{\Phi}, \widetilde{\Psi}_{i}=\widetilde{\Phi}^{-1} \widetilde{\Psi} \\
\text { and } \quad \text { ii) } \tilde{L}_{i i}=\widetilde{\Phi}_{x}^{-1} \partial \tilde{L} \partial^{-1} \widetilde{\Phi}_{x}, \widetilde{\Psi}_{i i}=\widetilde{\Phi}_{x}^{-1} \widetilde{\Psi}_{x}
\end{aligned}
$$

both satisfy the $k=1$ hierarchy and $\tilde{\Psi}_{i}$ and $\tilde{\mathbb{\Psi}}_{i i}$ are eigenfunctions for $\tilde{L}_{i}$ and $\tilde{L}_{i i}$, respectively.

Assumptions such as $\tilde{\Phi} \neq 0, \widetilde{\Phi}_{x} \neq 0$ are required to perform these transformations. The corresponding auto-Bäcklund transformations in tems of the fields $\left(v_{N-1}, . ., v_{1}\right)$ parametrizing the Lax operators $(5.3, k=1)$ can be extracted as described before. In particular, for $\tilde{L}=\partial^{2}+2 v \partial+\lambda$, i.e. modified $\mathrm{KdV}$ hierarchy, one uses an eigenfunction $\widetilde{\Phi}$ satisfying $\tilde{L} \widetilde{\Phi}=\mu \widetilde{\Phi}$ and finds

$$
\begin{aligned}
& \tilde{L}_{i}=\partial^{2}+2 v_{i} \partial+\mu, \quad v_{i}=v+\frac{\widetilde{\Phi}_{x}}{\widetilde{\Phi}} \\
& \tilde{L}_{i i}=\partial^{2}+2 v_{i i} \partial+\mu, \quad v_{i i}=v+\frac{\widetilde{\Phi}_{x x}}{\widetilde{\Phi}_{x}}
\end{aligned}
$$

Elimination of $\tilde{\Phi}$ using $\widetilde{\Phi}_{x x}+2 v \widetilde{\Phi}_{x}=(\mu-\lambda) \widetilde{\Phi}$ leads to

$$
\left(v_{i}-v\right)_{x}+\left(v_{i}-v\right)\left(v_{i}+v\right)=\mu-\lambda, \quad\left(v_{i i}+v\right)_{x}+\left(v_{i i}-v\right)\left(v_{i i}+v\right)=\mu-\lambda
$$

as the spatial parts of these Bäcklund transformations. We note, that in contrast to the transformation of Theorem 2 , the zero-order term $\lambda$ in $\tilde{L}$ has changed to $\mu$ in $\widetilde{L}_{i}$ and $\tilde{L}_{i i}$. Hence, in general, a solution of the modified $\mathrm{KdV}$ (2.37) with a given $\lambda$ will be mapped to a solution with a different value for $\lambda$. For the special choice $\lambda=\mu$ there are the obvious solutions $v_{i}=v$ and $v_{i i}=-v$ as special cases of these transformations. As we shall see soon, the composition of the two transformations gives rise to the transformation (5.19).

Also for the case $k=2$ the auto-Bäcklund transformations of Theorem 2 may be decomposed into a sequence of elementary Bäcklund transformations given by

Theorem 4. (links $k=2 \rightarrow k=2)$ Let $L^{\prime}=L^{\prime}\left(x^{\prime}, t_{q}^{\prime}\right)$ satisfy $\frac{d}{d t_{q}^{\prime}} L^{\prime}=$ $\left[P_{\geq 2}^{\prime}\left(L^{\prime q}\right), L^{\prime}\right]$. Let $\Phi^{\prime}\left(x^{\prime}, t_{q}^{\prime}\right)$ and $\Psi^{\prime}\left(x^{\prime}, t_{q}^{\prime}\right)$ be two eigenfunctions of this hierarchy. Consider the following transformations:

i) $\hat{x}^{\prime}=\Phi^{\prime}\left(x^{\prime}, t_{q}^{\prime}\right), \hat{t}_{q}^{\prime}=t_{q}^{\prime}, \hat{L}^{\prime}\left(\hat{x}^{\prime}, \hat{t}_{q}^{\prime}\right)=L^{\prime}\left(x^{\prime}, t_{q}^{\prime}\right) \quad, \hat{\Psi}^{\prime}\left(\hat{x}^{\prime}, \hat{t}_{q}^{\prime}\right)=\Psi^{\prime}\left(x^{\prime}, t_{q}^{\prime}\right)$, 
ii) $\hat{x}^{\prime}=x^{\prime-1} \quad, \hat{t}_{q}^{\prime}=t_{q}^{\prime}, \hat{L}^{\prime}\left(\hat{x}^{\prime}, \hat{t}_{q}^{\prime}\right)=x^{\prime-1} L^{\prime}\left(x^{\prime}, t_{q}^{\prime}\right) x^{\prime}, \hat{\Psi}^{\prime}\left(\hat{x}^{\prime}, \hat{t}_{q}^{\prime}\right)=x^{\prime-1} \Psi^{\prime}\left(x^{\prime}, t_{q}^{\prime}\right)$,

iii) $\hat{x}^{\prime}=\mathbb{\Phi}_{x^{\prime}}^{\prime}\left(x^{\prime}, t_{q}^{\prime}\right), \hat{t}_{q}^{\prime}=t_{q}^{\prime}, \hat{L}^{\prime}\left(\hat{x}^{\prime}, \hat{t}_{q}^{\prime}\right)=\partial^{\prime} \mathbb{L}^{\prime}\left(x^{\prime}, t_{q}^{\prime}\right) \partial^{\prime-1}, \hat{\Psi}^{\prime}\left(\hat{x}^{\prime}, \hat{t}_{q}^{\prime}\right)=\Psi_{x^{\prime}}^{\prime}\left(x^{\prime}, \hat{t}_{q}^{\prime}\right)$.

Then, in all cases, $\hat{L}^{\prime}$ satisfies the $k=2$ hievarchy $\frac{d}{d \hat{t}_{q}^{\prime}} \hat{L}^{\prime}=\left[P_{\geq 2}^{\prime}\left(\hat{L}^{\prime q}\right), \hat{L}^{\prime}\right]$ and $\hat{\Psi}^{\prime}$ is an eigenfunction of $\hat{\mathbb{L}}^{\prime}$, that is, $\hat{\Psi}_{\hat{t}_{q}^{\prime}}^{\prime}=\hat{P}_{\geq 2}^{\prime}\left(\hat{\mathbb{L}}^{\prime q}\right) \hat{\Psi}^{\prime}$.

The corresponding Bäcklund transformations in terms of the fields $\left(w_{N-1}, \ldots, w_{1}\right)$ parametrizing the $\operatorname{Lax}$ operators $(5.3, k=2)$ can be extracted as described before. In particular, for $L^{\prime}=w^{2} \partial^{\prime 2}+\lambda_{1} x^{\prime} \partial^{\prime}+\lambda_{2}$, i.e. the Dym hierarchy, one uses an eigenfunction $\Phi^{\prime}$ satisfying $L^{\prime} \Phi^{\prime}=\mu \Phi^{\prime}$ and finds
i) $\hat{L}^{\prime}=w_{i}^{2} \hat{\partial}^{\prime 2}+\left(\mu-\lambda_{2}\right) \hat{x}^{\prime} \hat{\partial}^{\prime}+\lambda_{2}, \quad w_{i}=w \Phi_{x^{\prime}}^{\prime}$,
ii) $\hat{L}^{\prime}=w_{i i}^{2} \partial^{\prime 2}+\lambda_{1} \hat{x}^{\prime} \hat{\partial}^{\prime}+\lambda_{1}+\lambda_{2}, \quad w_{i i}=x^{\prime-2} w$,
iii) $\hat{L}^{\prime}=w_{i i i}^{2} \partial^{\prime 2}+\left(\mu-\lambda_{1}-\lambda_{2}\right) \hat{x}^{\prime} \hat{\partial}^{\prime}+\lambda_{1}+\lambda_{2}, \quad w_{i i i}=w \Phi_{x^{\prime} x^{\prime}}^{\prime}$.

Hence, these transformations change the values of the constant parameters $\lambda_{1.2}$ in $L^{\prime}$ and solutions of the Dym hierarchy with given values of the $\lambda^{\prime}$ 's are mapped to solutions with different values. As before, elimination of $\Phi^{\prime}$ in the transformations i) and iii) yield reciprocal Bäcklund transformations involving the fields $w, w_{i}$, and $w_{i i i}$. As observed in Ref. [24], Theorem 4 encapsulates the well known invariances of the Dym equation triggered by a Moebius transformation of the space variable. As we will see, a composition of the three transformations i)-iii) leads to the invariance given by Theorem 2 .

We summarize the various results of the theorems by introducing the notation

$$
\begin{aligned}
& \mathbb{B}_{0 \rightarrow 0}(\Phi): \quad L \rightarrow \Phi \partial \Phi^{-1} L \Phi \partial^{-1} \Phi^{-1}, \Psi \rightarrow \Phi\left(\Phi^{-1} \Psi\right)_{x}, \\
& \mathbb{B}_{0 \rightarrow 1}(\Phi): \quad \mathbb{L} \rightarrow \Phi^{-1} L \Phi, \Psi \rightarrow \Phi^{-1} \Psi, \\
& \mathbb{B}_{1 \rightarrow 1}^{i}(\widetilde{\Phi}): \quad \widetilde{L} \rightarrow \widetilde{\Phi}^{-1} \tilde{L} \widetilde{\Phi}, \widetilde{\Psi} \rightarrow \widetilde{\Phi}^{-1} \widetilde{\Psi}, \\
& \mathbb{B}_{1 \rightarrow 1}^{i i}(\widetilde{\Phi}): \quad \tilde{L} \rightarrow \widetilde{\Phi}_{x}^{-1} \partial \widetilde{L} \partial^{-1} \widetilde{\Phi}_{x}, \tilde{\Psi} \rightarrow \widetilde{\Phi}_{x}^{-1} \tilde{\Psi}_{x}, \\
& \mathbb{B}_{1 \rightarrow 1}(\widetilde{\Phi}): \quad \widetilde{L} \rightarrow \widetilde{\Phi}_{x}^{-1} \widetilde{\Phi}^{2} \partial \widetilde{\Phi}^{-1} \tilde{L} \tilde{\Phi} \partial^{-1} \widetilde{\Phi}_{x} \widetilde{\Phi}^{-2}, \tilde{\Psi} \rightarrow \widetilde{\Phi}_{x}^{-1}\left(\widetilde{\Phi}_{x} \widetilde{\Psi}-\tilde{\Psi} x\right) \text {, } \\
& \mathbb{B}_{1 \rightarrow 2}(\widetilde{\Phi}): \quad \tilde{L} \rightarrow L^{\prime}=\tilde{L}, \tilde{\Psi} \rightarrow \Psi^{\prime}=\tilde{\Psi}, x \rightarrow x^{\prime}=\widetilde{\Phi}, \\
& \mathbb{B}_{2 \rightarrow 2}^{i}\left(\Phi^{\prime}\right): \quad L^{\prime} \rightarrow \hat{L}^{\prime}=L^{\prime}, \Psi^{\prime} \rightarrow \hat{\Psi}^{\prime}=\Psi^{\prime}, x^{\prime} \rightarrow \hat{x}^{\prime}=\Phi^{\prime}, \\
& \mathbb{B}_{2 \rightarrow 2}^{i i}: \quad L^{\prime} \rightarrow \hat{L}^{\prime}=x^{\prime-1} L^{\prime} x^{\prime}, \Psi^{\prime} \rightarrow \Psi^{\prime}=x^{\prime-1} \Psi^{\prime}, x^{\prime} \rightarrow \hat{x}^{\prime}=x^{\prime-1} \text {, } \\
& \mathbb{B}_{2 \rightarrow 2}^{i i i}\left(\Phi^{\prime}\right): \quad L^{\prime} \rightarrow \hat{L}^{\prime}=\partial^{\prime} L^{\prime} \partial^{\prime-1}, \Psi^{\prime} \rightarrow \hat{\Psi}^{\prime}=\Psi_{x^{\prime}}^{\prime}, x^{\prime} \rightarrow \hat{x}^{\prime}=\Phi_{x^{\prime}}^{\prime} \text {, } \\
& \mathbb{B}_{2 \rightarrow 2}\left(\Phi^{\prime}\right): \quad L^{\prime} \rightarrow \hat{L}^{\prime}=\Phi_{x^{\prime}}^{\prime-1} \Phi^{\prime 2} \partial^{\prime} \Phi^{\prime-1} L^{\prime} \Phi^{\prime} \partial^{\prime-1} \Phi_{x^{\prime}}^{\prime} \Phi^{\prime-2} \text {, } \\
& \Psi^{\prime} \rightarrow \Psi^{\prime}=\Phi_{x^{\prime}}^{\prime-1}\left(\Phi_{x^{\prime}}^{\prime} \Psi^{\prime}-\Psi_{x^{\prime}}^{\prime} \Phi^{\prime}\right), x^{\prime} \rightarrow \hat{x}^{\prime}=x^{\prime}-\Phi_{x^{\prime}}^{\prime-1} \Phi^{\prime} .
\end{aligned}
$$


Here the fairly obvious notation $\boldsymbol{B}_{0 \rightarrow 0}, \boldsymbol{B}_{0 \rightarrow 1}$ etc. is used for the transformations linking $k=0 \rightarrow k=0, k=0 \rightarrow k=1$ etc. The transformations $\boldsymbol{B}_{1 \rightarrow 1}^{i, i i}, \boldsymbol{B}_{2 \rightarrow 2}^{i-i i i}$ are the elementary Bäcklund transformations of Theorems 3 and 4 .

So far we have not made any use of the fact that the theorems actually provide additional information in the spirit of Darboux theorems. A first eigenfunction for the Lax operator can be used to generate a new solution of either the same equation or to the Miura- or reciprocally linked equation. Now, if a further eigenfunction of the original Lax operator is known, the theorems show, how this eigenfunction has to be transformed into an eigenfunction of the transformed operator. Hence, one can start from there to perform an additional transformation using this new eigenfunction. It turns out that such a process leads to various connections between these transformations. In fact, we will finally see with the diagrams following later on that all transformations above form a very logical pattern.

We remark that the hierarchies of the cases $k=1$ and $k=2$ have "trivial" eigenfunctions, as the required time evolutions $\Phi_{t_{q}}=P_{\geq k}\left(L^{q}\right) \Phi, k=1,2$ involve only derivatives of first and higher orders. Namely, for $k=1$ the constant function $\Phi\left(x, t_{q}\right)=1$ and for $k=2$ the functions $\Phi\left(x, t_{q}\right)=1, \Phi\left(x, t_{q}\right)=x$ will be eigenfunctions, trivially.

It was claimed before that the transformations $\boldsymbol{B}_{1 \rightarrow 1}$ and $\boldsymbol{B}_{2 \rightarrow 2}$ are compositions of the "elementary" transformations given by Theorems 3 and 4 . Indeed, considering the case $k=1$, it is readily verified that

$$
B_{1 \rightarrow 1}(\widetilde{\Phi})=B_{1 \rightarrow 1}^{i i}\left(\widetilde{\Phi}^{-1}\right) B_{1 \rightarrow 1}^{i}(\widetilde{\Phi})
$$

where $\widetilde{\Phi}^{-1}=B_{1 \rightarrow 1}^{i}(\tilde{\Phi}) 1$ is the eigenfunction of the operator $B_{1 \rightarrow 1}^{i}(\tilde{\Phi}) \tilde{L}$ originating from the trivial eigenfunction 1 of the operator $\widetilde{L}$.

It was already remarked in Ref. [24] that also the auto-Bäcklund transformations of the different classes are related. First, looking at the classes $k=0$ and $k=1$, one easily verifies the identities

$$
\begin{gathered}
\boldsymbol{B}_{0 \rightarrow 1}(\Phi)=\mathbb{B}_{1 \rightarrow 1}^{i}\left(\boldsymbol{B}_{0 \rightarrow 1}(\Psi) \Phi\right) \boldsymbol{B}_{0 \rightarrow 1}(\Psi) \\
\boldsymbol{B}_{0 \rightarrow 1}\left(B_{0 \rightarrow 0}(\Phi) \Psi\right) \boldsymbol{B}_{0 \rightarrow 0}(\Phi)=B_{1 \rightarrow 1}^{i i}\left(B_{0 \rightarrow 1}(\Phi) \Psi\right) \boldsymbol{B}_{0 \rightarrow 1}(\Phi)
\end{gathered}
$$

for arbitrary functions $\Phi$ and $\Psi$. As a consequence, one also has

$$
B_{0 \rightarrow 1}\left(B_{0 \rightarrow 0}(\Phi) \Psi\right) B_{0 \rightarrow 0}(\Phi)=B_{1 \rightarrow 1}\left(B_{0 \rightarrow 1}(\Psi) \Phi\right) B_{0 \rightarrow 1}(\Psi)
$$


These relations are conveniently presented in a commuting diagram. In Diagram 1 one starts with the operator $L$ satisfying the $k=0$ hierarchy. If two eigenfunctions $\Phi$ and $\Psi$ are given, then we use $\Phi$ to generate a further solution $\hat{L}$ of the $k=0$ hierarchy, and $\Psi$ is transformed to an eigenfunction $\hat{\Psi}$ for $\hat{L}$. In this transformation the original eigenfunction $\Phi$ for $L$ is "used up", that is, it is mapped to the trivial eigenfunction $B_{0 \rightarrow 0}(\Phi): \Phi \rightarrow 0$ for $\hat{L}$. Now one uses the $\Psi$ 's to generate solutions $\tilde{L}$ and $\tilde{\tilde{L}}$ of the $k=1$ hierarchy, starting from $L$ and $\hat{L}$, respectively. In this process, the eigenfunctions used for the transformations trivialize to constant functions. However, starting from $L$ one can map the eigenfunction $\Phi$ to an eigenfunction $\widetilde{\Phi}$ for $\tilde{L}$. Using $B_{1 \rightarrow 1}$ with this eigenfunction one ends up with the operator $\hat{\tilde{L}}$ already constructed from $\hat{L}$. As $B_{1 \rightarrow 1}$ decomposes into two elementary transformations $\boldsymbol{B}_{1 \rightarrow 1}^{i}$ and $\boldsymbol{B}_{1 \rightarrow 1}^{i i}$, an "intermediate" solution $\tilde{l}=\boldsymbol{B}_{1 \rightarrow 1}^{i}(\tilde{\Phi}) \tilde{L}$ is generated on the way from $\tilde{L}$ to $\tilde{\tilde{L}}$. As shown in the diagram, even this intermediate $\tilde{l}$ is directly linked to the original solution $L$ of the $k=0$ hierarchy via the original eigenfunction $\Phi$.

Further, it is easy to check that the Bianchi diagrams of the Bäcklund transformations encoded in $\boldsymbol{B}_{0 \rightarrow 0}$ and $\boldsymbol{B}_{1 \rightarrow 1}$ commute, that is, one finds the identities

$$
\begin{aligned}
& B_{0 \rightarrow 0}\left(B_{0 \rightarrow 0}\left(\Phi_{1}\right) \Phi_{2}\right) B_{0 \rightarrow 0}\left(\Phi_{1}\right)=B_{0 \rightarrow 0}\left(B_{0 \rightarrow 0}\left(\Phi_{2}\right) \Phi_{1}\right) B_{0 \rightarrow 0}\left(\Phi_{2}\right) \\
& B_{1 \rightarrow 1}\left(B_{1 \rightarrow 1}\left(\widetilde{\Phi}_{1}\right) \widetilde{\Phi}_{2}\right) B_{1 \rightarrow 1}\left(\widetilde{\Phi}_{1}\right)=B_{1 \rightarrow 1}\left(B_{1 \rightarrow 1}\left(\widetilde{\Phi}_{2}\right) \widetilde{\Phi}_{1}\right) B_{1 \rightarrow 1}\left(\widetilde{\Phi}_{2}\right)
\end{aligned}
$$

for arbitrary functions $\Phi_{1,2}, \widetilde{\Phi}_{1,2}$. As a consequence of the relations given

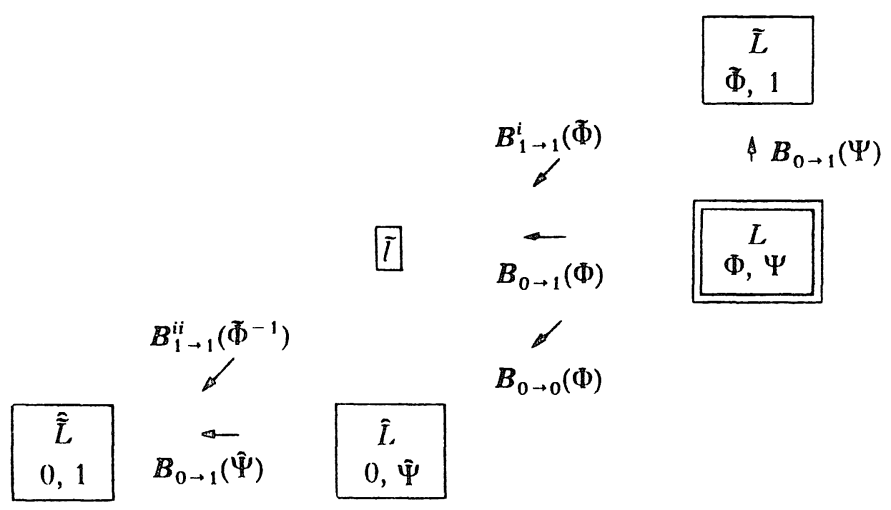

Diagram 1 


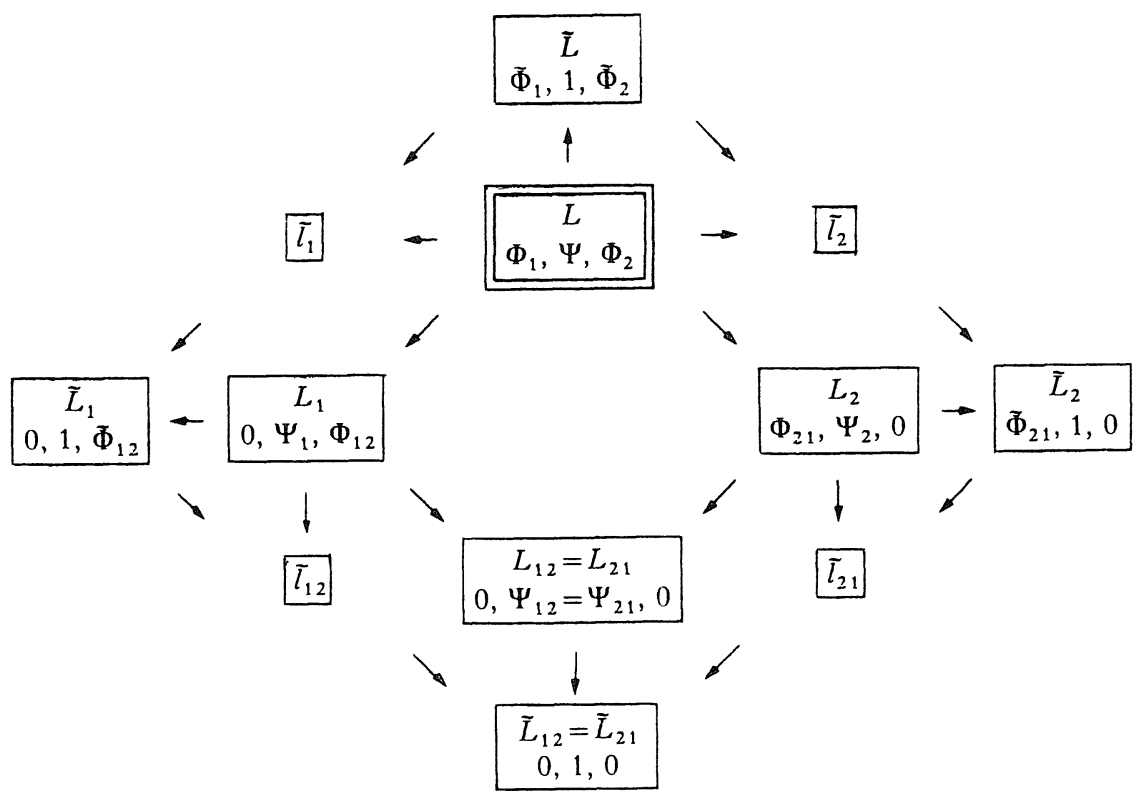

Diagram 2

by Diagram 1, the Bianchi diagrams for $\boldsymbol{B}_{0 \rightarrow 0}$ and $\boldsymbol{B}_{1 \rightarrow 1}$ can be summarized in a single diagram. In Diagram 2 the inner rhomb refers to the $k=0$ solutions and the outer rhomb refers to the $k=1$ solutions. The upper left part of Diagram 2 may be identified with Diagram 1. In fact, the Bianchi Diagram 2 is constructed from 4 copies of Diagram 1 .

In this diagram one has

$$
\begin{aligned}
& \tilde{L}=B_{0 \rightarrow 1}(\Psi) L, \tilde{\Phi}_{1}=B_{0 \rightarrow 1}(\Psi) \Phi_{1}=\Psi^{-1} \Phi_{1}, \tilde{l}=B_{1 \rightarrow 1}^{i}\left(\tilde{\Phi}_{1}\right) \tilde{L}=B_{0 \rightarrow 1}\left(\Phi_{1}\right) L, \\
& L_{1}=B_{0 \rightarrow 0}\left(\Phi_{1}\right) L, \Psi_{1}=B_{0 \rightarrow 0}\left(\Phi_{1}\right) \Psi=-\Phi_{1}^{-1} W\left(\Phi_{1}, \Psi\right), \Phi_{12}=B_{0 \rightarrow 0}\left(\Phi_{1}\right) \Phi_{2} \\
& =-\Phi_{1}^{-1} W\left(\Phi_{1}, \Phi_{2}\right) \text {, } \\
& \tilde{L}_{1}=B_{1 \rightarrow 1}^{i i}\left(\widetilde{\Phi}_{1}^{-1}\right) \tilde{l}_{1}=B_{1 \rightarrow 1}\left(\widetilde{\Phi}_{1}\right) \tilde{L}=B_{0 \rightarrow 1}\left(\Psi_{1}\right) L_{1} \text {, } \\
& \widetilde{\Phi}_{12}=B_{1 \rightarrow 1}\left(\tilde{\Phi}_{1}\right) \tilde{\Phi}_{2}=B_{0 \rightarrow 1}\left(\Psi_{1}\right) \Phi_{12}=\frac{W\left(\Phi_{1}, \Phi_{2}\right)}{W\left(\Phi_{1}, \Psi\right)}, \tilde{l}_{12}=B_{1 \rightarrow 1}^{i}\left(\widetilde{\Phi}_{12}\right) \tilde{L}_{1} \\
& =\boldsymbol{B}_{0 \rightarrow 1}\left(\Phi_{12}\right) L_{1} \\
& L_{12}=B_{0 \rightarrow 0}\left(\Phi_{12}\right) L_{1}, \Psi_{12}=B_{0 \rightarrow 0}\left(\Phi_{12}\right) \Psi_{1}=-\frac{W\left(\Phi_{1}, \Psi, \Phi_{2}\right)}{W\left(\Phi_{1}, \Phi_{2}\right)},
\end{aligned}
$$




$$
\tilde{L}_{12}=B_{1 \rightarrow 1}^{i i}\left(\tilde{\Phi}_{12}^{-1}\right) \tilde{l}_{12}=B_{1 \rightarrow 1}\left(\tilde{\Phi}_{12}\right) \tilde{L}_{1}=B_{0 \rightarrow 1}\left(\Psi_{12}\right) L_{12},
$$

and the remaining operators and eigenfunctions are given by the exchange of indices $1 \leftrightarrow 2$. The eigenfunctions are conveniently expressed in terms of Wronskian determinants $W$.

In Diagram 2 we have started with the operator $L$, equipped with three eigenfunctions $\Phi_{1,2}$ and $\Psi$. Using $\mathbb{B}_{0 \rightarrow 0}$ the two eigenfunctions $\Phi_{1,2}$ are used to generate the commuting Bianchi diagram via $L_{1}$ and $L_{2}$ to $L_{12}=L_{21}$. In each stage, the eigenfunction used for the gauge transformation is "used up" and trivializes. The third eigenfunction $\Psi$ for $L$ is carried through the transformations and yields eigenfunctions for $L_{1}, L_{2}$ and $L_{12}$. These $\Psi$ 's are then used with $\mathbb{B}_{0 \rightarrow 1}$ to generate solutions $\tilde{L}, \tilde{L}_{1}, \tilde{L}_{2}$ and $\tilde{L}_{12}$ of the $k=1$ hierarchy. They form the vertices of the commuting Bianchi diagram for the $k=1$ case: using $B_{1 \rightarrow 1}$ with the eigenfunctions $\widetilde{\Phi}_{1.2}$ obtained from the original eigenfunctions $\Phi_{1,2}$ of $L$, one finds the same solutions. As shown in Diagram 1 even the "intermediate" operators $\tilde{l}_{1}$, $\tilde{l}_{2}, \tilde{l}_{12}$ and $\tilde{l}_{21}$ generated from the elementary Bäcklund transformations of the class $k=1$ are related to the original operators satisfying the $k=0$ hierarchy. Thus, Diagram 1 summarizes the connections between the various gauge transformations related to the cases $k=0$ and $k=1$.

Similar relations can be established among the Bäcklund transformations connected with $k=1$ and $k=2$. First, we observe the transformation $\mathbb{B}_{2 \rightarrow 2}$ decomposes into a composition of the three elementary transformations of Theorem 4:

$$
B_{2 \rightarrow 2}\left(\Phi^{\prime}\right)=B_{2 \rightarrow 2}^{i i i}\left(\Phi^{\prime-1} x^{\prime}\right) B_{2 \rightarrow 2}^{i i} B_{2 \rightarrow 2}^{i}\left(\Phi^{\prime}\right)
$$

where $\Phi^{\prime-1} x^{\prime}=B_{2 \rightarrow 2}^{i i} B_{2 \rightarrow 2}^{i}\left(\Phi^{\prime}\right) x^{\prime}$ is the eigenfunction of the operator $B_{2 \rightarrow 2}^{i i} B_{2 \rightarrow 2}^{i}\left(\Phi^{\prime}\right) L^{\prime}$ originating from the trivial eigenfunction $x^{\prime}$ of the operator $L^{\prime}$. Indeed, we start with $L^{\prime}$ and the independent variable $x^{\prime}$, an eigenfunction $\Psi^{\prime}$ and the trivial eigenfunction $x^{\prime}$. Performing the first two steps one has

$$
\begin{aligned}
& B_{2 \rightarrow 2}^{i}\left(\Phi^{\prime}\right) \quad \mathbb{B}_{2 \rightarrow 2}^{i i} \\
& L^{\prime}, x^{\prime} \quad L_{i}^{\prime}=L, x_{i}^{\prime}=\Phi^{\prime} \quad L_{i i}^{\prime}=x_{i}^{\prime-1} L_{i}^{\prime} x_{1}^{\prime}=\Phi^{\prime-1} L^{\prime} \Phi^{\prime}, x_{i i}^{\prime}=x_{i}^{\prime-1}=\Phi^{\prime-1} \\
& \Psi^{\prime}, x^{\prime} \quad \rightarrow \quad \Psi_{i}^{\prime}=\Psi^{\prime}, x^{\prime} \quad \rightarrow \quad \Psi_{i i}^{\prime}=x_{i}^{\prime-1} \Psi_{i}^{\prime}=\Phi^{\prime-1} \Psi^{\prime}, x_{i}^{\prime-1} x^{\prime}=\Phi^{\prime-1} x^{\prime}
\end{aligned}
$$


Using the eigenfunction $\Phi^{\prime-1} x^{\prime}$ for $L_{i i}^{\prime}$, the final application of $B_{2 \rightarrow 2}^{i i i}$ leads to

$$
\begin{aligned}
& L_{i i i}^{\prime}=\partial_{x_{i i}^{\prime}} L_{i i}^{\prime} \partial_{x_{i i}^{\prime}}-1=\left(\frac{\partial x_{i i}^{\prime}}{\partial x^{\prime}}\right)^{-1} \partial^{\prime} L_{i i}^{\prime} \partial^{\prime}-1 \frac{\partial x_{i i}^{\prime}}{\partial x^{\prime}}=\Phi_{x^{\prime}}^{\prime-1} \Phi^{\prime 2} \partial^{\prime} \Phi^{\prime-1} L^{\prime} \Phi^{\prime} \partial^{\prime-1} \Phi_{x^{\prime}}^{\prime} \Phi^{\prime-2} \\
& x_{i i i}^{\prime}=\left(\Phi^{\prime-1} x^{\prime}\right)_{x_{i i}^{\prime}}=\left(\frac{\partial x_{i i}^{\prime}}{\partial x^{\prime}}\right)^{-1}\left(\Phi^{\prime-1} x^{\prime}\right)_{x^{\prime}}=\frac{\left(x^{\prime} / \Phi^{\prime}\right)_{x^{\prime}}}{\left(1 / \Phi^{\prime}\right)_{x^{\prime}}}=x^{\prime}-\Phi_{x^{\prime}}^{\prime-1} \Phi^{\prime} \\
& \Psi_{i i i}^{\prime}=\left(\Psi_{i i}^{\prime}\right)_{x_{i i}^{\prime}}=\left(\frac{\partial x_{i i}^{\prime}}{\partial x^{\prime}}\right)^{-1}\left(\Psi_{i i}^{\prime}\right)_{x^{\prime}}=\frac{\left(\Psi^{\prime} / \Phi^{\prime}\right)_{x^{\prime}}}{\left(1 / \Phi^{\prime}\right)_{x^{\prime}}}=\Phi_{x^{\prime}}^{\prime-1}\left(\Phi_{x^{\prime}}^{\prime} \Psi^{\prime}-\Psi_{x^{\prime}}^{\prime} \Phi^{\prime}\right)
\end{aligned}
$$

which are precisely the results of the transformation $\boldsymbol{B}_{\mathbf{2} \rightarrow 2}$.

For the pair $k=1, k=2$ one can establish relations similar to those given by $(5.29) /(5.30)$. They are summarized in the commuting Diagram 3 .

Here we start with the operator $\tilde{L}$ satisfying the $k=1$ hierarchy using the space variable $x$. An eigenfunction $\widetilde{\Phi}$ is used to generate the new solution $\hat{\tilde{L}}=B_{1 \rightarrow 1}(\tilde{\Phi}) \tilde{L}$. Using the elementary transformations $\boldsymbol{B}_{1 \rightarrow 1}^{i}, \boldsymbol{B}_{1 \rightarrow 1}^{i i}$ an intermediate solution $\tilde{l}=\boldsymbol{B}_{1 \rightarrow 1}^{i}(\widetilde{\Phi}) \tilde{L}$ is generated on the way. Using a further eigenfunction $\tilde{\Psi}$ one generates a solution $L^{\prime}$ of the $k=2$ hierarchy. Pushing $\tilde{\Phi}$ through this transformation one obtains an eigenfunction $\Phi^{\prime}=\tilde{\Phi}$ for $L^{\prime}$, which is used to generate a further solution $\hat{L}^{\prime}=B_{2 \rightarrow 2}\left(\Phi^{\prime}\right) L^{\prime}$ of the $k=2$

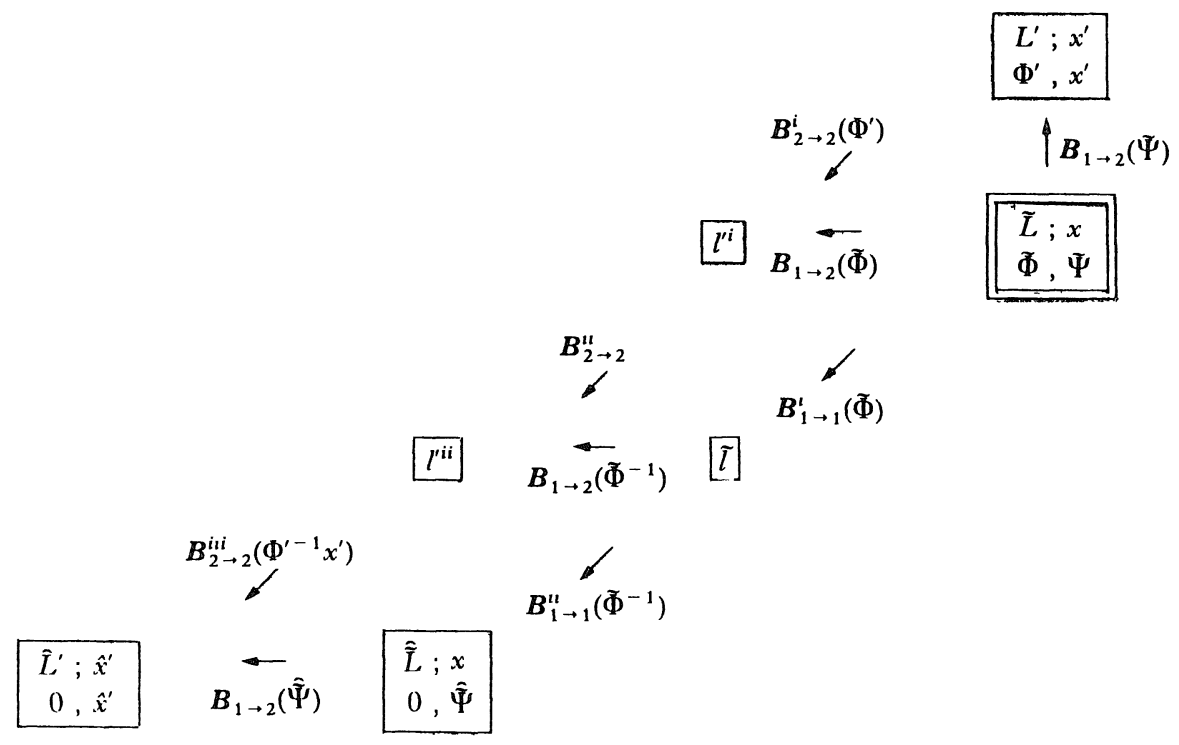

Diagram 3 
hierarchy. Again, intermediate solutions $l^{i}=\mathbb{B}_{2 \rightarrow 2}^{i}\left(\Phi^{\prime}\right) L^{\prime}$, and $l^{\prime i i}=B_{2 \rightarrow 2}^{i i} l^{i}$ are generated on the way, as $\boldsymbol{B}_{2 \rightarrow 2}$ decomposes into elementary transformations. The operator $\hat{L}^{\prime}$ can also be obtained from $\hat{\tilde{L}}$ via $B_{1 \rightarrow 2}$, using the eigenfunction $\hat{\Psi}=B_{1 \rightarrow 1}(\tilde{\Phi}) \tilde{\Psi}$. As shown in Diagram 3, also the intermediate solutions are related via $B_{1 \rightarrow 2}$.

It is proven in Ref. [24] that the Bianchi diagram for the class $k=2$ commutes, that is, in addition to (5.31) one has

$$
\mathbb{B}_{2 \rightarrow 2}\left(B_{2 \rightarrow 2}\left(\Phi_{1}^{\prime}\right) \Phi_{2}^{\prime}\right) B_{2 \rightarrow 2}\left(\Phi_{1}^{\prime}\right)=B_{2 \rightarrow 2}\left(B_{2 \rightarrow 2}\left(\Phi_{2}^{\prime}\right) \Phi_{1}^{\prime}\right) B_{2 \rightarrow 2}\left(\Phi_{2}^{\prime}\right)
$$

for arbitrary functions $\Phi_{1,2}^{\prime}$. Because of the relations given by Diagram 3 we can think of the Bianchi diagram for $\boldsymbol{B}_{1 \rightarrow 1}$ as sitting inside the Bianchi diagram for $\boldsymbol{B}_{2 \rightarrow 2}$. Using the independent variable $x$, we start with an operator $\tilde{L}$, now equipped with three eigenfunctions $\widetilde{\Phi}_{1,2}$ and $\widetilde{\Psi}$. As before, one uses $\widetilde{\Phi}_{1,2}$ to construct $\widetilde{L}_{1}, \widetilde{L}_{2}$ and, finally, $\widetilde{L}_{12}=\widetilde{L}_{21}$. Now, as one has an additional eigenfunction $\tilde{\Psi}$ of $\tilde{L}$, this eigenfunction is carried through the transformations yielding eigenfunctions $\widetilde{\Psi}_{1}$ for $\widetilde{L}_{1}, \widetilde{\Psi}_{2}$ for $\widetilde{L}_{2}$, and, finally, $\tilde{\Psi}_{12}=\widetilde{\Psi}_{21}$ for $\tilde{L}_{12}$. Each of these $\Psi$ 's can now be used via $\mathbb{B}_{1 \rightarrow 2}$ to generate solutions of the $k=2$ hierarchy, i.e., one has $L^{\prime}$ with the space variable $x^{\prime}=\tilde{\Psi}, L_{1}^{\prime}$ with $x_{1}^{\prime}=\tilde{\Psi}_{1}, L_{2}^{\prime}$ with $x_{2}^{\prime}=\tilde{\Psi}_{2}$, and, finally, $L_{12}^{\prime}=L_{21}^{\prime}$ with $x_{12}^{\prime}=\tilde{\Psi}_{12}$. In each transformation the $\widetilde{\Phi}$ 's are converted to new eigenfunctions of the class $k=2$ using the corresponding transformation. The operators $L^{\prime}, L_{1}^{\prime}, L_{2}^{\prime}$ and $L_{12}^{\prime}$ form the vertices of the commuting Bianchi Diagram for the $k=2$ class, when we regard $L^{\prime}$ to be equipped with the eigenfunctions $\Phi_{1}^{\prime}$ and $\Phi_{2}^{\prime}$ originating from the original eigenfunctions $\widetilde{\Phi}_{1}$ and $\widetilde{\Phi}_{2}$ for $\tilde{L}$. Hence, we may summarize the Bianchi diagrams for the classes $k=1$ and $k=2$ in a single picture given by Diagram 4 . The outer rhomb refers to the $k=2$ solutions, whereas the $k=1$ solutions form the rhomb sitting inside. Identifying the left upper quarter of this diagram with Diagram 3, one sees that the whole diagram really is built out of 4 copies of Diagram 3. Diagram 2 may be thought of a sitting inside this diagram, this is indicated by the innermost rhomb related to the $k=0$ case.

In this diagram one has

$$
\begin{aligned}
& L^{\prime}=B_{1 \rightarrow 2}(\tilde{\Psi}) \tilde{L}, l_{1}^{i}=\mathbb{B}_{2 \rightarrow 2}^{i}\left(\Phi_{1}^{\prime}\right) L^{\prime}=B_{1 \rightarrow 2}\left(\tilde{\Phi}_{1}\right) \tilde{L}, \tilde{l}_{1}=\mathbb{B}_{1 \rightarrow 1}^{i}\left(\tilde{\Phi}_{1}\right) \tilde{L} \\
& l_{1}^{i i}=B_{2 \rightarrow 2}^{i i} l_{1}^{\prime i}=B_{1 \rightarrow 2}\left(\tilde{\Phi}_{1}^{-1}\right) \tilde{l}_{1}, \tilde{L}_{1}=B_{1 \rightarrow 1}^{i i}\left(\tilde{\Phi}_{1}^{-1}\right) \tilde{l}_{1}=B_{1 \rightarrow 1}\left(\tilde{\Phi}_{1}\right) \tilde{L} \\
& L_{1}^{\prime}=B_{2 \rightarrow 2}^{i i i}\left(\Phi_{1}^{\prime-1} x^{\prime}\right) l_{1}^{\prime i i}=B_{2 \rightarrow 2}\left(\Phi_{1}^{\prime}\right) L^{\prime}=B_{1 \rightarrow 2}\left(\tilde{\Psi}_{1}\right) \tilde{L}_{1} \\
& \quad l_{12}^{i}=B_{2 \rightarrow 2}^{i}\left(\Phi_{12}^{\prime}\right) L_{1}^{\prime}=\mathbb{B}_{1 \rightarrow 2}\left(\tilde{\Phi}_{12}\right) \tilde{L}_{1}
\end{aligned}
$$




$$
\begin{aligned}
& \tilde{l}_{12}=B_{1 \rightarrow 1}^{i}\left(\widetilde{\Phi}_{12}\right) \tilde{L}_{1}, l_{12}^{i i}=B_{2 \rightarrow 2}^{i i} l_{12}^{i}=B_{1 \rightarrow 2}\left(\tilde{\Phi}_{12}^{-1}\right) \tilde{l}_{12}, \\
& \tilde{L}_{12}=B_{1 \rightarrow 1}^{i i}\left(\widetilde{\Phi}_{12}^{-1}\right) \tilde{l}_{12}=B_{1 \rightarrow 1}\left(\tilde{\Phi}_{12}\right) \tilde{L}_{1}, \\
& L_{12}^{\prime}=B_{2 \rightarrow 2}^{i i i}\left(\Phi_{12}^{\prime-1} x_{1}^{\prime}\right) l_{12}^{i i}=B_{2 \rightarrow 2}\left(\Phi_{12}^{\prime}\right) L_{1}^{\prime}=B_{1 \rightarrow 2}\left(\tilde{\Psi}_{12}\right) \tilde{L}_{12},
\end{aligned}
$$

and the remaining operators are given by the exchange of indices $1 \leftrightarrow 2$. The space variables and eigenfunctions can be traced from the original eigenfunctions $\widetilde{\Phi}_{1}, \widetilde{\Phi}_{2}$ and $\widetilde{\Psi}$ for $\widetilde{L}$ using the corresponding transformations on the operators:

$$
\begin{aligned}
& \Phi_{1}^{\prime}=\widetilde{\Phi}_{1}, \tilde{\Psi}_{1}=x_{1}^{\prime}=\widetilde{\Phi}_{1}\left(\widetilde{\Phi}_{1}^{-1} \tilde{\Psi}\right)_{x}, \widetilde{\Phi}_{12}=\widetilde{\Phi}_{1}\left(\widetilde{\Phi}_{1}^{-1} \widetilde{\Phi}_{2}\right)_{x}, \\
& \Phi_{12}^{\prime}=\widetilde{\Phi}_{12}, \quad \widetilde{\Psi}_{12}=x_{12}^{\prime}=\widetilde{\Phi}_{12}\left(\widetilde{\Phi}_{12}^{-1} \widetilde{\Psi}_{1}\right)_{x} .
\end{aligned}
$$

The final Diagram 4 clearly indicates, how intimately the three integrable hierarchies of $\mathrm{KP}$, modified $\mathrm{KP}$ and Dym equations are related. Although a large number of different gauge transformations and reciprocal links were found for these hierarchies, Diagram 4 shows the amazing and simple pattern

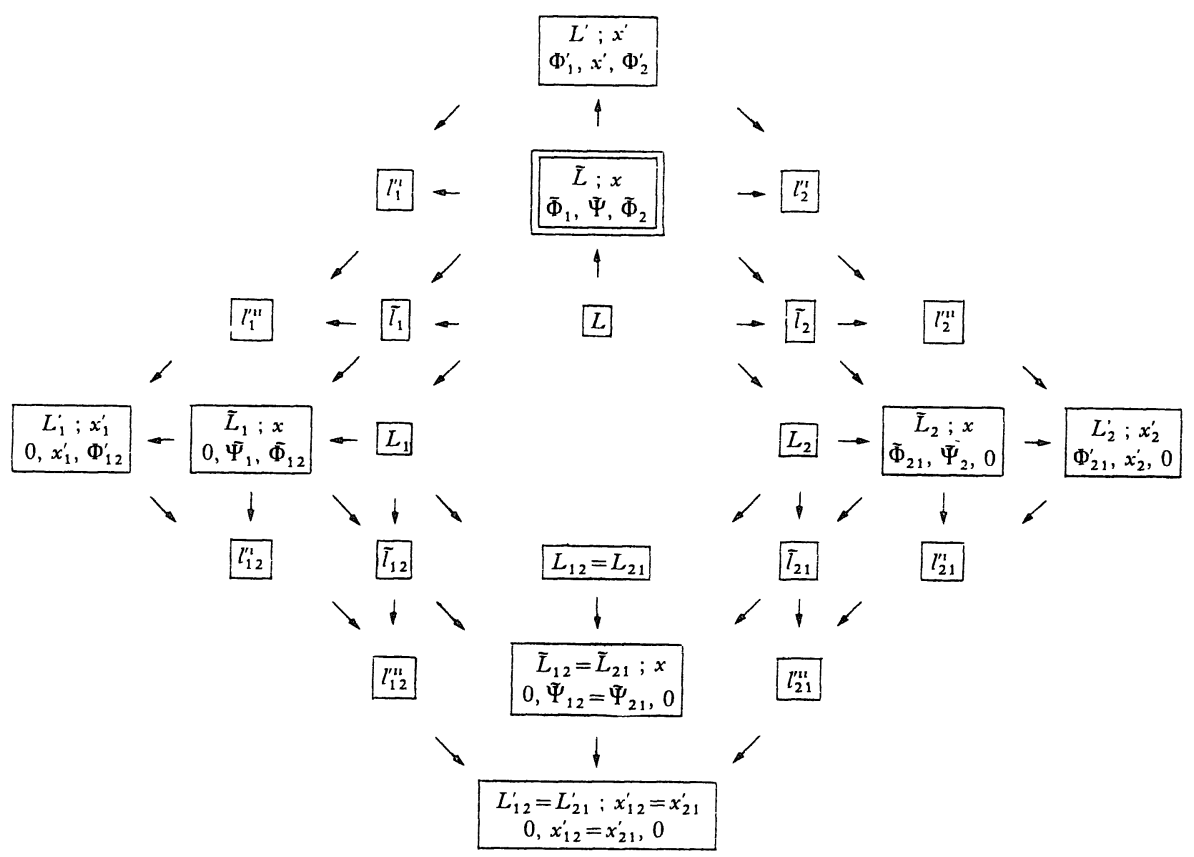

Diagram 4 
formed by these relations. The main transformations are given by the connecting links $\mathbb{B}_{0 \rightarrow 1}$ and $\mathbb{B}_{1 \rightarrow 2}$, which are supplemented by the auto-Bäcklund relations $\mathbb{B}_{0 \rightarrow 0}, \mathbb{B}_{1 \rightarrow 1}$, and $\boldsymbol{B}_{2 \rightarrow 2}$. In addition, it was found that some of these links decompose into a sequence of more elementary transformation, which still form a simple and beautiful pattern according to Diagram 4.

These considerations are applicable to the general hierarchies of $\mathbb{K P}$, modified KP and Dym equations as well as to the $1+1$-dimensional reductions connected with scattering problems of the type (5.3). Hence, Diagram 4 is valid for a large number of integrable hierarchies, some of which were discussed in Section 3. We point out, however, that these general results cannot be applied to all equations of Section 3. In particular, the gauge transformations and reciprocal links discussed here will not leave the Kupershmidt constraints of Section 3.3 invariant. It turns out that for constrained equations further gauge transformations may be considered, which are compatible with such constraints.

\section{$\S 6$. Conclusions}

We have considered three classes of integrable Lax equations on the algebra of pseudo-differential symbols, which are connected to three different Lie algebra decompositions. For the first class this construction is a meanwhile classical approach covering the KP hierarchy as well as $1+1$-dimensional reductions associated with Gelfand-Dikii spectral problems. The extension to the two additional "non-standard" cases is in principle a straightforward generalization of the techniques used in the original $\mathbb{K P}$ context. On a general level, the new systems are identified as the modified $\mathrm{KP}$ hierarchy and the Dym hierarchy. As in the KP case, various reductions of the nonstandard hierarchies lead to a large number of integrable $1+1$-dimensional equations. This approach turns out to be very rich: a surprising variety of the "classical" soliton systems such as the $K \mathrm{dV}$ equation, the modified $\mathrm{KdV}$ equation, the Dym equation and many others is covered by this construction. In addition, many novel integrable equations are discovered on the way.

Using the $r$-matrix approach one has a general scheme to obtain the multi-Hamiltonian formulations for these equations. In fact, it was demonstrated that most of the "classical" bi-Hamiltonian formulations for the well-known equations covered by this approach may be explained via 
the general Poisson brackets associated with $r$-matrices. For the novel equations reveiled in this construction the calculation of the multi-Hamiltonian structure thus has become a straightforward exercise. Nevertheless, certain problems concerning the Hamiltonian approach still seem to be open. Thus, the general quadratic bracket is not a Poisson bracket for the nonstandard cases, but still leads to Hamiltonian formulations using suitable reduction techniques. Further, sometimes certain technical difficulties are faced in the reductions. For instance, Kupershmidt found three local Poisson structures for the Kaup-Broer system which is the simplest realization of the nonstandard class $k=1$. Here, only the first of these formulations is identified with the linear bracket of the corresponding $r$-matrix. The reductions for the higher brackets are technically involved in this case and it remains open, whether Kupershmidt's additional Hamiltonian operators are hidden in the higher brackets.

The maybe most surprising part of this approach is the general and compact picture on (reciprocal) Bäcklund and auto-Bäcklund transformations linking the equations. The classical Darboux transformations connected to the KP class are generalized, a total of ten different transformations was found in Section 5. Surprisingly all these transformations fall into a simple pattern, culminating in the Bianchi Diagram 4. The generality of the underlying theorems gives a unified approach to the Bäcklund transformations for the nonlinear equations covered by the general hierarchies of $\mathrm{KP}$, modified $\mathrm{KP}$ and Dym. Thus, it now is a fairly straightforward exercise to calculate the links for the novel equations reveiled. In particular, using the new Darboux transformations iteratively, it is possible to derive Wronskian solution formulas for the Dym type equations of the class $k=2$, both in $1+1$ as well as in $2+1$ dimensions. This will be investigated in detail elsewhere.

Finally, a straightforward generalization of the present approach is suggested by considering Lax operators of the form $L=\Sigma u_{i}(x) \partial^{i}$, where the $u_{i}$ are not just scalar functions any more but take values in some finite dimensional algebra. The standard decomposition into differential operators versus integration symbols then leads to the multi-component KP hierarchy. It was recently shown ([37]) that further "classical" soliton systems such as the nonlinear Schrödinger equation and the DaveyStewartson equation can be described this way. A corresponding "nonstandard" class of equations is obtained from a modified Lie algebra decomposition, in which the zero order terms of the pseudo-differential symbols are interpreted as part of the integration operators (" $k=1$ "). This 
class encapsulates 2+1-dimensional equations such as the Ishimori hierarchy. The picture on relating gauge transformations as developed here applies to this non-abelian case without major modifications. In this context, the Ishimori hierarchy is identified as the modification of the DaveyStewartson hierarchy in a very natural way. In the non-abelian case it is more convenient to consider the dynamics of dressing operators instead of Lax operators. The Darboux theorems for the Lax operators considered here translate to analogous results for the dressing operators. Details on this approach are discussed in [38]. We finally remark that in this generalization to non-abelian coefficients of the differential symbols the class $k=2$ will not be present, as the underlying Lie algebra decomposition will not work for non-commuting coefficients. However, a rich structure is obtained when additional decompositions are considered which involve the finite dimensional algebra from which the coefficients are chosen.

\section{Acknowledgements}

One of the authors (B.G.K.) gratefully acknowledges support by the Deutsche Forschungsgemeinschaft. He thanks Prof. B. Fuchssteiner for the kind hospitality during his stay at the University of Paderborn, where the main results were established.

\section{References}

[1] Zakharov, V.E., Manakov, S.V., Novikov, S.P. and Pitaevskii, L.P., Theory of Solitons. The inverse scattering method, Nauka, Moscow, 1980; Consultants Bureau, New York, London, 1984.

[2] Ablowitz, M.J. and Segur, H., Solitons and the Inverse Scattering Transform, SIAM, Philadelphia, 1981.

[ 3 ] Newell, A.C., Solitons in Mathematics and Physics, SIAM, Philadelphia, 1985.

[4] Fadeev, L.D. and Takhtajan, L.A., Hamiltonian Methods in the Theory of Solitons, Springer, Berlin, 1987.

[ 5 ] Gelfand, I.M. and Dikii, L.A., Fractional powers of operators and Hamiltonian systems, Funct. Anal. Appl., 10 (1976), 259-273.

[ 6 ] Manin, Y.I., Algebraic aspects of nonlinear differential equations, J. Soviet Math., $\mathbb{1}$ (1979), 1-122.

[ 7 ] Adler, M., On a trace functional for pseudo differential operators and the symplectic structure of the Korteweg-de Vries equation, Invent. Math., 50 (1979), 219-248.

[ 8 ] Drinfeld, V.G. and Sokolov, V.V., Equations of Korteweg-de Vries type and simple Lie algebras, Dokl. Akad. Nauk. SSSR, 258 (1981), 11-16.

[9] Kupershmidt, B.A. and Wilson, G., Modifying Lax equations and the second Hamiltonian structure, Invent. Math., 62 (1981), 403-436.

[10] Sato, M., Soliton equations as dynamical systems on infinite Grassmann manifold, RIMS Kokyuroku, Kyoto Univ., 439 (1981), 30-46. 
[11] Sato, M. and Sato, Y., Soliton equations as dynamical systems on infinite Grassmann manifold, in Nonlinear Partial Differential Equations in Applied Science, pp. 259-271, H. Fujita, P.D. Lax and G. Strang (eds.), Kinokuniya/North Holland, Tokyo, 1983.

[12] Date, E., Jimbo, M., Kashiwara, M. and Miwa, T., Transformation groups for soliton equations, in Nonlinear Integrable Systems-Classical Theory and Quantum Theory, pp. 39-119, M. Jimbo and T. Miwa (eds.), World Scientific, Singapore, 1983.

[13] Jimbo, M. and Miwa, T., Solitons and infinite-dimensional Lie algebras, Publ. RIMS, Kyoto Univ., 19 (1983), 943-1001.

[14] Ohta, Y., Satsuma, J., Takahashi, D. and Tokihiro, T., An elementary introduction to Sato theory, Progr. Theor. Phys. Suppl., 94 (1988), 210-241.

[15] Drinfeld, V.G., Hamiltonian structures on Lie groups, Lie bialgebras and the geometric meaning of classical Yang Baxter equations, Soviet Math. Dokl., 27 (1983), 68-70.

[16] Semenov-Tian-Shansky, M.A., What is a classical $r$-matrix?, Funct. Anal. Appl., 17 (1983), 259-272.

[17] —, Dressing transformations and Poisson group actions, Publ. RIMS, Kyoto Univ., 21 (1985), 1237-1260.

[18] Li, L.C. and Parmentier, S., Nonlinear Poisson structures and $r$-matrices, Comm. Math. Phys., 125 (1989), 545-563.

[19] Oevel, W. and Ragnisco, O., R-matrices and higher Poisson brackets for integrable systems, Physica A, 161 (1990), 181-220.

[20] Reiman, A.G., Integrable Hamiltonian systems connected with graded Lie algebras, $J$. Soviet Math., 19 (1982), 1507-1545.

[21] Kupershmidt, B.A., Mathematics of dispersive water waves, Comm. Math. Phys., 99 (1985), 51-73.

[22] Kiso, K., A remark on the commuting flows defined by Lax equations, Progr. Theor. Phys., 83 (1990), 1108-1114.

[23] Konopelchenko, B.G. and Dubrovsky, V.G., Some new integrable nonlinear evolution equations in 2+1 dimensions, Phys. Lett. A, 102 (1984), 15-17.

[24] Oevel, W. and Rogers, C., Gauge Transformations and Reciprocal Links in $2+1$ Dimensions, Rev. in Math. Phys., 5 (1993), 299-330.

[25] Fordy, A.P.F. and Gibbons, J., Some remarkable nonlinear transformations, Phys. Lett. $A, 75$ (1980), 325.

[26] Sawada, K. and Kotera, T., A method for finding $N$-soliton solutions of the K.d.V. equation and K.d.V.-like equation, Progr. Theor. Phys., 51 (1974), 1355-1367.

[27] Belavin, A.A. and Drinfeld, V.G., Solutions of the classical Yang-Baxter equation for simple Lie algebras, Funct. Anal. Appl., 16 (1982), 159-183.

[28] —- Triangle equations and simple Lie algebras, in Math. Phys. Rev. 4 (Soviet Scientific Reviews, Section C), S.P. Novikov (ed.), Harwood Academic Publishers, London, 1987.

[29] Fuchssteiner, B. and Fokas, A.S., Symplectic structures, their Bäcklund transformations and hereditary symmetries, Physica D, 4 (1981), 47-66.

[30] Weiss, J., Tabor, M. and Carnevale, G., The Painlevé property for partial differential equations, J. Math. Phys., 24 (1983), 522-526.

[31] Weiss, J., The Painlevé property for partial differential equations. II. Bäcklund transformation, Lax pairs, and the Schwarzian derivative, J. Math. Phys., 24 (1983), 1405-1413.

[32] Char, B.W., Geddes, K.O., Gonnet, G.H. and Watt, S.M., Maple User's Guide, Watcom Publication, Waterloo, 1985.

[33] Kawamoto, S., An exact transformation from the Harry Dym equation to the modified KdV equation, J. Phys. Soc. Japan, 54 (1985), 2055-2056.

[34] Konopelchenko, B.G., On the gauge invariant description of the evolution equations integrable by Gelfand-Dikij spectral problems, Phys. Lett. A, 92 (1982), 323-327.

[35] Rogers, C., The Harry Dym equation in $2+1$ dimensions: a reciprocal link with the 
Kadomtsev-Petviashvili equation, Phys. Lett. A, 120 (1987), 15-18.

[36] Rogers, C. and Wong, P., On reciprocal Bäcklund transformations of inverse scattering schemes, Phys. Scripta, 30 (1984), 10-14.

[37] Kajiwara, K., Matsukidaira, J. and Satsuma, J., Conserved quantities of the two-component KP hierarchy, Phys. Lett. A, 146 (1990), 115-118.

[38] Konopelchenko, B.G. and Oevel, W., Matrix Sato theory and integrable equations in 2+1 dimensions, in Nonlinear Evolution Equations and Dynamical Systems '91, Boiti, M., Martina, L. and Pempinelli F. (eds), World Scientific, Singapore, (1992), 87-96.

Note added in Proof. The three integrable hierarchies of KP, modified KP and Dym equations as well as their relations were also considered in Aratyn, H., Nissimov, E., Pacheva, $\mathrm{S}$. and Vaysburd, I., $R$-Matrix Formulation of the KP Hierarchies and Their Gauge Equivalence, Phys. Lett. B, 294 (1992), 167-176. 